\title{
Foliated Positive Scalar Curvature
}

\author{
Dissertation \\ zur Erlangung des mathematisch-naturwissenschaftlichen Doktorgrades \\ „Doctor rerum naturalium“ \\ der Georg-August-Universität Göttingen \\ im Promotionsprogramm Mathematische Wissenschaften \\ der Georg-August University School of Science (GAUSS) \\ vorgelegt von \\ Jialong DENG \\ aus Guangdong, China
}

Göttingen, 2021 
Betreuungsausschuss

Prof. Dr. Thomas Schick (Mathematisches Institut)

Prof. Dr. Viktor PIDSTRYGaCh (Mathematisches Institut)

Mitglieder der Prüfungskommission

Referent: Prof. Dr. Thomas ScHICK

Korreferent: Prof. Dr. Viktor PidsTRYGaCH

Weitere Mitglieder der Prüfungskommission:

Prof. Dr. Preda MinaILEscu (Mathematisches Institut)

Prof. Dr.Ulrich STUHLER (Mathematisches Institut)

Prof. Dr.Christoph LeHrenfeld (Institut für Num. und Angew. Mathematik)

Prof. Dr. Max WardeTzKy (Institut für Num. und Angew. Mathematik)

Tag der mündlichen Prüfung: Oktober 7, 2021 


\section{Abstract}

In this thesis we study different questions on scalar curvatures. The first part is devoted to obstructions against existence of a (Riemannian) metric with positive scalar curvature on a closed manifold. The second part investigates the synthetic definition of scalar curvature bounded below on metric measure spaces. In the third and fourth part, we define and study weighted scalar curvature on a smooth metric measure space. We show rigidity results about scalar curvature bounded below and a sphere theorem for $\operatorname{RCD}(n-1, n)$ spaces in the final part. 



\section{Acknowledgments}

I would like to first extend my deep gratitude to Prof. Dr. Thomas Schick, who always gave me abundant freedom and flexibility while guided me to be an independent scholar. My thanks also go to Dr. Simone Cecchini and other reviewers for helping me to improve the manuscript.

I am extremely grateful to my family and friends for their constant support throughout the course of my growth.

The funding comes from China Scholarship Council (during October 2017 to March 2021) and the doctoral scholarship of Mathematisches Institut, Georg-August-Universität, Göttingen (during April 2021 to September 2021). 



\section{Contents}

Abstract iii

\begin{tabular}{ll}
\hline Acknowledgments & v
\end{tabular}

\begin{tabular}{lll}
\hline 1 & Introduction & 1
\end{tabular}

$\begin{array}{|ll|}2 & \text { Enlargeable Length-structures and Scalar Curvatures }\end{array}$

$2.1 \quad$ Introduction $\ldots \ldots \ldots \ldots \ldots \ldots \ldots$

2.2 Enlargeable length-structures, PSC-metrics and locally CAT(0)-metrics . 16

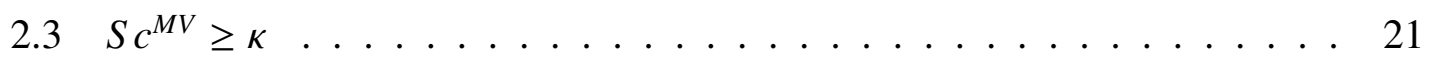

3 Curvature-Dimension Condition Meets Gromov's $n$-Volumic Scalar Curvature 31

3.1 CD meets $n$-volumic scalar curvature . . . . . . . . . . . . . . . 32

3.2 smGH-Convergence . . . . . . . . . . . . . . . . . 41

$\begin{array}{|ll|}4 & \text { Weighted Scalar Curvature } S c_{\alpha, \beta}\end{array}$

$4.1 \quad$ Spin manifold and $S c_{\alpha, \beta}>0 \ldots \ldots \ldots \ldots \ldots$

4.2 Conformal to PSC-metrics . . . . . . . . . . . . . . 51

$4.3 \quad f$-minimal hypersurface and $S c_{\alpha, \beta}>0 \ldots \ldots \ldots \ldots$

4.4 Weighted rigidity $\ldots \ldots \ldots \ldots \ldots \ldots \ldots$

vii 
5 Weighted Scalar Curvature in Four Dimensions 63

5.1 Seiberg-Witten invariants and weighted scalar curvature . . . . . . . . . 63

5.2 General relativity on metric measure spaces . . . . . . . . . . . . 67

$6 \quad$ Sphere Theorems with and without Smoothing 69

6.1 Introduction . . . . . . . . . . . . . . . . . . . . . 69

6.2 Harmonic maps with condition C . . . . . . . . . . . . . . 70

6.3 Non-collapsed RCD spaces and mean distance . . . . . . . . . . . . 76 


\section{Introduction}

Consideration of how the (flexible) topology influences the (possible) geometry of the space started with the Gauss-Bonnet theorem and the influential work of Hopf in the 1930s. Given a smooth Riemannian manifold $\left(M^{n}, g\right)$, the volume of the small ball $B_{r}(x) \subset M$ is

$$
\operatorname{vol}_{\mathrm{g}}\left(B_{r}(x)\right)=\operatorname{vol}_{E}\left(B_{r}\right)\left[1-\frac{S c_{g}}{6(n+2)} r^{2}+O\left(r^{4}\right)\right]
$$

as $r \rightarrow 0$, where $\operatorname{vol}_{E}\left(B_{r}\right)$ is the volume of the ball of radius $r$ in the Euclidean space $\mathbb{R}^{n}$ and $S c_{g}$ is the scalar curvature of $\left(M^{n}, g\right)$.

A smooth function which is negative somewhere on a smooth closed $n$-manifold $(n \geq 3)$ can be realized as the scalar curvature of a Riemanian metric according to the Kazdan-Warner theorem [KW75]. If the closed manifold admits a Riemannian metric with non-negative (but not identically zero) scalar curvature, then we can deform the metric to a new Riemannian metric with positive scalar curvature using the result of Ricci-flow arguments. Thus, it is natural to ask the following question:

Which manifold may admit a metric with positive scalar curvature (PSC-metric)?

Combining Schoen-Yau's results on the structure of 3-manifolds with positive scalar curvature and [SY79a] Thurston-Perleman's Geometrization Theorem shows that closed orientable 3-manifolds $M$ admitting PSC-metrics must be spherical 3-manifolds, $S^{2} \times S^{1}$, or the connected sum of spherical 3-manifolds and $\sum S^{2} \times S^{1}$.

In four dimensions, Seiberg-Witten invariants can be used as a tool whether the manifolds admit a PSC-metric. 
In general, there are two fundamental approaches to the question. One is Schoen-Yau's minimal surface method [SY79b]. The other one is based on index theorical technique which is invented by Lichnerowicz [Lic63] and is developed by Hitchin [Hit74], GromovLawson [GL83], Rosenberg [Ros83] and many others. In particular, Hitchin refined Lichnerowicz's result to show that a spin manifold $M^{n}$ with a PSC-metric implies the $\alpha$-invariant $\alpha\left(M^{n}\right)$ living in the KO-group vanishes.

The question was settled by Stolz [Sto92] for closed smooth simply connected manifolds of dimensions $\geq 5$.

Theorem 1.1. For simply connected smooth $n$-manifold $M^{n}(n \geq 5)$,

- [Gromov-Lawson 1980] if $M^{n}$ is not spin, then it carries PSC-metrics;

- [Stolz 1990] if $M^{n}$ is spin, then it carries PSC-metrics if and only if $\alpha\left(M^{n}\right)=0$.

There are several survey articles about the scalar curvature of a Riemannian metric, for example, [Ros07], [Sch14] and [Gro19]. One can find more previous results in those papers.

The first part of this thesis investigates the obstructions to the existence of a PSC-metric on a closed non-simply connected smooth manifold. In particular, this relates to the open conjecture whether a closed aspherical manifold cannot admit a PSC-metric.

Based on index theory methods, Gromov and Lawson introduced enlargeability as an obstruction based on index theory in [GL80]. Later, they relaxed the spin assumption in dimensions less than 8 in [GL83, section 12]. Recently, Schoen and Yau showed that the manifold $N^{n} \# T^{n}$ carries no PSC-metrics by geometric measure theory and minimal surfaces methods in [SY17], where $N^{n}$ is any closed oriented smooth $n$-manifold and $T^{n}$ is a torus. Then, Cecchini and Schick used Schoen and Yau's results to show that a closed enlargeable manifold cannot carry any PSC-metric in [CS21].

Both enlargeabilities mentioned above are defined on Riemannian metrics and need at least $C^{1}$-smoothness for the maps. Here an enlargeable length-structure will be defined for 
length metric spaces and the maps only require to be continuous. A metric space $(X, d)$ is a length metric space if the distance between each pair of points equals the infimum of the lengths of curves joining the points.

We call that a topological manifold $X$ endowed with a complete length metric is $\varepsilon$ hyperspherical if it admits a continuous map $f$ to $S^{n}(n=\operatorname{dim}(X))$ which is constant at infinity, of non-zero degree and such that

$$
\operatorname{Lip}(f):=\sup _{\substack{a \neq b \\ a, b \in X}} \frac{d_{S^{n}}(f(a), f(b))}{d_{X}(a, b)}<\varepsilon .
$$

Here constant at infinity means that there is a compact subset such that $f$ maps the complement of the compact subset to a point in $S^{n}$ and $S^{n}$ is endowed with standard round metric. $S^{n}$ is also a length metric space induced by the standard round metric $d_{S^{n}}$.

Definition 1.2 (Enlargeable length-structures [Den21b]). A length metric $d$ on a closed orientable n-dimensional topological manifold $X^{n}$ is said to be enlargeable if for each $\varepsilon>0$ there is an oriented covering manifold $\widetilde{X^{n}}$ endowed with the induced metric $\widetilde{d}$ that is $\varepsilon$-hyperspherical.

An enlargeable length-structure on $X^{n}$ is a BiLipshitz equivalent class of an enlargeable metric.

An enlargeable Riemannian length-structure on a closed orientable smooth manifold is an enlargeable length-structure that contains a length metric induced by a Riemannian metric on the manifold.

The length metric induced by a Riemannian flat metric on $T^{n}$ is an enlargeable metric and $T^{n}$ endowed with this enlargeable length-structure is an important example of the manifolds with an enlargeable length-structure.

Theorem 1.3. [Den21b] Let $X^{n}(2 \leq n \leq 8)$ be a closed orientable smooth manifold, then $X^{n}$ carries no PSC-metrics in its enlargeable length-structures. 
Davis, Januszkiewicz and Lafont [DJL12] constructed a closed smooth four-dimensional manifold $M^{4}$, which supports locally CAT(0)-metrics and whose universal cover $\widetilde{M^{4}}$ is diffeomorphic to $\mathbf{R}^{4}$, but $\pi_{1}$ is not isomorphic to the fundamental group of any compact Riemannian manifold with non-positive curvature.

Our enlargeable length-structures can be used to detected new manifolds (comparing with Gromov-Lawson's method) which carries not PSC-metrics. For example, the locally CAT(0)-metrics which were constructed by Davis-Januszkiewicz-Lafont above are strongly equivalent to a Riemannian metric on the exotic aspherical 4-manifolds. Therefore, the connected sum of a closed 4-manifold and a Davis-Januszkiewicz-Lafont's exotic aspherical 4-manifold, as new examples detected by our methods, carries no PSC-metrics.

Assuming that the manifold has a PSC-metric, one may ask the question whether the property of scalar curvature bounded below is preserved by taking some kind of limit of a family of Riemannian manifolds, i.e. the stability of Riemannian manifolds with scalar curvature bounded below. The question was inspired by the following facts.

The concept of lower bounded curvature on the metric space and its stability originated with Alexandrov in the 1950s and has been studied extensively by Burago, Cheeger, Fukaya, Gromov, Perelman and others. Furthermore, the properties of the limit of Riemannian manifolds with sectional curvature bounded below were essentially used in Pereleman's proof of the Poincare conjecture [Per02]. Riemannian manifolds with Ricci curvature bounded below were generalized to metric measure spaces and the question of stability was mainly solved by Sturm [Stu06a] and Lott-Villani [LV09] independently. The structure of Riemannian manifolds with Ricci curvature bounded below was explored by CheegerColding [CC97] in a series of articles and then it was used essentially in Chen-DonaldsonSun's proof of the Donaldson-Tian-Yau conjecture [CDS15].

Since the limit of Riemannian manifolds with scalar curvature bounded below may not be smooth or even a manifold, Gromov proposed a synthetic treatment of scalar curvature bounded below, which is called the $n$-volumic scalar curvature bounded below 
$\left(S c^{\mathrm{vol}_{n}} \geq \kappa\right)$ Gro17]. Roughly, this means the measure is locally volume-wise smaller than the model space and the idea is similar to the definition using comparison triangle in the Alexandrov geometry. Furthermore, the $n$-dimensional Alexandrov space with curvature $\geq \kappa$ equipped with the volume measure satisfies Lott-Villani-Sturm's weak curvaturedimension condition for dimension $n$ and curvature $(n-1) \kappa$, i.e. $C D(\kappa, n)$-condition, as shown by Petrunin [ [Pet11] and Zhu-Zhang [ZZ10] .

The second part of this thesis proposes a modified definition of $n$-volumic scalar curvature bounded below $\left(S c^{\mathrm{vol}_{n}} \geq \kappa\right)$ and adds the $n$-dimensional condition to it.

Definition 1.4 ( $n$-dimensional condition [Den21a]). For given positive natural number $n$, the mm-space $X=(X, d, \mu)$ satisfies the $n$-dimensional condition if

$$
\lim _{r \rightarrow 0} \frac{\mu\left(B_{r}(x)\right)}{\operatorname{vol}_{E}\left(B_{r}\left(\mathbf{R}^{n}\right)\right)}=1
$$

for every $x \in X$, where $B_{r}\left(\mathbf{R}^{n}\right)$ is the closed $r$-ball in the Euclidean space $\mathbf{R}^{n}$ and the $B_{r}(x)$ is the closed $r$-ball with the center $x \in X$.

Then we combine the curvature-dimension condition $C D(\kappa, n)$ with the $n$-dimensional condition to show the following theorem.

Theorem 1.5 ([Den21a $])$. Assume that the metric measure space $\left(X^{n}, d, \mu\right)$ satisfies $n$ dimensional condition and the curvature-dimension condition $C D(\kappa, n)$ for $\kappa \geq 0$ and $n \geq 2$, then $\left(X^{n}, d, \mu\right)$ satisfies $S c^{\mathrm{vol}_{n}}\left(X^{n}\right) \geq n \kappa$.

Since only the $n$-dimensional condition and the generalized Bishop-Gromov volume growth inequality are needed in the proof, the curvature-dimension condition can be replaced with Ambrosio-Gigli-Savare's Riemannian curvature condition $R C D(\kappa, n)$ [AGS14] or with Ohta's measure concentration property $\operatorname{MCP}(\kappa, n)$ [Oht07].

As the metric measure spaces with $S c^{\mathrm{vol}_{n}} \geq \kappa \geq 0$ may not be stable under measured Gromov-Hausdorff convergence, we define the strongly measured Gromov-Hausdorff 
convergence on metric measure spaces, which requires the measures to converge strongly. We also define the scalar curvature radius (SC-radius) on the metric measure spaces. Under this premise, we prove the following:

Theorem 1.6 (Stability [Den21a] $)$. If compact metric measure spaces $\left(X_{i}^{n}, d_{i}, \mu_{i}\right)$ with $S c^{\mathrm{vol}_{n}}\left(X_{i}^{n}\right) \geq \kappa \geq 0, S C$-radius $\geq R>0$, and $\left(X_{i}^{n}, d_{i}, \mu_{i}\right)$ strongly measured GromovHausdorff converge to the compact metric measure space $\left(X^{n}, d, \mu\right)$ with n-dimensional condition, then $X^{n}$ also satisfies $S c^{\mathrm{vol}_{n}}\left(X^{n}\right) \geq \kappa$ and the $S C$-radius $r_{X^{n}} \geq R$.

One potential application of stability results about scalar curvature is in general relativity as predicted by the Sormani-Wenger's intrinsic flat convergence [LS14].

It is well-known that Gigli's infinitesimally Hilbertian property [Gig15] of metric measure space can be seen as a Riemannian condition in $R C D(\kappa, n)$ space. The infinitesimally Hilbertian property can also be used as a Riemannian condition in the metric measure spaces with $S c^{\mathrm{vol}_{n}} \geq 0$, so that we can restrict it to more regular spaces.

Definition 1.7 ([Den21a $])$. The compact metric measure space $(X, d, \mu)$ is a Riemannian $n$-volumic scalar curvature $\geq \kappa$ space $(\operatorname{RSC}(\kappa, n)$ space) if it is infinitesimally Hilbertian and satisfies the $S c^{\operatorname{vol}_{n}}(X) \geq \kappa \geq 0$.

Note that a $n$-dimensional Alexandrov spaces $A l^{n}(\kappa)$ with curvature bounded below by $\kappa$ is infinitesimally Hilbertian. Therefore, we have

$$
A l^{n}(\kappa) \Rightarrow R C D((n-1) \kappa, n) \Rightarrow R S C((n(n-1) \kappa, n)
$$

on $\left(X^{n}, d, \mathcal{H}^{n}\right)$, where the measure $\mathcal{H}^{n}$ is the $n$-dimensional Hausdorff measure that satisfies the $n$-dimensional condition.

Motivated by the importance of Ricci Bakry-Emery curvature $\geq \kappa[$ BE85], that is

$$
\operatorname{Ric}_{f}^{M}=\operatorname{Ricc}+\operatorname{Hess}(f) \geq \kappa
$$


on the smooth metric measure space $\left(M^{n}, g, e^{-f} d \mathrm{vol}_{g}\right.$ ) (also known as weighted Riemannian manifolds in some references), where $f$ is the $C^{2}$-function on $M^{n}, g$ is a $C^{2}$-Riemannian metric and $n \geq 2$, it is natural to ask what is the right way to define the scalar curvature on the smooth metric measure space.

In the third part of this thesis, we define and study the scalar curvature $S c_{\alpha, \beta}$ on smooth metric measure spaces by

$$
S c_{\alpha, \beta}:=S c_{g}+\alpha \Delta_{g} f-\beta\left\|\nabla_{g} f\right\|_{g}^{2}
$$

for the smooth metric measure space $\left(M^{n}, g, e^{-f} d \operatorname{vol}_{g}\right)$. Note that here the Laplacian $\triangle_{g}$ is the trace of the Hessian.

If an orientable closed surface with density $\left(\Sigma, g, e^{-f} d \operatorname{vol}_{g}\right)$ satisfies $S c_{\alpha, \beta}>0$ and $\beta \geq 0$, then $\Sigma$ is a 2 -sphere, generalizing the classical Gauss-Bonnect result. We investigate similar consequence of $S c_{\alpha, \beta}>0$ in higher dimensions in the following theorem.

Theorem 1.8 ([Den21a $])$. Let $\left(M^{n}, g, e^{-f} d \operatorname{Vol}_{g}\right)$ be the closed smooth metric measure space with $S c_{\alpha, \beta}>0$, then we have the following conclusions:

1. If $M^{n}$ is spin manifold, $\alpha \in \mathbb{R}$, and $\beta \geq \frac{|\alpha|^{2}}{4}$, then all harmonic spinors of $M^{n}$ vanishes. In particular, the $\widehat{A}$-genus and the Rosenberg index of $M^{n}$ vanish.

2. If the dimension $n \geq 3, \alpha \in \mathbb{R}$, and $\beta \geq \frac{(n-2)|\alpha|^{2}}{4(n-1)}$, then there is a metric $\tilde{g}$ conformal to $g$ with positive scalar curvature.

3. If the dimension $n \geq 3, \alpha=2, \beta \geq \frac{n-2}{n-1}$, and $\left(N^{n-1}, \bar{g}\right)$ is a compact $L_{f}$-stable minimal hypersurface of $\left(M^{n}, g, e^{-f} d \mathrm{Vol}_{g}\right)$, then there exists a PSC-metric conformal to $\bar{g}$ on $N^{n-1}$, where $\bar{g}$ is the induced metric of $g$ on $N^{n-1}$.

4. Assume $M^{n}$ is a spin manifold and there exists a smooth non-zero degree 1-contracting map $h:\left(M^{n}, g\right) \rightarrow\left(S^{n}, g_{s t}\right)$. If $\alpha \in \mathbb{R}, \beta \geq \frac{|\alpha|^{2}}{4}$ and $S c_{\alpha, \beta} \geq n(n-1)$, then $h$ is an isometry between the metrics $g$ and $g_{s t}$. 
In the fourth part of this thesis, we study the weighted scalar curvature in four dimensions and show that if the dimension $n=4, \alpha \in \mathbb{R}$, and $\beta \geq \frac{|\alpha|^{2}}{6}$, then the Seiberg-Witten invariants vanish.

In the fifth part of this thesis, we show a new rigidity result for Riemannian manifolds with scalar curvature bounded below. Chern-Goldberg [CG75] defined Condition C for a harmonic map between Riemannian manifolds. We use the harmonic map with Condition $\mathrm{C}$ to prove the following theorem.

Theorem 1.9 ([Den21] $)$. Let $(M, g)$ be an orientable closed Riemannian n-manifold with scalar curvature $\geq n(n-1)$. Suppose there exists a $\left(1, \wedge^{1}\right)$-contracting map $f: M \rightarrow S^{n}$ of non-zero degree and that the map $f$ is harmonic with condition $C \leq 0$, then $f$ is an isometric map.

Inspired by the rigidity result, we also give a new sphere theorem about $\operatorname{RCD}(n-1, n)$ spaces in the fifth part of this thesis. The framework of Riemannian manifolds with Ricci curvature bounded below is generalized to metric measure spaces $(X, d, \mu)$ satisfying Riemannian curvature-dimension condition.

We combine this with bounds on mean distance. Mean distance is an old and wellstudied metric invariant in Graph theory, see [DG77]. Note that mean distance is also called distance covariance in Probability, see [Lyo13].

Theorem 1.10 ([Den21] $)$. Let $\left(X, d, \mathcal{H}^{n}\right)$ be a compact non-collapsed $\operatorname{RCD}(n-1, n)$ space with full support and $\operatorname{md}(X)$ be the mean distance of $\left(X, d, \mathcal{H}^{n}\right)$, then

I. $\operatorname{md}(X) \leq \frac{\pi}{2}$ and $\operatorname{md}(X)=\frac{\pi}{2}$ if and only if $X$ is isometric to the standard round $n$-sphere $S^{n}$ and $\mathcal{H}^{n}=a \mathrm{dVol}$ for some $a$, where $\mathrm{dVol}$ is the volume form of $S^{n}$.

II. there is an $\epsilon(n)>0$ such that $\operatorname{md}(X) \geq \frac{\pi}{2}-\epsilon(n)$ implies that $X$ is homeomorphic to $S^{n}$.

We use Ketterer's maximal diameter theorem [Ket15, Theorem 1.4] and HondaMondello's topological sphere theorem [HM19, Theorem A] (for metric measure spaces) 
to prove the result. We also define a whole family of variants of the concept of mean distance for metric measure spaces and prove sphere theorem for all of them. 



\section{Enlargeable Length-structures and Scalar Curvatures}

In this chapter, we define enlargeable length-structures on closed topological manifolds and then show that the connected sum of a closed $n$-manifold with an enlargeable Riemannian length-structure with an arbitrary closed smooth manifold carries no Riemannian metrics with positive scalar curvature.

We show that closed smooth manifolds with a locally CAT(0)-metric which is strongly equivalent to a Riemannian metric are examples of closed manifolds with an enlargeable Riemannian length-structure. Moreover, the result is correct in arbitrary dimensions based on the main result of a recent paper by Schoen and Yau [SY17].

We also define the positive $M V$-scalar curvature on closed orientable topological manifolds and show the compactly enlargeable length-structures are the obstructions of its existence.

This chapter comes from my published paper [Den21b].

\subsection{Introduction}

The search for obstructions to the existence of a Riemannian metric with positive scalar curvature (PSC-metric) on a closed non-simply connected smooth manifold is an ongoing project. Based on index theory methods, Gromov and Lawson introduced enlargeability as 
an obstruction based on index theory in [GL80]. Later, they relaxed the spin assumption in dimensions less than 8 in [GL83, section 12]. Recently, Schoen and Yau showed that the manifold $N^{n} \# T^{n}$ carries no PSC-metrics by geometric measure theory and minimal surfaces methods in [SY17], where $N^{n}$ is any closed oriented smooth $n$-manifold and $T^{n}$ is a torus. Then, Cecchini and Schick used Schoen and Yau's results to show that a closed enlargeable manifold cannot carry any PSC-metric in [CS21].

Both enlargeabilities mentioned above are defined on Riemannian metrics and need at least $C^{1}$-smoothness for the maps. Here an enlargeable length-structure will be defined for length metric spaces and the maps only require to be continuous. Combining enlargeable length-structures with Gromov's Spherical Lipschitz Bound Theorem (SLB Theorem) [Gro18], a new obstruction to the existence of PSC-metrics and positive $M V$-scalar curvature $S c^{M V}$ on a closed manifold is given. Details will be given later.

Theorem 2.1. Let $X^{n}(2 \leq n \leq 8)$ be a closed orientable smooth manifold, then $X^{n}$ carries no PSC-metrics in its enlargeable length-structures.

Theorem 2.2. Let $N^{n}(2 \leq n \leq 8)$ be an arbitrary closed oriented smooth n-manifold and $M^{n}$ be a closed manifold with an enlargeable Riemannian length-structure. Then $N^{n} \# M^{n}$ does not admit a PSC-metric.

Remark 2.3. In fact, $N^{n} \# M^{n}$ does not admit a complete uniformly PSC-metric for any oriented manifold $N^{n}(2 \leq n \leq 8)$.

Theorem 2.4. If $\left(X^{n}, d, \mathcal{H}_{d}^{n}\right)(n \leq 8)$ satisfies $S c^{M V}\left(X^{n}\right) \geq \kappa>0$, then $d$ is not in any compactly enlargeable length-structures on the closed oriented topological manifold X.

Based on Schoen and Yau's argument [SY17], Gromov proved the SLB Theorem [Gro18, Section 3] for any dimensions. Similarly, another consequence of [SY17] is that theorems A and B are also true in higher dimensions. 
Theorem 2.5. If $N^{n}$ is an arbitrary closed oriented smooth $n$-manifold and $X^{n}$ is a closed manifold with an enlargeable Riemannian length-structure, then $N^{n} \# X^{n}$ carries no PSCmetrics.

In particular, if $M^{n}$ is a closed manifold with an enlargeable Riemannian lengthstructure containing a locally CAT(O)-metric, then $N^{n} \# M^{n}$ does not admit a PSC-metric.

Remark 2.6. Gromov also stated the result which said that a closed spin manifold with a locally CAT(0)-metric carries no PSC-metrics without proof in [Gro19, Section 4.1.2]. Theorem B' supports Gromov's assertion.

Remark 2.7. Though Theorem 2.5 is similar to Cecchini and Schick's Theorem A [CS21] and the starting point of the proofs is Schoen and Yau's new results [SY17], the techniques of the proofs are different. Cecchini and Schick's proof used the standard constructions from geometric measure theory, especially, no symmetrization and no manifolds with boundary, whereas the proof of Theorem 2.5 is an application of Gromov's Spherical Lipschitz Bound Theorem that uses these two ingredients.

Theorem 2.5 is also inspired by an open conjecture that a closed aspherical manifold does not admit a PSC-metric. Kasparov and Skandalis [KS03] used KK-theory to prove the strong Novikov conjecture for CAT(0)-group, implying that any locally CAT(0)-manifold carries no PSC-metrics.

The fact that a closed Riemannian manifold with non-positive sectional curvature is enlargeable is well-known since Gromov and Lawson first proposed the definition of enlargeable manifolds [GL80]. Riemannian metrics with non-positive sectional curvature (non-positive curvature metric) are locally CAT(0)-metrics. If a manifold of dimension 2 or 3 admits a locally CAT(0)-metric, then it also admits non-positive curvature metrics, according to the classic surface theory and the Thurston-Perelmann Geometrization Theorem [DJL12, Proposition 1].

But there is a difference between locally $\mathrm{CAT}(0)$-metrics and non-positive curvature 
metrics in dimensions $\geq 4$. Aravinda and Farrell [AF94] showed that the existence of nonpositive curvature metric is not a homeomorphism invariant in general, but the existence of a locally CAT(0)-metric is homeomorphism invariant. The existence of a non-positive curvature metric depends on that of the smooth structure. Furthermore, locally CAT(-1)manifolds which do not support a smooth structure in dimensions 8 were constructed in [DJL12, Section 3.1].

Aspherical $n$-manifolds $(n \geq 4)$ that are not covered by Euclidean space were first constructed by Davis [Dav83]. Davis, Januszkiewicz and Lafont [DJL12] constructed a closed smooth four-dimensional manifold $M^{4}$, which supports locally CAT(0)-metrics and whose universal cover $\widetilde{M^{4}}$ is diffeomorphic to $\mathbb{R}^{4}$, but $\pi_{1}$ is not isomorphic to the fundamental group of any compact Riemannian manifold with non-positive curvature. In dimensions $\geq$ 5, Davis and Januszkiewicz [DJ91, (5b.1) Theorem] constructed a topological locally CAT(0)-manifold, whose universal cover is not homeomorphic to $\mathbb{R}^{n}$. They also constructed a smooth locally CAT(0)-manifold whose universal cover is homeomorphic to $\mathbb{R}^{n}$, but the boundary at infinity is distinct from $S^{n-1}$ in (5c.1) Theorem.

More examples of those kind of exotic aspherical manifolds can be found in [ADG97] and [DJL12, Section 3].

Remark 2.8. Let $M^{n}(n \geq 5)$ be the locally CAT(0)-manifold with center-free fundamental group, whose universal cover is distinct from $\mathbb{R}^{n}$, as above-mentioned, and $N$ be an arbitrary closed locally CAT(0)-manifold with center-free fundamental group, then the product $M^{n} \times N$ is a locally CAT(0)-manifold, which does not support any non-positive curvature metrics. Otherwise, if $M^{n} \times N$ carries a non-positive curvature metric, then, by Lawson-Yau's splitting theorem [LY72], $M^{n} \times N$ is homeomorphic to $M_{1} \times N_{1}$ such that $M_{1}$ and $N_{1}$ are endowed with non-positive curvature metrics and $\pi_{1}\left(M_{1}\right)=\pi_{1}\left(M^{n}\right)$ and $\pi_{1}\left(N_{1}\right)=\pi_{1}(N)$. And then we use the proof of Borel conjecture for Riemannian manifold with non-positive sectional curvature by Farrell and Jones [FJ93] [FJ91] to show that $M_{1}$ is homeomorphic to $M^{n}$. Thus, $M_{1}$ also admits the locally CAT(0)-metric such that the 
universal cover is distinct from $\mathbb{R}^{n}$. That is a contradiction. More details of the proof can be found in [DJL12, Proposition 2].

Furthermore, using Davis' construction, Sapir [Sap14, Corollary 1.2] created the first closed aspherical topological $n$-manifolds $(n \geq 4)$ whose fundamental groups coarsely contain expanders and the aspherical $n$-manifold can be smooth if $n \geq 5$. Thus, Sapir's aspherical manifolds have infinite asymptotic dimension, are not coarsely embeddable into a Hilbert space, do not satisfy G. Yu's property A, do not satisfy the Baum-Connes conjecture with coefficients. Using Davis' construction and Sapir's techniques, Osajda constructed closed aspherical topological $n$-manifolds $(n \geq 4)$ whose fundamental groups contain coarsely embedded expanders in [Osa20, Corollary 3.5].

Our methods can be used to detected new manifolds (compared to Gromov-Lawson's method) which do not carry PSC-metrics. For example, the locally CAT(0)-metrics which were constructed by Davis-Januszkiewicz-Lafont above are strongly equivalent to a Riemannian metric on the exotic aspherical 4-manifolds. Therefore, the connected sum of arbitrary closed 4-manifold and a Davis-Januszkiewicz-Lafont exotic aspherical 4-manifold, is a new example detected by our methods, which carries no PSC-metrics.

Remark 2.9. A metric (piecewise flat) polyhedral complex which is both CAT(0) and a PL-manifold without boundary must in fact be PL-homeomorphic to the appropriate $\mathbb{R}^{n}$ was showed by Stone [Sto76, Theorem 1]. Thus, though the examples of Davis and Januskiewicz whose universal cover are not homeomorphic to $\mathbb{R}^{n}$ are piecewise Euclidean, their triangulation are not piecewise linear.

Remark 2.10. Siebenmann and Sullivan [SS79, Remark (1), p. 507]) showed that a locally finite simplicial complex with a polyhedral metric is a Lipschitz $n$-manifold if and only if the link of every vertex with the polyhedral metric is in turn a Lipschitz $(n-1)$-manifold and has the homotopy type of an $(n-1)$-sphere. The statement can also be found in [Lan19. Theorem 5.2]. Therefore, the locally CAT(0)-metric on a closed manifold, such that its 
universal cover is not homeomorphic to the appropriate $\mathbb{R}^{n}$, is not strongly equivalent to a Riemannian metric on the manifold.

It is not clear whether our methods can show that the connected sum of the locally CAT(0)-manifold, which is not strongly equivalent to a Riemannian manifold, and a closed manifold does not admit a PSC-metric.

\subsection{Enlargeable length-structures, PSC-metrics and lo- cally CAT(0)-metrics}

A metric space $(X, d)$ is a length metric space if the distance between each pair of points equals the infimum of the lengths of curves joining the points. A closed topological manifold admits length metrics [Bin53]. Metrics induced by smooth Riemannian metrics on a closed smooth manifold are length metrics and any two such length metrics are strongly equivalent, i.e. for any length metrics $d$ and $d^{\prime}$, there exists a $c, C>0$ such that $c d \leq d^{\prime} \leq C d$. Moreover, length metrics can be induced by continuous Riemannian metrics on a closed smooth manifold [Bur15].

However, unlike Riemannian metrics on compact smooth manifolds, different length metrics may not be strongly equivalent to each other. For instance, two length metrics, among which one is induced by a Riemannian metric and the other by a Finsher metric, are topologically equivalent, i.e. they induce the same topology, but there may not be strongly equivalent in general.

If $\pi: \hat{X} \rightarrow X$ is a covering map, then length metrics, being local, lift from $X$ to $\hat{X}$. Furthermore, every length metric $d$ on $X$ lifts to a unique length metric $\hat{d}$ for which the covering map is a local isometry.

We call that a topological manifold $X$ endowed with a complete length metric is $\varepsilon$ hyperspherical if it admits a continuous map $f$ to $S^{n}(n=\operatorname{dim}(X))$ which is constant at 
infinity, of non-zero degree and such that

$$
\operatorname{Lip}(f):=\sup _{\substack{a \neq b \\ a, b \in X}} \frac{d_{S^{n}}(f(a), f(b))}{d_{X}(a, b)}<\varepsilon .
$$

Here constant at infinity means that there is a compact subset such that $f$ maps the complement of the compact subset to a point in $S^{n}$ and $S^{n}$ is endowed with standard round metric. In this chapter, $S^{n}$ is also a length metric space induced by the standard round metric $d_{S^{n}}$.

Definition 2.11 (Enlargeable length-structures). A length metric $d$ on a closed orientable n-dimensional topological manifold $X^{n}$ is said to be enlargeable if for each $\varepsilon>0$ there is an oriented covering manifold $\widetilde{X^{n}}$ endowed with the induced metric $\widetilde{d}$ that is $\varepsilon$-hyperspherical.

An enlargeable length-structure on $X^{n}$ is a strongly equivalent class of an enlargeable metric.

An enlargeable Riemannian length-structure on a closed orientable smooth manifold is an enlargeable length-structure that contains a length metric induced by a Riemannian metric on the manifold.

The length metric induced by a Riemannian flat metric on $T^{n}$ is an enlargeable metric and $T^{n}$ endowed with this enlargeable length-structure is an important example of the manifolds with an enlargeable length-structure. Enlargeable length-structures can also be defined on a closed non-orientable manifold by lifting the metric onto the orientation cover.

Remark 2.12. The enlargeable length-structure may be used to deal with positive scalar curvature in the metric geometry setting. For instance, the definition of scalar curvature for length metrics was given in [Ver18].

Besides, the existence of a PSC-metric depends on the smooth structure. Trying to use length structure to study the PSC-metrics in Riemannian geometry, we define the enlargeable Riemannian length-structure. 
Remark 2.13. The following Lemma 2.20 shows that a locally CAT(0)-metric is enlargeable only on the assumption that it is strongly equivalent to a Riemannian metric. Especially, the example from the introduction of a non-smoothable manifold with a locally CAT(0)-metric is not known to be enlargeable.

Property 2.14 (Properties of the enlargeable metric).

(1) Let $\left(X, d_{X}\right)$ and $\left(Y, d_{Y}\right)$ be closed orientable manifolds with length metrics and suppose there exists a Lipschitz continuous map $F:\left(X, d_{X}\right) \rightarrow\left(Y, d_{Y}\right)$ of non-zero degree. If $d_{Y}$ is an enlargeable metric on $Y$, then so is $d_{X}$.

(2) The product of two enlargeable metrics is an enlargeable metric.

Proof. Suppose that $\widetilde{Y} \rightarrow Y$ is a covering space and $\widetilde{Y}$ is $\varepsilon$-hyperspherical, i.e. it admits a continuous map $f: \widetilde{Y} \rightarrow S^{n}(n=\operatorname{dim}(Y))$ that is constant at infinity, of non-zero degree and such that $\operatorname{Lip}(f)<\varepsilon$. Let $\widetilde{X} \rightarrow X$ be the covering corresponding to the subgroup $F_{*}^{-1}\left(\pi_{1}(\widetilde{Y})\right)$ and $\widetilde{X}$ be endowed with the induced metric $\widetilde{d_{X}}$, then $F$ can be lifted to a proper map $\widetilde{F}: \widetilde{X} \rightarrow \widetilde{Y}$, which is still a continuous map with $\operatorname{Lipschitz}$ constant $\operatorname{Lip}(F)$. It implies the map $f \circ \widetilde{F}$ is constant at infinity, $\operatorname{Lip}(f \circ \widetilde{F})<\operatorname{Lip}(F) \varepsilon$ and of non-zero degree. Thus $d_{X}$ is an enlargeable metric on $X$.

The fact that the composed map

$$
S^{n} \times S^{m} \rightarrow S^{n} \wedge S^{m} \rightarrow S^{n+m}
$$

is Lipschitz continuity and non-zero degree implies (2).

The following two corollaries are immediate consequences of Property 2.14 .

Corollary 2.15. If a closed manifold $X$ carries an enlargeable length-structure, then $X \times S^{1}$ still carries an enlargeable length-structure.

Corollary 2.16. Let $\left(X, d_{X}\right)$ be a closed smooth manifold, where $d_{X}$ is induced by a Riemannnian metric, and $\left(Y, d_{Y}\right)$ be another manifold, where $d_{Y}$ is in the enlargeable 
length-structure. Suppose there exists a Lipschitz continuous map $F:\left(X, d_{X}\right) \rightarrow\left(Y, d_{Y}\right)$ of non-zero degree, then $X$ carries an enlargeable Riemannian length-structure.

Theorem 2.17 (SLB Theroem [Gro18]). If the scalar curvature of a (possibly incomplete) Riemannian $n$-manifold $X^{n}(2 \leq n \leq 8)$ is bounded from below by $n(n-1)$, then for all continuous maps $f$ from $X^{n}$ to the sphere $S^{n}$ that are constant at infinity and of non-zero degree, they hold that $\operatorname{Lip}(f)>\frac{C}{\sqrt{n} \pi}$. Here $S^{n}$ is endowed with the standard round metric and $C>\frac{1}{3}$.

Question 2.18. Let $\left(N^{n}, g\right)$ be a complete Riemannian n-manifold (compact or noncompact) with scalar curvature bounded below by $n(n-1)$. Let $f$ be a continuous map from $N^{n}$ to the sphere $S^{n}$ with standard round metric of non-zero degree that is constant at infinity. Is $\operatorname{Lip}(f)$ bounded from blow by one?

Remark 2.19. A positive answer to Question 2.18 would allow us to also cover Llarull's rigidity theorems [Lla98, Theorem A and B], Lohkamp's results [Loh18], and their remarkable corollaries.

Gromov aimed to improve the lower bound of the Lipschitz constant in Gro18, Section 3] and discussed that the Lipschitz bound would be 1. Here, the existence of a uniformly positive lower bound of the Lipschitz constant is used as the main tool to detect the obstruction to the existence of PSC-metrics.

Proof of Theorem 2.1 Assume $X^{n}$ carries an enlargeable length-structure, there exists a length metric $d$ such that one orientable covering $\left(\widetilde{X^{n}}, \tilde{d}\right)$ is $\varepsilon$-hyperspherical $(\varepsilon$ small enough). If $X^{n}$ admits a PSC-metric $g$ in the enlargeable length-structure, then the Lipschitz constant of all maps (maps are constant at infinity and non-zero degree) from $\left(\widetilde{X^{n}}, \tilde{g}\right)$ to $S^{n}$ has a uniformly positive lower bound $C$ by the SLB Theorem. Besides, there are positive constants $\alpha_{1}$ and $\alpha_{2}$ such that $\alpha_{1} d \leq d_{g} \leq \alpha_{2} d$ by the definition of enlargeable length-structure, where $d_{g}$ is the induced metric by $g$ on $X^{n}$. Then the Lipschitz constant 
of the map from $\left(\widetilde{X^{n}}, \tilde{d}\right)$ to $S^{n}$ has the uniformly lower bound $\alpha_{1} C$, which contradicts the $\varepsilon$-hypersphericity.

Let $\left(X, d_{X}\right)$ be a length space. A geodesic triangle $\Delta$ in $X$ with geodesic segments as its sides is said to satisfy the CAT(0)-inequality if it is slimmer than the comparison triangle in the Euclidean plane, i.e. if there is a comparison triangle $\Delta^{\prime}$ in the Euclidean plane with sides of the same length as the sides of $\Delta$ such that the distance between points on $\Delta$ is less than or equal to the distance between corresponding points on $\triangle^{\prime}$. A length metric $d$ on $X$ is said to be a locally CAT(0)-metric if every point in $X$ has a geodesically convex neighborhood, in which every geodesic triangle satisfies the CAT(0)-inequality.

A locally CAT(0)-manifold is a topological manifold endowed with a locally CAT(0)metric. Gromov generalized the classic Hadamard-Cartan theorem to locally CAT(0)manifolds [BGS85]: the universal cover of a locally CAT(0)-manifold endowed with the induced metric is a globally CAT(0)-manifold, i.e. every two points can be connected by a unique geodesic line and every geodesic triangle on it satisfies the CAT(0)-inequality. Thus a locally $\mathrm{CAT}(0)$-manifold is aspherical, i.e. its universal cover is contractible.

Lemma 2.20. A locally $C A T(0)$-metric which is strongly equivalent to a Riemannian metric on a closed smooth manifold is an enlargeable metric.

Proof. Let $\left(M^{n}, d\right)$ be the closed $n$-dimensional smooth locally CAT(0)-manifold, then its universal cover $\left(\widetilde{M^{n}}, \tilde{d}\right)$ is a globally CAT(0)-manifold by Gromov's Theorem. Consider the map

$$
f_{t}: \widetilde{M^{n}} \rightarrow \widetilde{M^{n}} \quad x \rightarrow \gamma_{x}\left(t \tilde{d}\left(x, x_{0}\right)\right)
$$

where $x_{0}$ is a fixed point in $\widetilde{M^{n}}, t \in(0,1]$ and $\gamma_{x}$ is the unique geodesic segment from $x$ to $x_{0}$. It is well-defined by the property of globally CAT(0)-manifolds and is a proper map such that the degree of $f_{t}$ is non-zero. By the CAT(0)-inequality applied to the geodesic 
triangle with endpoints $x, y$ and $x_{0}$, one gets

$$
\tilde{d}\left(f_{t}(x), f_{t}(y)\right) \leq t d_{\mathbb{R}}^{2}(x, y)=t d(x, y)
$$

for $x, y \in \widetilde{M^{n}}$. Therefore, $\operatorname{Lip}\left(f_{t}\right) \leq t$.

Let $\pi:\left(\widetilde{M^{n}}, \tilde{d}\right) \rightarrow S^{n}$ be a collapse map around $x_{0}$. Then the degree of $\pi$ is 1 and $\operatorname{Lip}(\pi) \leq C$ by the smoothness of $\pi$, the fact that $d$ is strongly equivalent to a Riemannian metric and the compactness of $M^{n}$. Thus, $\pi \circ f_{t}: \widetilde{M^{n}} \rightarrow S^{n}$ has non-zero degree and $\operatorname{Lip}\left(\pi \circ f_{t}\right) \leq t C$. For any small $\varepsilon>0$, we can choose $t$ such that $\left(\widetilde{M^{n}}, \tilde{d}\right)$ is $\varepsilon$-hyperspherical. That means that a manifold endowed with a locally CAT(0)-metric is enlargeable.

Remark 2.21. The assumption that the locally CAT(0)-metric is strongly equivalent to a Riemannian metric is used in the argument of $\operatorname{Lip}(\pi) \leq C$. It is not clear if the condition can be dropped for Lemma 2.20 .

Proof of Theorem 2.5. Combining Lemma 2.20, Corollary 2.16, and the fact of the strong equivalence of all Riemannian metrics on a closed smooth manifold, we conclude Theorem 2.5 .

\section{3 $S c^{M V} \geq \kappa$}

To generalize the notion of PSC-metrics to non-Riemannian metric space, for example to piecewise smooth polyhedral spaces, Gromov [Gro19, Section 5.3.1] defined the maxscalar curvature $S c^{\max }$ as follows:

Definition 2.22 (Gromov). Given a metric space $P$ which is locally compact and locally contractible, and a homology class $h \in H_{n}(P)$ defines $S c^{\max }(h)$ as the supremum of the numbers $\sigma \geq 0$, such that there exists a closed orientable Riemannian $n$-manifold $X$ with $S c(X) \geq \sigma$ and a 1-Lipschitz map $f: X \rightarrow P$, such that the fundamental homology class 
$[X]$ goes to $h, f_{*}[X]=h$. Here $S c(X)$ is the scalar curvature of $X$ and 1-Lipschitz map $f$ means $\operatorname{Lip}(f) \leq 1$.

Remark 2.23. The definition makes sense without assuming $\sigma \geq 0$. But if an $h \in H_{n}(P)$ $(n \geq 3)$ can be represented by the fundamental homology class $[X]$, then we always have $S c^{\max }(h) \geq 0$. Since a closed orientable smooth $n$-manifold $(n \geq 3)$ admits Riemnanian metrics with constant negative scalar curvature by Kazdan-Warner theorem [KW75], then one can scale the metric such that the Lipschitz constant is small and the scalar curvature is closed to 0 .

Though $S c^{\max }([X]) \geq \inf _{x} S c(X, x)$ for all closed Riemannian manifold $X$ as observed by Gromov, the positivity of the max-scalar curvature cannot imply that it carries a PSC-metric in general. For instance, let $\Sigma$ be the exotic sphere which does not admit PSC-metrics, whose existence was showed by Hitchin in [Hit74], and $g_{\Sigma}$ be a Riemanniam metric on it. Then one can scale the round metric on the standard sphere such that the Lipschitz constant of the identity map is smaller than 1 . Thus one has $S c^{\max }([\Sigma])>0$, but $\Sigma$ does not admit PSC-metrics.

Furthermore, let $\left(N, g_{N}\right)$ be a closed oriented Riemannian manifold with a PSC-metric $g_{N}$ and $M$ be a closed oriented smooth manifold. Assume that there exists a degree one smooth map $f: N \rightarrow M$, then one gets $S c^{\max }([M]) \geq 0$ with an arbitrary smooth Riemannian metric $g$ on $M$. That means $S c^{\max }([M]) \geq 0$, even when the scalar curvature of $(M, g)$ is -1 .

Therefore, the definition of the max-scalar curvature on a metric space needs to be improved. Gromov also proposed the $n$-volumic scalar curvature on metric measure spaces in [Gro17, Section 26]. The $n$-volumic scalar curvature may be too general to be useful and we will discuss it in the next chapter. The following definition is trying to mix the max-scalar curvature and $n$-volumic scalar curvature on metric measure spaces.

Let $\left(S^{2}(R), d_{S}, \operatorname{vol}_{S}\right)$ be a Riemannian 2-sphere endowed with the round metric such 
that the scalar curvature equals to $2 R^{-2}$ and that $\left(\mathbb{R}^{n-2}, d_{E}, \operatorname{vol}_{E}\right)$ is endowed with Euclidean metric, then the product manifold $S^{2}(R) \times \mathbb{R}^{n-2}$ can be endowed with the Pythagorean product metrics $d_{S \times E}:=\sqrt{d_{S}^{2}+d_{E}^{2}}$ and the volume $\operatorname{vol}_{S \times E}:=\operatorname{vol}_{S} \otimes \operatorname{vol}_{E}$.

In this chapter, let $X^{n}$ be a closed topological $n$-manifold and $d$ be the length metric such that the Hausdorff dimension of $\left(X^{n}, d\right)$ is $n$, i.e $\operatorname{dim}_{H}\left(X^{n}\right)=n$. Therefore, there exists the $n$-dimension Hausdorff measure $\mu_{n}$ on it and then we normalize it by $\mathcal{H}_{d}^{n}=\mathcal{W}_{n} \mu_{n}$, where $\mathcal{W}_{n}$ is the $n$-dimensional volume (the Lebesgue measure) of a Euclidean ball of radius 1 in the $n$-dimensional Euclidean space, so that $\mathcal{H}_{d_{E}}^{n}=\operatorname{vol}_{E}$ for $\mathbb{R}^{n}$ with the Euclidean metric $d_{E}$. Therefore, we have the metric measure space $\left(X^{n}, d, \mathcal{H}_{d}^{n}\right)$ and will focus on this kind of metric measure space $\left(X^{n}, d, \mathcal{H}_{d}^{n}\right)$ in this paper.

Example 2.24 (Examples of metric measure spaces $\left.\left(X^{n}, d, \mathcal{H}_{d}^{n}\right)\right)$.

1. Smooth oriented Riemannian manifolds $\left(M^{n}, g\right)$ with the induced volume forms $\left(M^{n}, d_{g}, \operatorname{vol}_{g}\right)$ are the fundamental examples.

2. A length metric that is strongly equivalent to a Riemannian metric satisfies the requirement, since the Hausdorff dimension is bi-Lipschitz invariant.

3. Locally CAT(0)-manifolds with induced Hausdorff measure are examples, as the Hausdorff dimension of a closed topological $n$-manifold with a locally CAT( 0$)$-metric is $n$.

4. If $X^{n}$ admits an length metric $d^{\prime}$ such that $\left(X^{n}, d^{\prime}\right)$ is a Alexandrov space with curvature bounded from below, then $\operatorname{dim}_{H}\left(X^{n}\right)=n$. Then the Alexandrov space is also an example.

Note that there is only one reasonable notion of volume for Riemannian manifolds. But one can define Finslerian volumes for Finsler metrics in different ways and obtain essentially different results [BBI01, Proposition 5.5.12]. 
We will define the positive of $M V$-scalar curvature $S c^{M V}$ on the metric measure space $\left(X^{n}, d, \mathcal{H}_{d}^{n}\right)$.

Definition 2.25 $\left(S c^{M V} \geq \kappa\right)$. The $M V$-scalar curvature of $X^{n}$ is bounded from below by $\kappa>0$ for $X^{n}=\left(X^{n}, d, \mathcal{H}_{d}^{n}\right)$, i.e. $S c^{M V}\left(X^{n}\right) \geq \kappa>0$, if the closed oriented topological $n$-dimensional $X^{n}$ satisfies the following two conditions:

(1). The metric space $\left(X^{n}, d\right)$ satisfies $S c^{\max }\left(\left[X^{n}\right]\right) \geq \kappa$ for the fundamental class $\left[X^{n}\right] \in$ $H_{n}\left(X^{n} ; \mathbb{Z}\right)$.

(2). The metric measure space $X^{n}$ is locally volume-wise smaller than $S^{2}(R) \times \mathbb{R}^{n-2}=$ $\left(S^{2}(R) \times \mathbb{R}^{n-2}, d_{S \times E}, \operatorname{vol}_{S \times E}\right)$ for all $R>\frac{\sqrt{2}}{\sqrt{\kappa}}$, i.e. for $R>\frac{\sqrt{2}}{\sqrt{\kappa}}$, there is an $\epsilon_{R}$, which depends on $R$, such that all $\epsilon_{R}$-balls in $X$ are smaller than the $\epsilon_{R}$-balls in $S^{2}(R) \times \mathbb{R}^{n-2}$, $\mathcal{H}_{d}^{n}\left(B_{x}\left(\epsilon_{R}\right)\right)<\operatorname{vol}_{S \times E}\left(B_{x^{\prime}}\left(\epsilon_{R}\right)\right)$, for all $x \in X$ and $x^{\prime} \in S^{2}(R) \times \mathbb{R}^{n-2}$.

Since $S c^{\max }\left(\left[X^{n}\right]\right)=S c^{\max }\left(-\left[X^{n}\right]\right)$, the definition is independent from the chosen of the orientation. And $S c^{M V}\left(X^{n}\right) \geq \kappa$ is invariant under the isomorphic transformation. Here an isomorphic transformation means there is $f:(X, d, \mu) \rightarrow\left(X^{\prime}, d^{\prime}, \mu^{\prime}\right)$ such that $f_{*} \mu^{\prime}=\mu$ and $f$ is isometric between $d$ and $d^{\prime}$. Thus, the definition is well-defined.

Proposition 2.26. Let $g$ be a $C^{2}$-smooth Riemannian metric on a closed oriented $n$ manifold $M^{n}$ with induced metric measure space $\left(M^{n}, d_{g}, \operatorname{vol}_{g}\right)$, then the scalar curvature of $g$ is bounded from below by $\kappa>0$ if and only if $S c^{M V}\left(M^{n}\right) \geq \kappa$.

Proof. Assume the scalar curvature of $g$ is bounded from below by $\kappa>0$, then the volume formula of a small ball,

$$
\operatorname{vol}_{g}\left(B_{x}(r)\right)=\operatorname{vol}_{E}\left(B_{r}\right)\left[1-\frac{S c_{g}(x)}{6(n+2)} r^{2}+O\left(r^{4}\right)\right]
$$

as $r \rightarrow 0$, where $B_{x}(r)$ is an $r$-ball in $M^{n}$ and $B_{r}$ is an $r$-ball in $\mathbb{R}^{n}$, implying condition (2) in the definition of $S c^{M V}\left(M^{n}\right) \geq \kappa$. And $S c^{\max }\left(\left[M^{n}\right]\right) \geq \inf _{x} S c\left(M^{n}, x\right) \geq \kappa$ implies condition (1). 
On the other hand, if $S c^{M V}\left(M^{n}\right) \geq \kappa$, then $S c_{g} \geq \kappa>0$. Otherwise, assume there exist small $\epsilon>0$ such that $S c_{g} \geq \kappa-\epsilon>0$. That means there exists a point $x_{0}$ in $M^{n}$ such that $S c_{g}\left(x_{0}\right)=\kappa-\epsilon$, as $M^{n}$ is compact and the scalar curvature is a continuous function on $M^{n}$. Thus, we can find a small $r$-ball $B_{r}\left(x_{0}\right)$ such that the volume of $B_{r}\left(x_{0}\right)$ is greater than the volume of the $r$-ball in the $S^{2}(\gamma) \times \mathbb{R}^{n-2}$ for $\gamma=\sqrt{\frac{2}{\kappa-\frac{\epsilon}{2}}}$, which is a contradiction.

Remark 2.27. The existence of length metrics on $X^{n}$ with $S c^{M V}\left(X^{n}\right) \geq \kappa$ is the invariant under homeomorphisms. However, the positivity of the $S c^{M V}$ cannot imply that it carries a PSC-metric in general.

For instance, one can use the identity map between the exotic sphere $\Sigma^{n}$ above and the standard sphere to pull back the length metric which is induced by the standard round metric to $\Sigma^{n}$, i.e. giving $i d: \Sigma^{n} \rightarrow\left(S^{n}, d_{S^{n}}\right)$ gets $\left(\Sigma^{n}, i d^{*} d_{S^{n}}\right)$. Then one has $S c^{M V}\left(\Sigma^{n}\right) \geq n(n-1)$ for the metric measure space $\left(\Sigma^{n}, i d^{*} d_{S^{n}}, \mathcal{H}_{i d^{*} d_{S^{n}}}^{n}\right)$. But $i d^{*} d_{S^{n}}$ is not induced by any $C^{2}$-smooth Riemannian metric on $\Sigma^{n}$.

Remark 2.28. The example of the exotic sphere above shows that the condition (1) cannot imply the condition (2) in the definition of $S c^{M V} \geq \kappa$ in general. The condition (2) also cannot imply the condition (1) in general. Since one can choose the length metric induced by a Finsler metric such that the induced Hausdorff measure is locally volume-wise smaller than $S^{2}(R) \times \mathbb{R}^{n-2}=\left(S^{2}(R) \times \mathbb{R}^{n-2}, d_{S \times E}, \operatorname{vol}_{S \times E}\right)$ for all $R>\frac{\sqrt{2}}{\sqrt{\kappa}}$, and the Finsler metric is not bi-Lipshchitz equivalent to a Riemannian metric in general.

Question 2.29. Let $N^{n}$ be a closed orientable aspherical n-manifold. Does there exist an orientable closed Riemannian n-manifold $M^{n}$ such that there exists a non-zero degree map from $M^{n}$ to $N^{n}$ ?

Remark 2.30. Question 2.29 is inspired by the conjecture that a closed aspherical manifold does not carry PSC-metrics. It is natural to ask that whether a closed aspherical manifold admits a length metric with positive max-scalar curvature or positive of $S c^{M V}$. 
Proposition 2.31 (Quadratic Scaling). Assume that $\left(X^{n}, d, \mathcal{H}_{d}^{n}\right)$ satisfies $S c^{M V}\left(X^{n}\right) \geq \kappa>0$, then $S c^{M V}\left(\lambda X^{n}\right) \geq \lambda^{-2} \kappa$ for all $\lambda>0$, where $\lambda X^{n}:=\left(X^{n}, \lambda \cdot d, \lambda^{n} \cdot \mathcal{H}_{d}^{n}\right)$.

Proof. If we scale $d$ by $\lambda \neq 0$, then $\mathcal{H}_{\lambda d}^{n}=\lambda^{n} \mathcal{H}_{d}^{n}$. Combining it with the fact that the $S c\left(\lambda^{2} g\right)=\lambda^{-2} S c(g)$ for a smooth Riemannian metric will complete the proof.

Let $\pi: \hat{X}^{n} \rightarrow X^{n}$ be a covering map, then the length metric $d$ on $X^{n}$ is lifted to a unique length metric $\hat{d}$ such that the covering map is a local isometry. Hence $\operatorname{dim}_{H}(X, d)=$ $\operatorname{dim}_{H}(\hat{X}, \hat{d})$ for a finite connected cover of $X$. Then we endow the lifting length metric on a finite cover of $\left(X^{n}, d, \mathcal{H}_{d}^{n}\right)$ such that $\left(\hat{X}^{n}, \hat{d}, \mathcal{H}_{\hat{d}}^{n}\right)$ is a metric measure space.

Proposition 2.32. Assume $\left(X^{n}, d, \mathcal{H}_{d}^{n}\right)$ satisfies $S c^{M V}\left(X^{n}\right) \geq \kappa>0$ and $\hat{X}^{n}$ is a finite connected cover of $X^{n}$, then $S c^{M V}\left(\hat{X}^{n}\right) \geq \kappa$ for $\left(\hat{X}^{n}, \hat{d}, \mathcal{H}_{\hat{d}}^{n}\right)$.

Proof. As $\left(\hat{X}^{n}, \hat{d}, \mathcal{H}_{\hat{d}}^{n}\right)$ is locally isometric to $\left(X^{n}, d, \mathcal{H}_{d}^{n}\right)$ and $S c^{M V}\left(X^{n}\right) \geq \kappa>0,\left(\hat{X}^{n}, \hat{d}, \mathcal{H}_{\hat{d}}^{n}\right)$ is also locally volume-wise smaller than $S^{2}(R) \times \mathbb{R}^{n-2}$.

Let $\left(M^{n}, g\right)$ be the closed orientable Riemannian manifold with $S c(g) \geq \kappa$ such that $f: M^{n} \rightarrow X^{n}$ is 1-Lipschitz map and $f_{*}\left(\left[M^{n}\right]\right)=\left[X^{n}\right]$. Then $f^{*} \hat{X}^{n}$ is a finite cover of $M^{n}$ and we denote it by $\hat{M}^{n}$, i.e. $\hat{M}^{n}:=f^{*} \hat{X}^{n}$. Then the Lipshicht constant of $\hat{f}:\left(\hat{M}^{n}, \hat{g}\right) \rightarrow\left(\hat{X}^{n}, \hat{d}\right)$ is 1 , where $\hat{g}$ is the lifting metric of $g$. Then we have the following two commutative diagrams.
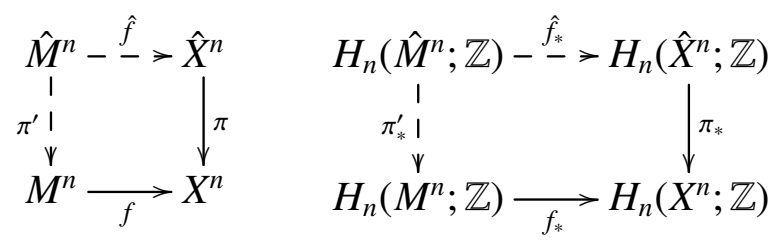

Using the wrong-way map, we can map $\left[X^{n}\right]$ to $H_{n}\left(\hat{X}^{n} ; \mathbb{Z}\right)$, denoting it by $\left[\hat{X}^{n}\right]$ and then we choose $\left[\hat{X}^{n}\right]$ as the fundamental class of $\hat{X}^{n}$. Again, we map $\left[\hat{X}^{n}\right]$ to $H_{n}\left(\hat{M}^{n} ; \mathbb{Z}\right)$, denoting it by $\left[\hat{M}^{n}\right]$ and choose $\left[\hat{M}^{n}\right]$ as the fundamental class of $\hat{M}^{n}$, i.e. $\hat{f}_{*}\left(\left[\hat{M}^{n}\right]\right)=\left[\hat{X}^{n}\right]$.

Thus, $S c^{M V}\left(\hat{X}^{n}\right) \geq \kappa$. 
Remark 2.33. One can also define $S c^{M V} \geq \kappa>0$ for closed non-orientable topological manifolds by requiring that the double cover with the induced metric satisfies $S c^{M V} \geq \kappa>0$, since the Hausdorff dimensions are equal to each other in this case.

Proposition 2.34 ( Weak SLB Theorem). Assume $\left(X^{n}, d, \mathcal{H}_{d}^{n}\right)(n \leq 8)$ satisfies $S c^{M V}\left(X^{n}\right) \geq$ $\kappa>0$, then for all continuous non-zero degree maps $h$ from $X^{n}$ to the sphere $S^{n}$, it holds that $\operatorname{Lip}(h)>\frac{C \sqrt{n-1}}{\sqrt{\kappa} \pi}$. Here $S^{n}$ is endowed with the standard round metric $d_{S^{n}}$ and $C>\frac{1}{3}$.

Proof. Given a small $\epsilon>0$, there exists a closed orientable Riemannian $n$-manifold $\left(M^{n}, g\right)$ with $S c(g) \geq \kappa-\epsilon>0$ and a 1-Lipschitz and degree one map $f:\left(M^{n}, d_{g}\right) \rightarrow\left(X^{n}, d\right)$ by the definition of max-scalar curvature. Let $h$ be a continuous non-zero degree map $h:\left(X^{n}, d\right) \rightarrow\left(S^{n}, d_{S^{n}}\right)$, then

$$
\operatorname{Lip}(h \circ f) \leq \operatorname{Lip}(h) \times \operatorname{Lip}(f)
$$

induces $\operatorname{Lip}(h) \geq \operatorname{Lip}(h \circ f)$.

If one scalars the metrics $d_{g}$ and $d$ by a constant $\lambda \neq 0$, then one has $S c\left(\lambda^{2} g\right)=$ $\lambda^{-2} S c(g), d_{\lambda^{2} g}=\lambda d_{g}$. The new maps are denoted by $\tilde{h}:\left(X^{n}, \lambda d\right) \rightarrow\left(S^{n}, d_{S^{n}}\right)$ and $\tilde{f}:\left(M^{n}, \lambda d_{g}\right) \rightarrow\left(X^{n}, d\right)$, then one has $\operatorname{Lip}(\tilde{f})=\operatorname{Lip}(f), \operatorname{Lip}(\tilde{h} \circ \tilde{f})=\lambda^{-1} \operatorname{Lip}(h \circ f)$ and $\operatorname{Lip}(\tilde{h})=\lambda^{-1} \operatorname{Lip}(h)$.

Choose $\lambda=\sqrt{\frac{n(n-1)}{\kappa-\epsilon}}$ such that $S c\left(\lambda^{2} g\right) \geq n(n-1)$, then SLB Theorem implies that $\operatorname{Lip}(\tilde{h} \circ \tilde{f})>\frac{C}{\sqrt{n} \pi}$. That means

$$
\lambda^{-1} \operatorname{Lip}(h)=\operatorname{Lip}(\tilde{h}) \geq \operatorname{Lip}(\tilde{h} \circ \tilde{f})>\frac{C}{\sqrt{n} \pi} .
$$

Thus, we have $\operatorname{Lip}(h)>\frac{C \sqrt{n-1}}{\sqrt{\kappa-\epsilon \pi}}$. Let $\epsilon$ go to 0 , then one has $\operatorname{Lip}(h)>\frac{C \sqrt{n-1}}{\sqrt{\kappa} \pi}$.

Gromov and Lawson define the enlargeability by allowing only finite coverings in [GL80]. Hanke and Schick [HS06] showed that the Rosenberg index of this kind of enlargeable spin manifold does not vanish, which also implies that the manifold carries no PSC-metrics. 
Definition 2.35 (Compactly enlargeable length-structures). A length metric $d$ on a closed orientable n-dimensional topological manifold $X^{n}$ is said to be compactly enlargeable if for each $\varepsilon>0$ there is a finite connected covering manifold $\hat{X}^{n}$ endowed with the induced metric $\hat{d}$ which is $\varepsilon$-hyperspherical. (This notion is defined at the Definition 2.11).

A compactly enlargeable length-structure on $X^{n}$ is the strongly equivalent class of an enlargeable metric.

A compactly enlargeable Riemannian length-structure on a closed orientable smooth manifold is an enlargeable length-structure, which contains a length metric induced by a Riemannian metric on the manifold.

Let us recall Theorem 2.4 and give a proof of it.

Theorem 2.36. If $\left(X^{n}, d, \mathcal{H}_{d}^{n}\right)(n \leq 8)$ satisfies $S c^{M V}\left(X^{n}\right) \geq \kappa>0$, then $d$ is not in any compactly enlargeable length-structures on the closed oriented topological manifold X.

Proof. The argument is the same in the proof of Theorem 2.1. Assume $d$ is in a compactly enlargeable length-structure, then there exists a compactly enlargeable metric $d^{\prime}$ on $X^{n}$ such that $\alpha_{1} d \leq d^{\prime} \leq \alpha_{2} d$ for some $0<\alpha_{1} \leq \alpha_{2}$. There exists a finite connected covering manifold $\hat{X}^{n}$ such that the induced metric $\hat{d}^{\prime}$ ) is $\varepsilon$-hyperspherical for $\varepsilon<\frac{C \sqrt{n-1}}{\sqrt{\kappa} \alpha_{2} \pi}$ by the definition of compactly enlargeable metric. That means it exists the non-zero degree map $h:\left(\hat{X}, \hat{d}^{\prime}\right) \rightarrow S^{n}$ such that $\operatorname{Lip}(h)_{\hat{d}^{\prime}}<\frac{c \sqrt{n-1}}{\sqrt{\kappa} \alpha_{2} \pi}$, where $\operatorname{Lip}(h)_{\hat{d}^{\prime}}$ is the Lipschitz constant with respect the metric $\hat{d}^{\prime}$.

On the other hand, we have

$$
\alpha_{2}^{-1} \operatorname{Lip}(h)_{\hat{d}} \leq \operatorname{Lip}(h)_{\hat{d}^{\prime}} \leq \alpha_{1}^{-1} \operatorname{Lip}(h)_{\hat{d}}
$$

But Proposition 2.32 shows that $S c^{M V}\left(\hat{X}^{n}, \hat{d}\right) \geq \kappa$ and then Weak SLB Theorem 2.34 shows that $\operatorname{Lip}(h)_{\hat{d}}>\frac{C \sqrt{n-1}}{\sqrt{\kappa} \pi}$, which is a contradiction.

One can also prove the proposition by Corollary 2.16 and the definition of max-scalar curvature. If $d$ is in a compactly enlargeable length-structure and $f:\left(M^{n}, g\right) \rightarrow\left(X^{n}, d\right)$ 
is a degree 1 map and $\operatorname{Lip}(f) \leq 1$, then $g$ is in an enlargeable Riemannian length-metric structure by Corollary 2.16. That means $M^{n}$ carries no PSC-metrics. Thus, $S c^{\max }\left(\left[X^{n}\right]\right)=0$, which is a contradiction.

The scalar curvature of Riemannian metrics are additivity under Pythagorean-Riemannian products, however, $S c^{M V}$ may not be additivity under Pythagorean products in general. Note that for the Pythagorean product of two metric spaces $(X, d)$ and $\left(Y, d_{1}\right),\left(X \times Y, d_{2}\right)$ with $d_{2}:=\sqrt{d^{2}+d_{1}^{2}}$, we have

$$
\operatorname{dim}_{H}(X)+\operatorname{dim}_{H}(Y) \leq \operatorname{dim}_{H}(X \times Y) \leq \operatorname{dim}_{H}(X)+\operatorname{dim}_{B}(Y),
$$

where $\operatorname{dim}_{B}(Y)$ is the upper box counting dimension of $Y$, and the inequality may be strict. If $Y$ is a smooth Riemannian manifold, then $\operatorname{dim}_{H}(Y)=\operatorname{dim}_{B}(Y)$. Furthermore, we have

$$
\mathcal{H}_{d_{2}}^{n+m} \geq C(n, m) \mathcal{H}_{d}^{n} \times \mathcal{H}_{d_{1}}^{m}
$$

where $C(n, m)$ is a constant dependent only on $n$ and $m$ and $C(n, m) \geq 1$. $C(n, m)$ may be greater than 1, but if $X$ and $Y$ are rectifiable Borel subsets of Euclidean space, then $C(n, m)=1$ was showed by Federer in [Fed69, 3.2.23 Theorem].

Proposition 2.37. Assume $\left(X^{n} \times Y^{m}, d_{2}\right)$ is the Pythagorean product of $\left(X^{n}, d, \mathcal{H}_{d}^{n}\right)$ and $\left(Y^{m}, d_{1}, \mathcal{H}_{d_{1}}^{n}\right)$, where $d_{2}:=\sqrt{d^{2}+d_{1}^{2}}$, satisfies that $\operatorname{dim}_{H}\left(X^{n} \times Y^{m}\right)=n+m$ and the measure $\mathcal{H}_{d_{2}}^{n+m}:=\mathcal{H}_{d}^{n} \otimes \mathcal{H}_{d_{1}}^{m}$. Then if $S c^{M V}\left(X^{n}\right) \geq \kappa_{1}>0$ and $S c^{M V}\left(Y^{m}\right) \geq \kappa_{2}>0$, then $S c^{M V}\left(X^{n} \times Y^{m}\right) \geq \kappa_{1}+\kappa_{2}$.

Proof. Since $\left(X^{n} \times Y^{m}, d_{2}\right)$ is locally volume-wise smaller than $\left(S^{2}\left(R_{1}\right) \times \mathbb{R}^{n-2}\right) \times\left(S^{2}\left(R_{2}\right) \times\right.$ $\mathbb{R}^{m-2}$ ) for $R_{1}>\frac{\sqrt{2}}{\sqrt{\kappa_{1}}}$ and $R_{2}>\frac{\sqrt{2}}{\sqrt{\kappa_{2}}}$, since $\mathcal{H}_{d_{2}}^{n+m}:=\mathcal{H}_{d}^{n} \otimes \mathcal{H}_{d_{1}}^{m}$. And $\left(S^{2}\left(R_{1}\right) \times \mathbb{R}^{n-2}\right) \times\left(S^{2}\left(R_{2}\right) \times\right.$ $\left.\mathbb{R}^{m-2}\right)$ is locally volume-wise smaller than $S^{2}\left(R_{1}+R_{2}\right) \times \mathbb{R}^{n+m-2}$ for $R_{1}+R_{2}>\frac{\sqrt{2}}{\sqrt{\kappa_{1}+\kappa_{2}}}$. Thus, we have $\left(X^{n} \times Y^{m}, d_{2}\right)$ is locally volume-wise smaller than $S^{2}\left(R_{1}+R_{2}\right) \times \mathbb{R}^{n+m-2}$ for $R_{1}+R_{2}>\frac{\sqrt{2}}{\sqrt{\kappa_{1}+\kappa_{2}}}$. And one has

$$
S c^{\max }\left(\left[X^{n}\right] \otimes\left[Y^{m}\right]\right) \geq S c^{\max }\left(\left[X^{n}\right]\right)+S c^{\max }\left(\left[Y^{m}\right]\right)
$$


Hence $S c^{\max }\left(\left[X^{n}\right] \otimes\left[M^{m}\right]\right) \geq \kappa_{1}+\kappa_{2}$.

Question 2.38. Assume that $d$ is a length metric on the closed topological n-manifold $X^{n}$ such that $\left(X^{n}, d\right)$ is an Alexandrov space with curvature $\geq \kappa>0$. Do we have $S c^{M V}\left(\left[X^{n}\right]\right) \geq$ $n(n-1) \kappa ?$

Note that an Alexandrov space with curvature $\geq \kappa>0$ satisfies the Bishop-inequality [BBI01, Theorem 10.6.8] and then it implies the condition (2) of the definition of $S c^{M V} \geq \kappa$. 


\section{Curvature-Dimension Condition}

\section{Meets Gromov's $n$-Volumic Scalar}

\section{Curvature}

We study the properties of the $n$-volumic scalar curvature in this chapter. Lott-SturmVillani's curvature-dimension condition $C D(\kappa, n)$ [LV09], [Stu06a], [Vil09] was showed to imply the $n$-volumic scalar curvature $\geq n \kappa$ under an additional $n$-dimensional condition and we show the stability of $n$-volumic scalar curvature $\geq \kappa$ with respect to smGH-convergence.

The concept of lower bounded curvature on the metric space or the metric measure space has evolved to a rich theory due to Alexandrov's insight. The stability of Riemannian manifolds with curvature bounded below is another deriving force to extend the definition of the curvature bounded below to a broader space.

However, the scalar curvature (of Riemannian metric) bounded below was yet absent from this picture. Gromov proposed a synthetic treatment of scalar curvature bounded below, which was called the $n$-volumic scalar curvature bounded below, and offered some pertinent conjectures in [Gro17, Section 26]. Motivated by the $C D(\kappa, n)$ condition, we add an $n$-dimension condition to the Gromov's definition and introduce the definition of $S c_{\alpha, \beta}$ on the smooth metric measure space.

This chapter comes from my published paper [Den21a]. 


\subsection{CD meets $n$-volumic scalar curvature}

The $n$-dimensional Alexandrov space with curvature $\geq \kappa$ equipped with the volumemeasure satisfies Lott-Villani-Sturm's weak curvature-dimension condition for dimension $n$ and curvature $(n-1) \kappa$, i.e. $C D((n-1) \kappa, n)$, was shown by Petrunin for $\kappa=0$ (and said that for general curvature $\geq \kappa$ the result followed in a similar way) [Pet11] and then Zhang-Zhu investigated the general case [ZZ10]. We will modify Gromov's definition of $n$-volumic scalar curvature bounded below in [Gro17, Section 26] to fill the picture, which means Lott-Sturm-Villani's Ricci curvature $\geq 0$ implies Gromov's scalar curvature $\geq 0$.

The metric measure space (mm-space) $X=(X, d, \mu)$ means that $d$ is the complete separable length metric on $X$ and $\mu$ is the locally finite full support Borel measure on $X$ equipped with its Borel $\sigma$-algebra. Say that an mm-space $X=(X, d, \mu)$ is locally volume-wise smaller (or not greater) than another such space $X^{\prime}=\left(X^{\prime}, d^{\prime}, \mu^{\prime}\right)$ and write $X<_{\text {vol }} X^{\prime}\left(X \leq_{\text {vol }} X^{\prime}\right)$, if all $\epsilon$-balls in $X$ are smaller (or not greater) than the $\epsilon$-balls in $X^{\prime}$, $\mu\left(B_{\epsilon}(x)\right)<\mu^{\prime}\left(B_{\epsilon}\left(x^{\prime}\right)\right)\left(\mu\left(B_{\epsilon}(x)\right) \leq \mu^{\prime}\left(B_{\epsilon}\left(x^{\prime}\right)\right)\right.$, for all $x \in X, x^{\prime} \in X^{\prime}$ and the uniformly small $\epsilon$ which depends on $X$ and $X^{\prime}$.

In this chapter, the Riemannian 2-sphere $\left(S^{2}(\gamma), d_{S}, \operatorname{vol}_{S}\right)$ is endowed with round metric such that the scalar curvature equal to $2 \gamma^{-2},\left(\mathbb{R}^{n-2}, d_{E}, \operatorname{vol}_{E}\right)$ is endowed with Euclidean metric with flat scalar curvature and the product manifold $S^{2}(\gamma) \times \mathbb{R}^{n-2}$ is endowed with the Pythagorean product metrics $d_{S \times E}:=\sqrt{d_{S}^{2}+d_{E}^{2}}$ and the volume $\operatorname{vol}_{S \times E}:=\operatorname{vol}_{S} \otimes \operatorname{vol}_{E}$.

Thus, we have $S^{2}(\gamma)<_{v o l} \mathbb{R}^{2}$ for all $\epsilon$-balls and $\epsilon \leq \gamma \pi$. If $0<\gamma_{1}<\gamma_{2}$, then $S^{2}\left(\gamma_{1}\right)<_{\text {vol }} S^{2}\left(\gamma_{2}\right)$. Furthermore, $S^{2}(\gamma) \times \mathbb{R}^{n-2}<_{\text {vol }} \mathbb{R}^{n}$. If $0<\gamma_{1}<\gamma_{2}$, then $S^{2}\left(\gamma_{1}\right) \times$ $\mathbb{R}^{n-2}<_{\text {vol }} S^{2}\left(\gamma_{2}\right) \times \mathbb{R}^{n-2}$.

Definition 3.1 (Gromov's $n$-volumic scalar curvature). Gromov's $n$-volumic scalar curvature of $X$ is bounded below by 0 for $X=(X, d, \mu)$ if $X$ is locally volume-wise not greater than $\mathbb{R}^{n}$.

Gromov's $n$-volumic scalar curvature of $X$ bounds from below by $\kappa>0$ for $X=(X, d, \mu)$ 
if $X$ is locally volume-wise smaller than $S^{2}(\gamma) \times \mathbb{R}^{n-2}$ for all $\gamma>\sqrt{\frac{2}{\kappa}}$, i.e. $X<_{\text {vol }} S^{2}(\gamma) \times \mathbb{R}^{n-2}$ and $\gamma>\sqrt{\frac{2}{\kappa}}$, where $S^{2}(\gamma) \times \mathbb{R}^{n-2}=\left(S^{2}(\gamma) \times \mathbb{R}^{n-2}, d_{S \times E}, \operatorname{vol}_{S \times E}\right)$.

The $n$-volumic scalar curvature is sensitive to the scaling of the measure, but, the curvature condition $C D(\kappa, n)$ of Lott-Villani-Sturm [Stu06b, Definition 1.3] is invariant up to scalars of the measure only [Stu06b, Proposition 1.4 (ii)]. Therefore, the $n$-dimensional condition needs to be put into the definition of Gromov's $n$-volumic scalar curvature. In fact, the $n$-dimensional condition is the special case of Young's point-wise dimension in dynamical systems [YYou82, Theorem 4.4].

Definition 3.2 ( $n$-dimensional condition). For given positive natural number $n$, the mmspace $X=(X, d, \mu)$ satisfies the $n$-dimensional condition if

$$
\lim _{r \rightarrow 0} \frac{\mu\left(B_{r}(x)\right)}{\operatorname{vol}_{E}\left(B_{r}\left(\mathbb{R}^{n}\right)\right)}=1
$$

for every $x \in X$, where $B_{r}\left(\mathbb{R}^{n}\right)$ is the closed $r$-ball in the Euclidean space $\mathbb{R}^{n}$ and the $B_{r}(x)$ is the closed $r$-ball with the center $x \in X$.

In this chapter, the superscript of $n$ in the space $X^{n}$ means the mm-space $\left(X^{n}, d, \mu\right)$ satisfies $n$-dimensional condition.

Note that a closed smooth $n$-manifold $M^{n}(n \geq 3)$ admits a Riemannian metric with constant negative scalar curvature and a Riemannian metric of non-negative scalar curvature which is not identically zero, then by a conformal change of the metric we get a metric of positive scalar curvature according to Kazdan-Warner theorem [KW75]. Furthermore, if there is a scalar-flat Riemannian metric $g$ on $M^{n}$, but $g$ is not Ricci-flat metric, then $g$ can be deformed to a metric with positive scalar curvature according to Kazdan theorem [Kaz82, Theorem B] or by using Ricci-flow with an easy argument. Hence we will focus more on promoting the positive scalar curvature to positive $n$-volumic scalar curvature.

Definition 3.3 ( $n$-volumic scalar curvature). Assume $X^{n}=\left(X^{n}, d, \mu\right)$ is the compact mm-space and satisfies the $n$-dimensional condition, we call 
(i) the $n$-volumic scalar curvature of $X^{n}$ is positive, i.e. $S c^{\mathrm{vol}_{n}}\left(X^{n}\right)>0$, if there exists $r_{X^{n}}>0$ such that the measures of $\epsilon$-balls in $X^{n}$ are smaller than the volumes of $\epsilon$-balls in $\mathbb{R}^{n}$ for $0<\epsilon \leq r_{X^{n}}$.

(ii) the $n$-volumic scalar curvature of $X^{n}$ is bounded below by 0 , i.e. $S c^{\mathrm{vol}_{n}}\left(X^{n}\right) \geq 0$, if there exists $r_{X^{n}}>0$ such that the measures of $\epsilon$-balls in $X^{n}$ are not greater than the volumes of $\epsilon$-balls in $\mathbb{R}^{n}$ for $0<\epsilon \leq r_{X^{n}}$.

The $r_{X^{n}}$ is called scalar curvature radius (SC-radius) of $X^{n}$ for $S c^{\mathrm{vol}_{n}}\left(X^{n}\right) \geq 0$.

(iii) the $n$-volumic scalar curvature of $X^{n}$ is bounded below by $\kappa>0$, i.e. $S c^{\operatorname{vol}_{n}}\left(X^{n}\right) \geq \kappa>0$, if, for any $\gamma$ with $\gamma>\sqrt{\frac{2}{\kappa}}$, there exists $r_{X^{n}, \gamma}>0$ such that the measures of $\epsilon$-balls in $X^{n}$ are smaller than the volumes of $\epsilon$-balls in $S^{2}(\gamma) \times \mathbb{R}^{n-2}$ for $0<\epsilon \leq r_{X^{n}, \gamma}$.

We call

$$
r_{X^{n}}:=\inf _{\gamma>\sqrt{\frac{2}{\kappa}}} r_{X^{n}, \gamma}
$$

is the SC-radius of $X^{n}$ for $S c^{\mathrm{vol}_{n}}\left(X^{n}\right) \geq \kappa>0$.

In particular, we will focus on the case of

$$
\inf _{\gamma>\sqrt{\frac{2}{\kappa}}} r_{X^{n}, \gamma} \neq 0
$$

for stability.

If the mm-space $X^{n}$ is locally compact, then the definition of the $n$-volumic scalar curvature bounded below only modifies the definition of the $r_{X^{n}, \gamma}>0$ to a positive continuous function of $X^{n}$.

Two mm-spaces $\left(X^{n}, d, \mu\right)$ and $\left(X_{1}^{n}, d_{1}, \mu_{1}\right)$ are isometric if there exists a one-to-one map $f: X^{n} \rightarrow X_{1}^{n}$ such that $d_{1}(f(a), f(b))=d(a, b)$ for $a$ and $b$ are in $X^{n}$ and $f_{*} \mu=\mu_{1}$, where $f_{*} \mu$ is the push-forward measure, i.e. $f_{*} \mu(U)=\mu\left(f^{-1}(U)\right)$ for a measureable subset $U \subset X_{1}^{n}$. If $X^{n}$ satisfies $S c^{\mathrm{vol}_{n}}\left(X^{n}\right) \geq \kappa \geq 0$, then each mm-space $\left(X_{1}^{n}, d_{1}, \mu_{1}\right)$ that is isometric to $\left(X^{n}, d, \mu\right)$ also satisfies $S c^{\mathrm{vol}_{n}}\left(X_{1}^{n}\right) \geq \kappa \geq 0$. 
Proposition 3.4. Let $g$ be a $C^{2}$-smooth Riemannian metric on a closed oriented n-manifold $M^{n}$ with induced metric measure space $\left(M^{n}, d_{g}, d \mathrm{Vol}_{g}\right)$, then the scalar curvature of $g$ is positive, $S c_{g}>0$, if and only if $S c^{\mathrm{vol}_{n}}\left(M^{n}\right)>0$, and $S c_{g} \geq \kappa>0$ if and only if $S c^{\mathrm{vol}_{n}}\left(M^{n}\right) \geq \kappa>0$.

Proof. For a $C^{2}$-smooth Riemannian metric $g$, one has

$$
d \operatorname{Vol}_{g}\left(B_{r}(x)\right)=\operatorname{vol}_{E}\left(B_{r}\left(\mathbb{R}^{n}\right)\right)\left[1-\frac{S c_{g}(x)}{6(n+2)} r^{2}+O\left(r^{4}\right)\right]
$$

for $B_{r}(x) \subset M^{n}$ as $r \rightarrow 0$. Hence $\left(M^{n}, d_{g}, d \operatorname{Vol}_{g}\right)$ satisfies the $n$-dimensional condition.

If we have $S c_{g}>0$, then, since $M^{n}$ is compact, there exists $r_{M^{n}}>0$, so that $d \operatorname{Vol}_{g}\left(B_{r}(x)\right)<\operatorname{vol}_{E}\left(B_{r}\left(\mathbb{R}^{n}\right)\right)$ for all $0<r \leq r_{M^{n}}$. On the other hand, if there exists $r_{M^{n}}>0$ such that $d \operatorname{Vol}_{g}\left(B_{r}(x)\right)<\operatorname{vol}_{E}\left(B_{r}\left(\mathbb{R}^{n}\right)\right)$ for all $0<r \leq r_{M^{n}}$, then $S c_{g}$ must be greater than 0 .

If $S c^{\mathrm{vol}_{n}}\left(M^{n}\right) \geq \kappa>0$, then $S c_{g} \geq \kappa>0$. Otherwise, assume there exist small $\epsilon>0$ such that $S c_{g} \geq \kappa-\epsilon>0$. That means that there exists a point $x_{0}$ in $M^{n}$ such that $S c_{g}\left(x_{0}\right)=\kappa-\epsilon$, as $M^{n}$ is compact and the scalar curvature is a continuous function on $M^{n}$. Thus, we can find a small $r$-ball $B_{r}\left(x_{0}\right)$ such that the volume of $B_{r}\left(x_{0}\right)$ is greater than the volume of the $r$-ball in the $S^{2}(\gamma) \times \mathbb{R}^{n-2}$ for $\gamma=\sqrt{\frac{2}{\kappa-\frac{\epsilon}{2}}}$, which is a contradiction.

On the other hand, $S c_{g} \geq \kappa>0$ implies $S c^{\mathrm{vol}_{n}}\left(M^{n}\right) \geq \kappa>0$. Assume $S c_{g}\left(x_{1}\right)=\kappa$ for some $x_{1} \in M^{n}$, then there exists $r_{1}$ such that $d \operatorname{Vol}_{g}\left(B_{r_{1}}(x)\right) \leq d \operatorname{Vol}_{g}\left(B_{r_{1}}\left(x_{1}\right)\right)$ for $r_{1}$-balls in $M^{n}$ and

$$
d \operatorname{Vol}_{g}\left(B_{r}\left(x_{1}\right)\right)=\operatorname{vol}_{E}\left(B_{r}\left(\mathbb{R}^{n}\right)\right)\left[1-\frac{\kappa}{6(n+2)} r^{2}+O\left(r^{4}\right)\right]
$$

as $r \rightarrow 0$. Thus, for any $\gamma$ with $\gamma>\sqrt{\frac{2}{\kappa}}$, there exists $r_{M^{n}, \gamma}>0$ such that the measures of $\epsilon$-balls in $M^{n}$ are smaller than the volumes of $\epsilon$-balls in $S^{2}(\gamma) \times \mathbb{R}^{n-2}$ for $0<\epsilon \leq r_{M^{n}, \gamma}$, i.e. $S c^{\mathrm{vol}_{n}}\left(M^{n}\right) \geq \kappa>0$. 
Therefore, we have $S_{\frac{k}{n-1}}^{n}<_{\text {vol }} S^{2}(\gamma) \times \mathbb{R}^{n-2}$ for all $\gamma>\sqrt{\frac{2}{n \kappa}}$. Here $S_{\frac{k}{n-1}}^{n}$ is the Riemannian manifold $S^{n}$ with constant sectional curvature $\frac{\kappa}{n-1}$.

Remark 3.5. For a closed smooth Riemannian manifold $\left(M^{n}, g\right), S c^{\mathrm{vol}_{n}}\left(M^{n}\right) \geq 0$ implies $S c_{g} \geq 0$. Otherwise, there exist a point in $M^{n}$ such that the scalar curvature is negative, then the volume of small ball will be greater than the volume of the small ball in Euclidean space, which is a contradiction.

On the other hand, one can consider the case of the scalar-flat metric, i.e. $S c_{g} \equiv 0$. If $g$ is a strongly scalar-flat metric, meaning a metric with scalar curvature zero such that $M^{n}$ has no metric with positive scalar curvature, then $g$ is also Ricci flat according to Kazdan theorem above. Thus, we have

$$
d \operatorname{Vol}_{g}\left(B_{r}(x)\right)=\operatorname{vol}_{E}\left(B_{r}\left(\mathbb{R}^{n}\right)\right)\left[1-\frac{\|\operatorname{Rie}(x)\|_{g}^{2}}{120(n+2)(n+4)} r^{4}+O\left(r^{6}\right)\right]
$$

for $B_{r}(x) \subset M^{n}$ as $r \rightarrow 0$ [GV79, Theorem 3.3]. Here Rie is the Riemannian tensor. Therefore, if $g$ is a not flat metric, then $M^{n}<_{v o l} \mathbb{R}^{n}$. If $g$ is a flat metric, then $M^{n} \leq_{v o l} \mathbb{R}^{n}$. Thus $S c_{g} \geq 0$ implies $S c^{\text {vol }_{n}}\left(M^{n}\right) \geq 0$ for a strongly scalar-flat metric $g$.

However, $S c_{g} \geq 0$ may not imply $S c^{\mathrm{vol}_{n}}\left(M^{n}\right) \geq 0$. There are a lot of scalar-flat metrics but not strongly scalar flat metrics, i.e. $S c_{g} \equiv 0$ but not $R i c c_{g} \neq 0$. For instance, the product metric on $S^{2}(1) \times \Sigma$, where $\Sigma$ is a closed hyperbolic surface, is the scalar-flat metric, but not the Ricci-flat metric. For those metrics, we have

$$
d \operatorname{Vol}_{g}\left(B_{r}(x)\right)=\operatorname{vol}_{E}\left(B_{r}\left(\mathbb{R}^{n}\right)\right)\left[1+\frac{-3\|\operatorname{Rie}(x)\|_{g}^{2}+8\|\operatorname{Ricc}(x)\|_{g}^{2}}{360(n+2)(n+4)} r^{4}+O\left(r^{6}\right)\right]
$$

for $B_{r}(x) \subset M^{n}$ as $r \rightarrow 0\left[\overline{G V 79}\right.$, Theorem 3.3]. If 8\|Ricc $(x)\left\|_{g}^{2}>-3\right\| \operatorname{Rie}(x) \|_{g}^{2}$ for some point, then $S c_{g} \geq 0$ does not imply $S c^{\mathrm{vol}_{n}}\left(M^{n}\right) \geq 0$.

Theorem 3.6. Assume that the mm-space $\left(X^{n}, d, \mu\right)$ satisfies $n$-dimensional condition and the curvature-dimension condition $C D(\kappa, n)$ for $\kappa \geq 0$ and $n \geq 2$, then $\left(X^{n}, d, \mu\right)$ satisfies $\operatorname{Sc}^{\mathrm{vol}_{n}}\left(X^{n}\right) \geq n \kappa$. 
Proof. In fact, one only needs the generalized Bishop-Gromov volume growth inequality, which is implied by the curvature-dimension of $X^{n}$ [Stu06b, Theorem 2.3].

(i) If $\kappa=0$, then

$$
\frac{\mu\left(B_{r}(x)\right)}{\mu\left(B_{R}(x)\right)} \geq\left(\frac{r}{R}\right)^{n}
$$

for all $0<r<R$. That is

$$
\frac{\mu\left(B_{r}(x)\right)}{\operatorname{vol}_{E}\left(B_{r}\left(\mathbb{R}^{n}\right)\right)}=\frac{\mu\left(B_{r}(x)\right)}{\alpha(n) r^{n}} \geq \frac{\mu\left(B_{R}(x)\right)}{\alpha(n) R^{n}}=\frac{\mu\left(B_{R}(x)\right)}{\operatorname{vol}_{E}\left(B_{R}\left(\mathbb{R}^{n}\right)\right)}
$$

where $\alpha(n)=\frac{\operatorname{vol}_{E}\left(B_{r}\left(\mathbb{R}^{n}\right)\right)}{r^{n}}$. Combining the $n$-dimensional condition,

$$
\lim _{r \rightarrow 0} \frac{\mu\left(B_{r}(x)\right)}{\operatorname{vol}_{E}\left(B_{r}\left(\mathbb{R}^{n}\right)\right)}=1
$$

that implies $S c^{\mathrm{vol}_{n}}(X) \geq 0$.

(ii) If $\kappa>0$, then

$$
\frac{\mu\left(B_{r}(x)\right)}{\mu\left(B_{R}(x)\right)} \geq \frac{\int_{0}^{r}\left[\sin \left(t \sqrt{\frac{\kappa}{(n-1)}}\right)\right]^{n-1} d t}{\int_{0}^{R}\left[\sin \left(t \sqrt{\frac{\kappa}{(n-1)}}\right)\right]^{n-1} d t}
$$

for all $0<r \leq R \leq \pi \sqrt{\frac{(n-1)}{\kappa}}$.

Since the scalar curvature of the product manifold $S^{2}(\gamma) \times \mathbb{R}^{n-2}$ is $n \kappa$, where $\gamma=\sqrt{\frac{2}{n \kappa}}$, then there exists $C_{1}, C_{2}>0$ such that

$$
1-\frac{n \kappa}{6(n+2)} r_{1}^{2}-C_{2} r_{1}^{4} \leq \operatorname{vol}_{S \times E}\left(B_{r_{1}}(y)\right):=\frac{\operatorname{vol}_{S \times E}\left(B_{r_{1}}(y)\right)}{\operatorname{vol}_{E}\left(B_{r_{1}}\left(\mathbb{R}^{n}\right)\right)} \leq 1-\frac{n \kappa}{6(n+2)} r_{1}^{2}+C_{2} r_{1}^{4},
$$

for $y \in S^{2}(\gamma) \times \mathbb{R}^{n-2}$ and $r_{1} \leq C_{1}$, where $C_{1}, C_{2}$ are decided by the product manifold $S^{2}(\gamma) \times \mathbb{R}^{n-2}$.

Let

$$
\mu\left(\widetilde{B_{r}(x)}\right):=\frac{\mu\left(B_{r}(x)\right)}{\operatorname{vol}_{E}\left(B_{r}\left(\mathbb{R}^{n}\right)\right)}
$$


and

$$
f(r):=\frac{\int_{0}^{r}\left[\sin \left(t \sqrt{\frac{\kappa}{(n-1)}}\right)\right]^{n-1} d t}{\operatorname{vol}_{E}\left(B_{r}\left(\mathbb{R}^{n}\right)\right)},
$$

then the generalized Bishop-Gromov inequality can be re-formulated as

$$
\mu\left(\widetilde{B_{R}(x)}\right) \leq \mu\left(\widetilde{B_{r}(x)}\right) \frac{f(R)}{f(r)}
$$

for all $0<r<R \leq \pi \sqrt{\frac{(n-1)}{\kappa}}$. The asymptotic expansion of $f(r)$ is

$$
f(r)=\frac{\frac{1}{n} r^{n}\left[\frac{\kappa}{(n-1)}\right]^{\frac{(n-1)}{2}}-\frac{(n-1)}{6(n+2)} r^{n+2}\left[\frac{\kappa}{(n-1)}\right]^{\frac{n+1}{2}}+O\left(r^{n+4}\right)}{\operatorname{vol}_{E}\left(B_{r}\left(\mathbb{R}^{n}\right)\right)}
$$

as $r \rightarrow 0$. Thus, the asymptotic expansion of $\frac{f(R)}{f(r)}$ is

$$
\frac{f(R)}{f(r)}=\frac{1-\frac{n \kappa}{6(n+2)} R^{2}+O\left(R^{4}\right)}{1-\frac{n \kappa}{6(n+2)} r^{2}+O\left(r^{4}\right)}
$$

as $R \rightarrow 0, r \rightarrow 0$. The $n$-dimensional condition, $\lim _{r \rightarrow 0} \mu\left(\widetilde{B_{r}(x)}\right)=1$, implies that

$$
\mu\left(\widetilde{B_{R}(x)}\right) \leq 1-\frac{n \kappa}{6(n+2)} R^{2}+O\left(R^{4}\right)
$$

as $R \rightarrow 0$. Therefore, for any $\kappa^{\prime}$ with $0<\kappa^{\prime}<\kappa$, there exists $\epsilon_{\kappa^{\prime}}>0$ such that for any $0<R \leq \epsilon_{K^{\prime}}$, we have

$$
\left.\mu\left(\widetilde{B_{R}(x)}\right)<\operatorname{vol}_{S \times E} \widetilde{\left(B_{R}\right.}(y)\right)
$$

where

$$
\left.\operatorname{vol}_{S \times E} \widetilde{\left(B_{R}\right.}(y)\right)=\frac{\operatorname{vol}_{S \times E}\left(B_{R}(y)\right)}{\operatorname{vol}_{E}\left(B_{R}\left(\mathbb{R}^{n}\right)\right)}
$$

is defined as before, the balls $B_{R}(y)$ are in $S^{2}(\gamma) \times \mathbb{R}^{n-2}$ and $\gamma=\sqrt{\frac{2}{n \kappa^{\prime}}}$. That is

$$
X^{n}<_{v o l} S^{2}(\gamma) \times \mathbb{R}^{n-2}
$$

for all $\gamma>\sqrt{\frac{2}{n \kappa}}$, i.e. $S c^{\mathrm{vol}_{n}}\left(X^{n}\right) \geq n \kappa$.

In fact, one has the classical Bishop inequality by adding the $n$-dimensional condition to the generalized Bishop-Gromov volume growth inequality. It means that 
- if $\kappa=0, \mu\left(B_{R}(x)\right) \leq \operatorname{vol}_{E}\left(B_{R}\left(\mathbb{R}^{n}\right)\right)$ for all $R>0$.

- if $\kappa>0$,

$$
\mu\left(B_{R}(x)\right) \leq \operatorname{vol}_{S^{n}}\left(B_{R}\left(S_{\frac{\kappa}{n-1}}^{n}\right)\right)
$$

for

$$
0<R \leq \pi \sqrt{\frac{(n-1)}{\kappa}} .
$$

In other words, if $\kappa=0, X^{n} \leq_{v o l} \mathbb{R}^{n}$. If $\kappa>0, X^{n} \leq_{v o l} S_{\frac{\kappa}{n-1}}^{n}$. We have

$$
S_{\frac{K}{n-1}}^{n}<_{v o l} S^{2}(\gamma) \times \mathbb{R}^{n-2}
$$

for all $\gamma>\sqrt{\frac{2}{n \kappa}}$. Then $X^{n}<_{\text {vol }} S^{2}(\gamma) \times \mathbb{R}^{n-2}$ for all $\gamma>\sqrt{\frac{2}{n \kappa}}$.

Thus, we also get $S c^{\mathrm{vol}_{n}}\left(X^{n}\right) \geq n \kappa$.

Remark 3.7. Hence the mm-space $\left(X^{n}, d, \mu\right)$ with $S c^{\mathrm{vol}_{n}}\left(X^{n}\right) \geq n \kappa \geq 0$ includes the mmspaces that satisfies $n$-dimensional condition and the generalized Bishop-Gromov volume growth inequality as stated in the proof, e.g., the mm-spaces with the Riemannian curvature condition $R C D(\kappa, n)$ [AGS14] or with the measure concentration property $\operatorname{MCP}(\kappa, n)$ [Oht07].

In particular, De Philippis and Gigli defined the non-collapsed $\operatorname{RCD}(\kappa, n)$ space and showed that assume $\left(X, d, \mathcal{H}^{n}\right)$ is a non-collapsed $\operatorname{RCD}(0, n)$ and all points in $X$ is regular (i.e., all tangent cones are isometric to $\left.\mathbb{R}^{n}\right)$, then $\left(X, d, \mathcal{H}^{n}\right)$ satisfies the $n$-dimensional condition [DPG18, Corollary 1.7]. Here $\mathcal{H}^{n}$ is $n$-dimensional Hausdorff measure. Thus, regular non-collapsed $R C D(\kappa, n)$ space $\left(X, d, \mathcal{H}^{n}\right)$ satisfies $S c^{\mathrm{vol}_{n}}(X) \geq 0$.

Question 3.8. Let $A l^{n}(1)$ be an orientable compact $n$-dimensional Alexandrov space with curvature $\geq 1$, then do all continuous maps $\phi$ from $A l^{n}(1)$ to the sphere $S^{n}$ with standard metric of non-zero degree satisfy $\operatorname{Lip}(\phi) \geq C(n)$ ? Here Lip $(\phi)$ is the Lipschitz constant of $\phi$, $\phi$ maps the boundary of $A l^{n}(1)$ to a point in $S^{n}$ and $C(n)$ is a constant depending only on the dimension $n$. 
Question 3.9. Assume the compact mm-space $(X, d, \mu)$ satisfies the curvature-dimension condition $C D(n-1, n), n$-dimensional condition and the covering dimension is also $n$, then do all continuous maps $\phi$ from $(X, d, \mu)$ to the sphere $S^{n}$ with standard metric, where $\phi$ is non-trivial in the homotopy class of maps, satisfy $\operatorname{Lip}(\phi) \geq C_{1}(n)$, where $C_{1}(n)$ is a constant only depends $n$ ?

Remark 3.10. The questions above are inspired by Gromov's spherical Lipschitz bounded theorem in [Gro18, Section 3] and the results above. The finite covering dimension is equal to the cohomological dimension over integer ring $\mathbb{Z}$ for the compact metric space according to the Alexandrov theorem. The best constant of $C(n)$ and $C_{1}(n)$ would be 1 if both questions have positive answers.

Proposition 3.11 (Quadratic Scaling). Assume the compact mm-space $\left(X^{n}, d, \mu\right)$ satisfies $S c^{\mathrm{vol}_{n}}\left(X^{n}\right) \geq \kappa>0$, then $S c^{\mathrm{vol}_{n}}\left(\lambda X^{n}\right) \geq \lambda^{-2} \kappa>0$ and $r_{\lambda X^{n}}=\lambda r_{X^{n}}$ for all $\lambda>0$, where $\lambda X^{n}:=\left(X^{n}, \lambda \cdot d, \lambda^{n} \cdot \mu\right)$.

Proof. First, we will show that the $n$-dimensional condition is stable under scaling. Let $d^{\prime}:=\lambda \cdot d, \mu^{\prime}:=\lambda^{n} \cdot \mu, B_{r}^{\prime}(x)$ be a $r$-ball in the $\left(X^{n}, d^{\prime}\right)$, and $B_{r}(x)$ be a $r$-ball in the $\left(X^{n}, d\right)$, then $B_{r}^{\prime}(x)=B_{\frac{r}{\lambda}}(x)$ as the subset in the $X^{n}$. One has

$$
\lim _{r \rightarrow 0} \frac{\mu^{\prime}\left(B_{r}^{\prime}(x)\right)}{\operatorname{vol}_{E}\left(B_{r}\left(\mathbb{R}^{n}\right)\right)}=\lim _{r \rightarrow 0} \frac{\mu^{\prime}\left(B_{\frac{r}{\lambda}}(x)\right)}{\operatorname{vol}_{E}\left(B_{r}\left(\mathbb{R}^{n}\right)\right)}=\lim _{r \rightarrow 0} \frac{\lambda^{n} \cdot \mu\left(B_{\frac{r}{\lambda}}(x)\right)}{\lambda^{n} \cdot \operatorname{vol}_{E}\left(B_{\frac{r}{\lambda}}\left(\mathbb{R}^{n}\right)\right)}=1,
$$

then $\lambda X^{n}$ satisfies the $n$-dimensional condition.

Since $\lambda \cdot\left(S^{2}(\gamma) \times \mathbb{R}^{n-2}\right)=\lambda \cdot S^{2}(\gamma) \times \lambda \cdot \mathbb{R}^{n-2}=S^{2}(\lambda \gamma) \times \lambda \cdot \mathbb{R}^{n-2}$, we have

$$
\lambda \cdot X^{n}<_{\text {vol }} \lambda \cdot\left(S^{2}(\gamma) \times \mathbb{R}^{n-2}\right)=S^{2}(\lambda \gamma) \times \lambda \cdot \mathbb{R}^{n-2}
$$

for all $\lambda \gamma>\sqrt{\frac{2}{\lambda \kappa}}$ and $0<\epsilon \leq \lambda r_{X^{n}}$. That means $S c^{\mathrm{vol}_{n}}\left(\lambda X^{n}\right) \geq \lambda^{-2} \kappa>0$ and $r_{\lambda \cdot X^{n}}=$ $\lambda r_{X^{n}}$

We also have $S c^{\mathrm{vol}_{n}}\left(\lambda X^{n}\right) \geq 0(>0)$, if $S c^{\mathrm{vol}_{n}}\left(X^{n}\right) \geq 0(>0)$. 
Remark 3.12. Since the $n$-dimensional condition and definition of $n$-volumic scalar curvature is locally defined, we have the following construction.

- Global to local: Let the locally compact mm-space $\left(X^{n}, d, \mu\right)$ satisfy $S c^{\mathrm{vol}_{n}}\left(X^{n}\right) \geq \kappa \geq 0$ and $Y^{n} \subset X$ be an open subset. Then, if $\left(Y^{n}, d_{Y}\right)$ is a complete length space, $\left(Y^{n}, d_{Y}, \mu\left\llcorner_{Y}\right)\right.$ satisfies $S c^{\mathrm{vol}_{n}}\left(Y^{n}\right) \geq \kappa \geq 0$ and $r_{Y^{n}}=r_{X^{n}}$. Where $d_{Y}$ is the induced metric of $d$ and $\mu\left\llcorner_{Y}\right.$ is the restriction operator, namely, $\mu\left\llcorner_{Y}(A):=\mu\left(Y^{n} \cap A\right)\right.$ for $A \subset X^{n}$.

- Local to global: Let $\left\{Y_{i}^{n}\right\}_{i \in I}$ be a finite open cover of a locally compact mm-space $\left(X^{n}, d, \mu\right)$. Assume that $\left(Y_{i}^{n}, d_{Y_{i}}\right)$ is a complete length space and $\left(Y_{i}^{n}, d_{Y_{i}}, \mu\left\llcorner_{Y_{i}}\right)\right.$ satisfies $S c^{\operatorname{vol}_{n}}\left(Y_{i}^{n}\right) \geq \kappa \geq 0$, then $\left(X^{n}, d, \mu\right)$ satisfies $S c^{\operatorname{vol}_{n}}\left(X^{n}\right) \geq \kappa \geq 0$ and $r_{X^{n}}$ can be chosen as a partition of unity of the functions $\left\{r_{Y_{i}^{n}}\right\}_{i \in I}$.

Question 3.13. Assume that $S c^{\mathrm{vol}_{n_{1}}}\left(X_{1}^{n_{1}}\right) \geq \kappa_{1}(\geq 0)$ for the compact mm-space $\left(X_{1}^{n_{1}}, d_{1}, \mu_{1}\right)$ and $S c^{\mathrm{vol}_{n_{2}}}\left(X_{2}^{n_{2}}\right) \geq \kappa_{2}(\geq 0)$ for the compact mm-space $\left(X_{2}^{n_{2}}, d_{2}, \mu_{2}\right)$, then do we have

$$
S c^{\mathrm{vol}_{n_{1}+n_{2}}}\left(X_{1}^{n_{1}} \times X_{2}^{n_{2}}\right) \geq \kappa_{1}+\kappa_{2}, \quad r_{X_{1}^{n} \times X_{2}^{n}}=\min \left\{r_{X_{1}^{n}}, r_{X_{2}^{n}}\right\}
$$

for $\left(X_{1}^{n_{1}} \times X_{2}^{n_{2}}, d_{3}, \mu_{3}\right)$ ? Here $X_{1}^{n_{1}} \times X_{2}^{n_{2}}$ is endowed with the measure $\mu_{3}:=\mu_{1} \otimes \mu_{2}$ and with the Pythagorean product metric $d_{3}:=\sqrt{d_{1}^{2}+d_{2}^{2}}$.

\subsection{SmGH-Convergence}

Let $\left\{\mu_{n}\right\}_{n \in \mathbb{N}}$ and $\mu$ be Borel measures on the space $X$, then the sequence $\left\{\mu_{n}\right\}_{n \in \mathbb{N}}$ is said to converge strongly (also called setwise convergence in other literature ) to a limit $\mu$ if

$$
\lim _{n \rightarrow \infty} \mu_{n}(\mathcal{A})=\mu(\mathcal{A})
$$

for every $\mathcal{A}$ in the Borel $\sigma$-algebra.

A map $f: X \rightarrow Y$ is called an $\epsilon$-isometry between compact metric spaces $X$ and $Y$, if

$$
\left|d_{X}(a, b)-d_{Y}(f(a), f(b))\right| \leq \epsilon
$$


for all $a, b \in X$ and it is almost surjective, i.e. for every $y \in Y$, there exists an $x \in X$ such that $d_{Y}(f(x), y) \leq \epsilon$.

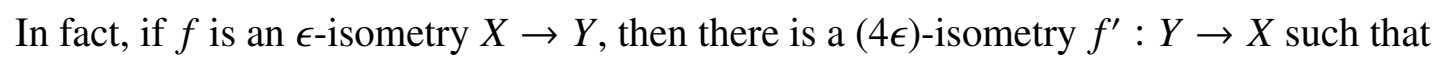
for all $x \in X, y \in Y, d_{X}\left(f^{\prime} \circ f(x), x\right) \leq 3 \epsilon, d_{Y}\left(f \circ f^{\prime}(y), y\right) \leq \epsilon$.

Definition 3.14 (smGH-convergence). Let $\left(X_{i}, d_{i}, \mu_{i}\right)_{i \in \mathbb{N}}$ and $(X, d, \mu)$ be compact mmspaces. $X_{i}$ converges to $X$ in the strongly measured Gromov-Hausdorff topology (smGHconvergence) if there are measurable $\epsilon_{i}$-isometries $f_{i}: X_{i} \rightarrow X$ such that $\epsilon_{i} \rightarrow 0$ and $f_{i *} \mu_{i} \rightarrow \mu$ in the strong topology of measures as $i \rightarrow \infty$.

If the spaces $\left(X_{i}, d_{n}, \mu_{i}, p_{i}\right)_{i \in \mathbb{N}}$ and $(X, d, \mu, p)$ are locally compact pointed mm-spaces, it is said that $X_{i}$ converges to $X$ in the pointed strongly measured Gromov-Hausdorff topology (psmGH-convergence) if there are sequences $r_{i} \rightarrow \infty, \epsilon_{i} \rightarrow 0$, and measurable pointed $\epsilon_{i}$-isometries $f_{i}: B_{r_{i}}\left(p_{i}\right) \rightarrow B_{r_{i}}(p)$, such that $f_{i *} \mu_{i} \rightarrow \mu$, where the convergence is strong convergence.

Remark 3.15. Let $\left(X_{i}, d_{i}, \mu_{i}\right)_{i \in \mathbb{N}}$ converge to $(X, d, \mu)$ in the measured Gromov-Hausdorff topology, then there are measurable $\epsilon_{i}$-isometries $f_{i}: X_{i} \rightarrow X$ such that $f_{i *} \mu_{i}$ weakly converges to $\mu$. If there is a Borel measure $v$ on $X$ such that $\sup f_{i *} \mu_{i} \leq v$, i.e.

$$
\sup _{i} f_{i *} \mu_{i}(\mathcal{A}) \leq v(\mathcal{A})
$$

for every $\mathcal{A}$ in the Borel $\sigma$-algebra on $X$, then $X_{i}$ smGH-converges to $X$ (See, [Las97, Lemma 4.1]).

Remark 3.16. The $n$-dimensional condition is not preserved by the measured GromovHausdorff convergence as the following example shows. Let $\left\{a_{i} S^{2}:=\left(S^{2}, a_{i} d_{S}\right)\right\}\left(a_{i} \in\right.$ $(0,1))$ be a sequence of space, then the limit of $a_{i} S^{2}$ under the measured Gromov-Hausdorff convergence is a point when $a_{i}$ goes to 0 . The limit exists as the Ricci curvature of $a_{i} S^{2}$ is bounded below by 1 . 
Remark 3.17. The $n$-dimensional condition is not preserved by the smGH-convergence since the limits of

$$
\lim _{r \rightarrow 0} \lim _{i \rightarrow \infty} \frac{\mu_{i}\left(B_{r}(x)\right)}{\operatorname{vol}_{E}\left(B_{r}\left(\mathbb{R}^{n}\right)\right)}
$$

may not be commutative for some mm-spaces $\left(X, d, \mu_{i}\right)$. Assume the total variation distance of the measures goes to 0 as $i \rightarrow \infty$, i.e.

$$
d_{T V}\left(\mu_{i}, \mu\right):=\sup _{\mathcal{A}}\left|\mu_{i}(\mathcal{A})-\mu(\mathcal{A})\right| \rightarrow 0
$$

where $\mathcal{A}$ runs over the Borel $\sigma$-algebra of $X$, then the limits are commutative.

One can define the total variation Gromov-Hausdorff convergence (tvGH-convergence) for mm-spaces by replacing the the strong topology with the topology induced by the total variation distance in definition of smGH-convergence. Then tvGH-convergence implies smGH-convergence and the $n$-dimensional condition is preserved by tvGH-convergence.

Theorem 3.18 (Stability). If compact mm-spaces $\left(X_{i}^{n}, d_{i}, \mu_{i}\right)$ with $S c^{\mathrm{vol}_{n}}\left(X_{i}^{n}\right) \geq \kappa \geq 0$, $S C$-radius $r_{X_{i}^{n}} \geq R>0$, and $\left(X_{i}^{n}, d_{i}, \mu_{i}\right)$ smGH-converge to the compact mm-space $\left(X^{n}, d, \mu\right)$ with $n$-dimensional condition, then $X^{n}$ also satisfies $S c^{\mathrm{vol}_{n}}\left(X^{n}\right) \geq \kappa$ and the $S C$-radius $r_{X^{n}} \geq R$

Proof. Fix a $x \in X^{n}$ and let $B_{r}(x)$ be the small $r$-ball on $X^{n}$ where $r<R$, then there exists $x_{i} \in X_{i}^{n}$ such that $f_{i}^{-1}\left(B_{r}(x)\right) \subset B_{r+4 \epsilon_{i}}\left(x_{i}\right)$ where $B_{r+4 \epsilon_{i}}\left(x_{i}\right) \subset X_{i}^{n}$ and $r+4 \epsilon_{i} \leq R$. Thus, $f_{i *} \mu_{i}\left(B_{r}(x)\right) \leq \mu_{i}\left(B_{r+4 \epsilon_{i}}\left(x_{i}\right)\right)$.

- For $\kappa=0$, since $S c^{\operatorname{vol}_{n}}\left(X_{i}^{n}\right) \geq 0$ and SC-radius $\geq R>0$, then $\mu_{i}\left(B_{r}\left(x_{i}\right)\right) \leq \operatorname{vol}_{E}\left(B_{r}\left(\mathbb{R}^{n}\right)\right)$ for all $0<r \leq R$ and all $i$. Therefore,

$$
f_{i *} \mu_{i}\left(B_{r}(x)\right)<\mu_{i}\left(B_{r+4 \epsilon_{i}}\left(x_{i}\right)\right) \leq \operatorname{vol}_{E}\left(B_{r+4 \epsilon_{i}}\left(\mathbb{R}^{n}\right)\right)
$$

for $r+4 \epsilon_{i} \leq R$. Then $\mu\left(B_{r}(x)\right) \leq \operatorname{vol}_{E}\left(B_{r}\left(\mathbb{R}^{n}\right)\right)$, since $\epsilon_{i}$ that is not related to $r$ can be arbitrarily small. 
- $\quad$ For $\kappa>0$, we have $\mu_{i}\left(B_{r}\left(x_{i}\right)\right)<\operatorname{vol}_{S \times E}\left(B_{r}\left(S^{2}(\gamma) \times \mathbb{R}^{n-2}\right)\right)$ for all $0<r \leq R$, all $i$, and $\gamma>\sqrt{\frac{2}{\kappa}}$. Thus,

$$
f_{i *} \mu_{i}\left(B_{r}(x)\right)<\mu_{i}\left(B_{r+4 \epsilon_{i}}\left(x_{i}\right)\right)<\operatorname{vol}_{S \times E}\left(B_{r+4 \epsilon_{i}}\left(S^{2}(\gamma) \times \mathbb{R}^{n-2}\right)\right)
$$

for $r+4 \epsilon_{i} \leq R$. Since $\epsilon_{i}$ that is not related to $r$ can be arbitrarily small, then

$$
\mu\left(B_{r}(x)\right) \leq \operatorname{vol}_{S \times E}\left(B_{r}\left(S^{2}(\gamma) \times \mathbb{R}^{n-2}\right)\right)
$$

for $\gamma>\sqrt{\frac{2}{\kappa}}$. Thus,

$$
\mu\left(B_{r}(x)\right)<\operatorname{vol}_{S \times E}\left(B_{r}\left(S^{2}\left(\sqrt{\frac{2}{\kappa+\epsilon^{\prime}}}\right) \times \mathbb{R}^{n-2}\right)\right),
$$

where $0<\epsilon^{\prime}$ is independence on $r$ and $\epsilon^{\prime}$ can as small as we want. Therefore, we have $S c^{\mathrm{vol}_{n}}\left(X^{n}\right) \geq \kappa$.

Definition 3.19 (Tangent space). The mm-space $\left(Y, d_{Y}, \mu_{Y}, o\right)$ is a tangent space of $\left(X^{n}, d, \mu\right)$ at $p \in X^{n}$ if there exists a sequence $\lambda_{i} \rightarrow \infty$ such that $\left(X^{n}, \lambda_{i} \cdot d, \lambda_{i}^{n} \cdot \mu, p\right)$ psmGH-converges to $\left(Y, d_{Y}, \mu_{Y}, o\right)$ as $\lambda_{i} \rightarrow \infty$.

Therefore, $\left(Y, d_{Y}, \mu_{Y}, o\right)$ also satisfies the $n$-dimensional condition and can be written as $Y^{n}$.

Corollary 3.20. Assume the compact mm-space $\left(X^{n}, d, \mu\right)$ with $S c^{\mathrm{vol}_{n}}\left(X^{n}\right) \geq \kappa \geq 0$ and the tangent space $\left(Y^{n}, d_{Y}, \mu_{Y}, o\right)$ of $X^{n}$ exists at the point $p$, then $\left(Y^{n}, d_{Y}, \mu_{Y}, o\right)$ satisfies $S c^{\mathrm{vol}_{n}}\left(Y^{n}\right) \geq 0$ and the $S C$-radius $\geq r_{X^{n}}$.

Proof. Since the $n$-volumic scalar curvature has the quadratic scaling property, i.e. $S c^{\operatorname{vol}_{n}}\left(\lambda X^{n}\right) \geq$ $\lambda^{-2} \kappa \geq 0$ and $r_{\lambda X^{n}}=\lambda r_{X^{n}}$ for all $\lambda>0$, where $\lambda X^{n}:=\left(X^{n}, \lambda \cdot d, \lambda^{n} \cdot \mu\right)$, then $S c^{\operatorname{vol}_{n}}\left(Y^{n}\right) \geq 0$ is implied by the stability theorem. 
The mm-spaces with $S c^{\mathrm{vol}_{n}} \geq 0$ includes some of the Finsler manifolds, for instance, $\mathbb{R}^{n}$ equipped with any norm and with the Lebesgue measure satisfies $S c^{\mathrm{vol}_{n}} \geq 0$ and any smooth compact Finsler manifold is a $C D(\kappa, n)$ space for appropriate finite $\kappa$ and $n$ [Oht09]. It is well-known that Gigli's infinitesimally Hilbertian [Gig15] can be seen as the Riemannian condition in $R C D(\kappa, n)$ space. Thus, infinitesimally Hilbertian can also be used as a Riemannian condition in the mm-spaces with $S c^{\mathrm{vol}_{n}} \geq 0$.

Definition 3.21 $\left(R S C(\kappa, n)\right.$ space). The compact mm-space $\left(X^{n}, d, \mu\right)$ with the $n$-dimensional condition is a Riemannian $n$-volumic scalar curvature $\geq \kappa$ space $(\operatorname{RSC}(\kappa, n)$ space $)$ if it is infinitesimally Hilbertian and satisfies the $S c^{\mathrm{vol}_{n}}\left(X^{n}\right) \geq \kappa \geq 0$.

Note that any finite dimensional Alexandrov spaces with curvature bounded below are infinitesimally Hilbertian. Then

$$
A l^{n}(\kappa) \Rightarrow R C D((n-1) \kappa, n) \Rightarrow R S C((n(n-1) \kappa, n)
$$

on $\left(X^{n}, d, \mathcal{H}^{n}\right)$, where the measure $\mathcal{H}^{n}$ is the $n$-dimensional Hausdorff measure that satisfies the $n$-dimensional condition.

Question 3.22. Are RSC $(\kappa, n)$ spaces stable under tvGH-convergence?

Remark 3.23 (Convergence of compact mm-spaces). For the compact metric measure spaces with probability measures, one can consider mGH-convergence, Gromov-Prokhorov convergence, Gromov-Hausdorff-Prokhorov convergence, Gromov-Wasserstein convergence, Gromov-Hausdorff-Wasserstein convergence, Gromov’s ㅁ-convergence, Sturm’s D-convergence [Vil09, Section 27], and Gromov-Hausdorff-vague convergence [ALW16]. smGH-convergence implies those convergences for compact metric measure spaces with probability measures, since the measures converge strongly in smGH-convergence and converge weakly in other situations.

Note that mm-spaces with infinitesimally Hilbertian are generally not stable under mGH-convergence [Gig15]. It is not clear if the infinitesimally Hilbertian are preserved under smGH-convergence or tvGH-convergence. 



\section{Weighted Scalar Curvature $S c_{\alpha, \beta}$}

Let the smooth metric measure space $\left(M^{n}, g, e^{-f} d \mathrm{Vol}_{g}\right.$ ) (also known as the weighted Riemannian manifold in some references), where $f$ is a $C^{2}$-function on $M^{n}, g$ is a $C^{2}$ Riemannian metric and $n \geq 2$, satisfies the curvature-dimension condition $C D(\kappa, n)$ for $\kappa \geq 0$, then $M^{n}$ also satisfies $S c^{\text {vol }_{n}}\left(M^{n}\right) \geq n \kappa$.

Motivated by the importance of the Ricci Bakry-Emery curvature, i.e.

$$
\operatorname{Ric}_{f}^{M}=\operatorname{Ricc}+\operatorname{Hess}(f),
$$

the weighted sectional curvature of smooth mm-space was proposed and discussed in [Wyl15]. On the other hand, Perelman defined and used the P-scalar curvature in his $\mathcal{F}$-functional in [Per02, Section 1]. Inspired by the P-scalar curvature, i.e.

$$
S c_{g}+2 \triangle_{g} f-\left\|\nabla_{g} f\right\|_{g}^{2}
$$

we propose another weighted scalar curvature on the smooth mm-space.

This chapter comes also from my published paper [Den21a].

Definition 4.1 (Weighted scalar curvature $S c_{\alpha, \beta}$ ). The weighted scalar curvature $S c_{\alpha, \beta}$ on the smooth mm-space $\left(M^{n}, g, e^{-f} d \mathrm{Vol}_{g}\right)$ is defined by

$$
S c_{\alpha, \beta}:=S c_{g}+\alpha \triangle_{g} f-\beta\left\|\nabla_{g} f\right\|_{g}^{2}
$$

Note that the Laplacian $\triangle_{g}$ here is the trace of the Hessian and $S c^{\text {vol }_{n}}\left(M^{n}\right) \geq \kappa \geq 0$ is equivalent to $S c_{\alpha, \beta} \geq \kappa \geq 0$ for $\alpha=3$ and $\beta=3$ (See [Qia97, Theorem 8] or the proof of Corollary 4.10 below). 
Example 4.2. (1). For $\alpha=\frac{2(n-1)}{n}$ and $\beta=\frac{(n-1)(n-2)}{n^{2}}$, the $S c_{\frac{2(n-1)}{n}, \frac{(n-1)(n-2)}{n^{2}}}$ is the Chang-GurskyYang's conformally invariant scalar curvature for the smooth mm-space [CGY06]. That means for a $C^{2}$-smooth function $w$ on $M^{n}$, one has

$$
S c_{\frac{2(n-1)}{n}, \frac{(n-1)(n-2)}{n^{2}}}\left(e^{2 w} g\right)=e^{-2 w} S c_{\frac{2(n-1)}{n}, \frac{(n-1)(n-2)}{n^{2}}}(g) .
$$

(2). For $\alpha=2$ and $\beta=\frac{m+1}{m}$, where $m \in \mathbb{N} \cup\{0, \infty\}$, the $S c_{2, \frac{m+1}{m}}$ is Case's weighted scalar curvature and Case also defined and studied the weighted Yamabe constants in [Cas13]. Case's weighted scalar curvature is the classical scalar curvature if $m=0$. If $m=\infty$, then it is Perelman's P-scalar curvature.

Note that the results in this chapter are new for those examples.

\subsection{Spin manifold and $S c_{\alpha, \beta}>0$}

For an orientable closed surface with density $\left(\Sigma, g, e^{-f} d \mathrm{Vol}_{g}\right)$ with $S c_{\alpha, \beta}>0$ and $\beta \geq 0$, then the inequality,

$$
\begin{array}{r}
0<\int_{\Sigma} S c_{\alpha, \beta} d \operatorname{Vol}_{g}=\int_{\Sigma}\left(S c_{g}+\alpha \Delta_{g} f-\beta\left\|\nabla_{g} f\right\|_{g}^{2}\right) d \operatorname{Vol}_{g} \\
=4 \pi \chi(\Sigma)-\beta \int_{\Sigma}\left\|\nabla_{g} f\right\|_{g}^{2} d \operatorname{Vol}_{g}
\end{array}
$$

implies that $\chi(\Sigma)>0$. Thus, $\Sigma$ is a 2 -sphere.

The following proposition of vanishing harmonic spinors is owed to Perelman essentially and the proof is borrowed from [AC17, Proposition 1].

Proposition 4.3 (Vanishing harmonic spinors). Assume the smooth mm-space $\left(M^{n}, g, e^{-f} d \mathrm{Vol}_{g}\right)$ is closed and spin. If $\alpha \in \mathbb{R}, \beta \geq \frac{|\alpha|^{2}}{4}$ and $S c_{\alpha, \beta}>0$, then the harmonic spinor of $M^{n}$ vanishes. 
Proof. Let $\psi$ be a harmonic spinor, though the Schroedinger-Lichnerowicz-Weitzenboeck formula

$$
\mathbb{D}^{2}=\nabla^{*} \nabla+\frac{1}{4} S c_{g}
$$

one has

$$
\begin{array}{r}
0=\int_{M}\left[\left\|\nabla_{g} \psi\right\|_{g}^{2}+\frac{1}{4}\left(S c_{\alpha, \beta}-\alpha \Delta_{g} f+\beta\left\|\nabla_{g} f\right\|_{g}^{2}\right)\|\psi\|_{g}^{2}\right] d \operatorname{Vol}_{g} \\
=\int_{M}\left[\left\|\nabla_{g} \psi\right\|_{g}^{2}+\left(\frac{1}{4} S c_{\alpha, \beta}+\frac{\beta}{4}\left\|\nabla_{g} f\right\|_{g}^{2}\right)\|\psi\|_{g}^{2}+\frac{\alpha}{4}\left\langle\nabla_{g} f, \nabla_{g}\|\psi\|_{g}^{2}\right\rangle_{g}\right] d \mathrm{Vol}_{g} .
\end{array}
$$

Then one gets

$$
\begin{aligned}
\frac{|\alpha|}{4}\left|\left\langle\nabla_{g} f, \nabla_{g}\|\psi\|_{g}^{2}\right\rangle_{g}\right| & \leq \frac{|\alpha|}{4}\left(c\left\|\nabla_{g} f\right\|_{g}\|\psi\|_{g} \times 2 c^{-1}\left\|\nabla_{g} \psi\right\|_{g}\right) \\
& \leq \frac{|\alpha| c^{2}}{8}\left\|\nabla_{g} f\right\|_{g}^{2}\|\psi\|_{g}^{2}+\frac{c^{-2}|\alpha|}{2}\left\|\nabla_{g} \psi\right\|_{g}^{2}
\end{aligned}
$$

Therefore,

$$
0 \geq \int_{M}\left[\left(1-\frac{c^{-2}|\alpha|}{2}\right)\left\|\nabla_{g} \psi\right\|_{g}^{2}+\frac{2 \beta-c^{2}|\alpha|}{8}\left\|\nabla_{g} f\right\|_{g}^{2}\|\psi\|_{g}^{2}+\frac{1}{4} S c_{\alpha, \beta}\|\psi\|_{g}^{2}\right] d \operatorname{Vol}_{g},
$$

where $c \neq 0$. If $c^{-2}|\alpha| \leq 2, \beta \geq \frac{c^{2}|\alpha|}{2}$ and $S c_{\alpha, \beta}>0$, then $\psi=0$. So the conditions $\alpha \in \mathbb{R}$ and $\beta \geq \frac{|\alpha|^{2}}{4}$ are needed.

$$
\begin{aligned}
\frac{|\alpha|}{4}\left|\left\langle\nabla_{g} f, \nabla_{g}\|\psi\|_{g}^{2}\right\rangle_{g}\right| & \leq \frac{|\alpha|}{4}\left(\left\|\nabla_{g} f\right\|_{g}\|\psi\|_{g} \times 2\left\|\nabla_{g} \psi\right\|_{g}\right) \\
& =\frac{|\alpha|}{2}\left(c_{1}\left\|\nabla_{g} f\right\|_{g}\|\psi\|_{g} \times c_{1}^{-1}\left\|\nabla_{g} \psi\right\|_{g}\right) \\
& \leq \frac{|\alpha|}{4}\left(c_{1}^{2}\left\|\nabla_{g} f\right\|_{g}^{2}\|\psi\|_{g}^{2}+c_{1}^{-2}\left\|\nabla_{g} \psi\right\|_{g}^{2}\right)
\end{aligned}
$$

Thus,

$$
0 \geq \int_{M}\left[\left(1-\frac{c_{1}^{-2}|\alpha|}{4}\right)\left\|\nabla_{g} \psi\right\|_{g}^{2}+\frac{\beta-c_{1}^{2}|\alpha|}{4}\left\|\nabla_{g} f\right\|_{g}^{2}\|\psi\|_{g}^{2}+\frac{1}{4} S c_{\alpha, \beta}\|\psi\|_{g}^{2}\right] d \mathrm{Vol}_{g},
$$

where $c_{1} \neq 0$. If $c_{1}^{-2}|\alpha| \leq 4, \beta \geq c_{1}^{2}|\alpha|$ and $S c_{\alpha, \beta}>0$, then $\psi=0$. Also the conditions $\alpha \in \mathbb{R}$ and $\beta \geq \frac{|\alpha|^{2}}{4}$ are needed. 
The following 3 corollaries come from the proposition of vanishing of harmonic spinors.

Corollary 4.4. Assume the smooth mm-space $\left(M^{n}, g, e^{-f} d \mathrm{Vol}_{g}\right)$ is closed and spin. If $\alpha \in \mathbb{R}, \beta \geq \frac{|\alpha|^{2}}{4}$ and $S c_{\alpha, \beta}>0$, then the $\widehat{A}$-genus and the Rosenberg index of $M^{n}$ vanish.

Proof. Since the $C^{*}\left(\pi_{1}\left(M^{n}\right)\right)$-bundle in the construction of the Rosenberg index [Ros83] is flat, there are no correction terms due to curvature of the bundle. Then the SchroedingerLichnerowicz-Weitzenboeck formula and the argument in the proof of vanishing of $\widehat{A}$-genus can be applied without change.

Corollary 4.5. Assume that $M^{n}$ is a closed spin n-manifold and $f$ is a smooth function on $M^{n}$. If one of the following conditions is met,

(1). $N \subset M^{n}$ is a codimension one closed connected submanifold with trivial normal bundle, the inclusion of fundamental groups $\pi_{1}\left(N^{n-1}\right) \rightarrow \pi_{1}\left(M^{n}\right)$ is injective and the Rosenberg index of $N$ does not vanish, or

(2). $N \subset M^{n}$ is a codimension two closed connected submanifold with trivial normal bundle, $\pi_{2}\left(M^{n}\right)=0$, the inclusion of fundamental groups $\pi_{1}\left(N^{n-1}\right) \rightarrow \pi_{1}\left(M^{n}\right)$ is injective and the Rosenberg index of $N$ does not vanish, or

(3). $N=N_{1} \cap \cdots \cap N_{k}$, where $N_{1} \cdots N_{k} \subset M$ are closed submanifolds that intersect mutually transversely and have trivial normal bundles. Suppose that the codimension $o N_{i}$ is at most two for all $i \in\{1 \ldots k\}$ and $\pi_{2}(N) \rightarrow \pi_{2}(M)$ is surjective and $\hat{A}(N) \neq 0$, then $M^{n}$ does not admit a Riemnannian metric $g$ such that $S c_{\alpha, \beta}>0$ for the dimension $n \geq 3, \alpha \in \mathbb{R}$ and $\beta \geq \frac{|\alpha|^{2}}{4}$ for the smooth mm-space $\left(M^{n}, g, e^{-f} d\right.$ vol $\left._{g}\right)$.

Proof. The results in the [HPS15, Theorem 1.1] and [Zei17, Theorem 1.9] can be applied to show that the Rosenberg index of $M^{n}$ does not vanish and Corollary 3.1 implies the theorem. 
Let $\mathcal{R}_{f}\left(M^{n}\right):=\{(g, f)\}$ be the space of densities, where $g$ is a smooth Riemannian metric on $M^{n}$ and $f$ is a smooth function on $M^{n}$ and $\mathcal{R}_{f}^{+}\left(M^{n}\right) \subset \mathcal{R}_{f}\left(M^{n}\right)$ is the subspace of densities such that the smooth mm-space $\left(M^{n}, g, e^{-f} d v o l_{g}\right)$ satisfies $S c_{\alpha, \beta}>0$. Furthermore, let $\mathcal{R}_{f}^{+}\left(M^{n}\right)$ be endowed with the smooth topology.

Corollary 4.6. Assume $M^{n}$ is a closed spin n-manifold, $n \geq 3, \alpha \in \mathbb{R}$ and $\beta \geq \frac{|\alpha|^{2}}{4}$ and $\mathcal{R}_{f}^{+}\left(M^{n}\right) \neq \emptyset$, then there exist a homomorphism

$$
A_{m-1}: \pi_{m-1}\left(\mathcal{R}_{f}^{+}\left(M^{n}\right)\right) \rightarrow K O_{n+m}
$$

such that

- $A_{0} \neq 0$, if $n \equiv 0,1(\bmod 8)$

- $A_{1} \neq 0$, if $n \equiv-1,0(\bmod 8)$

- $A_{8 j+1-n} \neq 0$, if $n \geq 7$ and $8 j-n \geq 0$.

Proof. Since the results in the [Hit74, Section 4.4] and [CS13] depend on the existing of exotic spheres with non-vanishing $\alpha$-invariant. Let $\phi: M^{n} \rightarrow M^{n}$ be a diffeomorphism of $M^{n}$ and $\left.(g, f) \in \mathcal{R}_{f}^{+}\left(M^{n}\right)\right)$, then $\left(\phi^{*} g, f \circ \phi\right)$ is also in $\mathcal{R}_{f}^{+}\left(M^{n}\right)$. Combining it with Proposition 4.3 show that Hitch's construction of the map $A$ [Hit74, Proposition 4.6] can be applied to the case of $\mathcal{R}_{f}^{+}\left(M^{n}\right)$ and then we can finish the proof with the arguments in [Hit74, Section 4.4] and [CS13, Section 2.5].

\subsection{Conformal to PSC-metrics}

The scalar curvature is well behave under the conformal change of a Riemannian metric such that one can use it as a tool to attend problems about the scalar curvature. The Yamabe problem and the Yamabe invariant [LP87] are the well-know examples. We also consider the change of the weighted scalar curvature under a conformal change of a Riemannian metric. 
Proposition 4.7 (Conformal to PSC-metrics). Let $\left(M^{n}, g, e^{-f} d \mathrm{Vol}_{g}\right)$ be a closed smooth mm-space with $S c_{\alpha, \beta}>0$. If the dimension $n \geq 3, \alpha \in \mathbb{R}$ and $\beta \geq \frac{(n-2)|\alpha|^{2}}{4(n-1)}$, then there is $a$ metric $\tilde{g}$ conformal to $g$ with positive scalar curvature (PSC-metric).

Proof. One only needs to show for all nontrivial $u, \int_{M}-u L_{g} u d \operatorname{Vol}_{g}>0$ as in the Yamabe problem [Sch84], where

$$
L_{g}:=\triangle_{g}-\frac{n-2}{4(n-1)} S c_{g}
$$

is conformal Laplacian operator. To see this,

$$
\begin{array}{r}
\int_{M}-u L_{g} u d \mathrm{Vol}_{g}=\int_{M}\left[\left\|\nabla_{g} u\right\|_{g}^{2}+\frac{n-2}{4(n-1)} S c_{g} u^{2}\right] d \mathrm{Vol}_{g} \\
=\int_{M}\left[\left\|\nabla_{g} u\right\|_{g}^{2}+\frac{n-2}{4(n-1)}\left(S c_{\alpha, \beta}-\alpha \Delta_{g} f+\beta\left\|\nabla_{g} f\right\|_{g}^{2}\right) u^{2}\right] d \mathrm{Vol}_{g} \\
=\int_{M}\left[\left\|\nabla_{g} u\right\|_{g}^{2}+\frac{n-2}{4(n-1)}\left(S c_{\alpha, \beta}+\beta\left\|\nabla_{g} f\right\|_{g}^{2}\right) u^{2}+\frac{\alpha(n-2)}{2(n-1)}\left\langle\nabla_{g} f, \nabla_{g} u\right\rangle_{g} u\right] d \mathrm{Vol}_{g}
\end{array}
$$

Through the inequality

$$
\left\langle\nabla_{g} f, \nabla_{g} u\right\rangle_{g} u \leq c_{2}\left\|\nabla_{g} f\right\|_{g} u \times c_{2}^{-1}\left\|\nabla_{g} u\right\|_{g} \leq \frac{c_{2}^{2}\left\|\nabla_{g} f\right\|_{g}^{2} u^{2}+c_{2}^{-2}\left\|\nabla_{g} u\right\|_{g}^{2}}{2},
$$

one gets

$$
\begin{aligned}
\int_{M}-u L_{g} u d \operatorname{Vol}_{g} \geq & \\
& \int_{M}\left[\left(1-\frac{|\alpha| c_{2}^{-2}(n-2)}{4(n-1)}\right)\left\|\nabla_{g} u\right\|_{g}^{2}+\right. \\
& \left.\frac{\left(\beta-|\alpha| c_{2}^{-2}\right)(n-2)}{4(n-1)}\left\|\nabla_{g} f\right\|_{g}^{2} u^{2}+\frac{n-2}{4(n-1)} S c_{\alpha, \beta} u^{2}\right] d \operatorname{Vol}_{g}
\end{aligned}
$$

where $c_{2} \neq 0$.

If $|\alpha| c_{2}^{-2} \leq \frac{4(n-1)}{n-2}, \beta \geq c_{2}^{2}|\alpha|$ and $S c_{\alpha, \beta}>0$, then

$$
\int_{M}-u L_{g} u d \operatorname{Vol}_{g}>0
$$

So the conditions $n>2, \alpha \in \mathbb{R}$ and $\beta \geq \frac{(n-2) \alpha^{2}}{4(n-1)}$ are needed. 
Remark 4.8. The proof was borrowed from [AC17, Proposition 2]. The two propositions above offer a geometric reason why the condition of the vanishing of $\widehat{A}$-genus (without simply connected condition) does not imply that $M^{n}$ can admit a PSC-metric for the closed spin manifold $M^{n}$.

The proposition of conformal to PSC-metrics has following 3 corollaries.

Corollary 4.9 (Weighted spherical Lipschitz bounded).

Let $\left(M^{n}, g, e^{-f} d \mathrm{Vol}_{g}\right)$ be a closed orientable smooth mm-space with $S c_{\alpha, \beta} \geq \kappa>0$, $3 \leq n \leq 8, \alpha \in \mathbb{R}$ and $\beta \geq \frac{(n-2)|\alpha|^{2}}{4(n-1)}$, then the Lipschitz constant of the continuous map $\phi$ from $\left(M^{n}, g, e^{-f} d \mathrm{Vol}_{g}\right)$ to the sphere $S^{n}$ with standard metric of non-zero degrees has uniformly non-zero lower bounded.

Proof. There is a metric $\tilde{g}$ conformal to $g$ with scalar curvature $\geq n(n-1)$ by the proposition of conformal PSC-metrics. For the continuous map $\phi$ from $\left(M^{n}, \tilde{g}\right)$ to $S^{n}$ of non-zero degrees, the Lipschitz constant of $\phi$ is greater than a constant that depends only on the dimensions $n$ by Gromov's spherical Lipschitz bounded theorem [Gro18, Section 3]. Since the conformal function has the positive upper bound by the compactness of the manifold, then the Lipschitz constant has uniformly non-zero lower bounded.

Corollary 4.10. For closed smooth mm-spaces $\left(M^{n}, g, e^{-f} d \mathrm{Vol}_{g}\right)(n \geq 3)$ satisfying with $S c^{v^{o l} l_{n}}\left(M^{n}\right)>0$, there is a metric $\hat{g}$ conformal to $g$ with PSC-metric. In particular, the $\widehat{A}$-genus and Rosenberg index vanish with additional spin condition.

For closed orientable smooth mm-spaces $\left(M^{n}, g, e^{-f} d \operatorname{Vol}_{g}\right)(3 \leq n \leq 8)$ satisfying with $S c^{\text {vol }_{n}}\left(M^{n}\right) \geq \kappa>0$, then the Lipschitz constant of the continuous map $\phi$ from $\left(M^{n}, g, e^{-f} d \mathrm{Vol}_{g}\right)$ to the sphere $S^{n}$ with standard metric of non-zero degrees has uniformly non-zero lower bounded.

Proof. The volume of the small disk of $\left(M^{n}, g, e^{-f} d \mathrm{Vol}_{g}\right)$ was computed in [Qia97, Theo- 
rem 8],

$$
\mu\left(B_{r}(x)\right)=\operatorname{vol}_{E}\left(B_{r}\left(\mathbb{R}^{n}\right)\right)\left[1-\frac{S c_{g}+3 \triangle_{g} f-3\left\|\nabla_{g} f\right\|_{g}^{2}}{6(n+2)} r^{2}+O\left(r^{4}\right)\right]
$$

as $r \rightarrow 0$. Since $S c^{v o l_{n}}\left(M^{n}\right)>0$, i.e. $\mu\left(B_{r}(x)\right)<\operatorname{vol}_{E}\left(B_{r}\right)$ as $r \rightarrow 0$, then

$$
S c_{g}+3 \triangle_{g} f-3\left\|\nabla_{g} f\right\|_{g}^{2}>0 \text {. }
$$

Therefore, the propositions of vanishing harmonic spinors and of conformal PSC-metrics and Corollary 4.9 imply it.

Remark 4.11. Since any weighted Riemannian manifold (with non-trivial Borel measure) is infinitesimally Hilbertian (See, [LP20]), Corollary 4.10 also works for $\left(M^{n}, g, e^{-f} d \mathrm{Vol}_{g}\right)$ with $R S C(\kappa, n)$ condition.

Enlargeability as an obstruction to the existence of a PSC-metric on a closed manifold was introduced by Gromov-Lawson. We call a manifold enlargeable as Gromov-Lawson's definition in [GL83, Definition 5.5]

Corollary 4.12. Assume $M^{n}(n \geq 3)$ is a closed spin smooth enlargeable manifold, then $\mathcal{R}_{f}^{+}\left(M^{n}\right)$ is an empty set for $\alpha \in \mathbb{R}$ and $\beta \geq \frac{(n-2)|\alpha|^{2}}{4(n-1)}$.

In particular, ( $\left.\mathbb{T}^{n}, g, e^{-f} d \mathrm{Vol}_{g}\right)$ does not satisfy $S c^{\text {vol }_{n}}\left(\mathbb{T}^{n}\right)>0$ for any $C^{2}$-smooth Riemannian metrics $g$ and $C^{2}$-smooth functions $f$ on the torus $\mathbb{T}^{n}$.

Proof. Since a closed enlargeable manifold cannot carry a PSC-metric [GL83, Theorem 5.8], Proposition 4.7 implies $\mathcal{R}_{f}^{+}\left(M^{n}\right)=\emptyset$ for $\alpha \in \mathbb{R}$ and $\beta \geq \frac{(n-2)|\alpha|^{2}}{4(n-1)}$.

$\mathbb{T}^{n}$ is an important example of enlargeable manifolds and then Corollary 4.10 implies that $\left(\mathbb{T}^{n}, g, e^{-f} d \operatorname{Vol}_{g}\right)$ does not satisfy $S c^{v^{v o l} n}\left(\mathbb{T}^{n}\right)>0$ for $n \geq 3$. For dimension 2 , the conditions of $S c_{\alpha, \beta}>0$ and $\beta \geq 0$ imply that the oriented surface is 2 -sphere. 


\section{3 $f$-minimal hypersurface and $S c_{\alpha, \beta}>0$}

In addition to using the Dirac operator method, Schoen-Yau's minimal hypersurface method $[\mathrm{SY79b}]$ is another main idea. For an immersed orientable hypersurface $N^{n-1} \subset M^{n}$, the weighted mean curvature vector $H_{f}$ of $N^{n-1}$ is defined by Gromov in [Gro03, 9.4 E],

$$
H_{f}=H+\left(\nabla_{g} f\right)^{\perp}
$$

where $H$ is the mean curvature vector field of the immersion, $(\cdot)^{\perp}$ is the projection on the normal bundle of $N^{n-1}$. The first and second variational formulae for the weighted volume functional of $N^{n-1}$ were derived in Bayle's thesis (also see [Ros+08]). We take the detailed presentation of such derivation for [CMZ15]. The $\left(N^{n-1}, \bar{g}\right)$ with the induced metric is called $f$-minimal hypersurface if the weighted mean curvature vector $H_{f}$ vanishes identically.

In fact, the definition of $f$-minimal hypersurface can also be derived from the first variational formula. Furthermore, an $f$-minimal hypersurface is a minimal hypersurface of $\left(M^{n}, \tilde{g}\right)$, where $\tilde{g}$ is the conformal metric of $g, \tilde{g}=e^{-\frac{2 f}{n-1}} g$.

The connection between the geometry of the ambient smooth mm-space and the $f$ minimal hypersurfaces occurs via the second variation of the weighted volume functional. For a hypersurface $\left(N^{n-1}, \bar{g}\right)$, the $L_{f}$ operator is defined by

$$
L_{f}:=\triangle_{f}+|A|^{2}+\operatorname{Ricc}_{f}^{M}(v, v)
$$

where $v$ is the unit normal vector, $|A|^{2}$ denotes the square of the norm of the second fundamental form $A$ of $N^{n-1}$ and

$$
\triangle_{f}:=\triangle_{\bar{g}}-\left\langle\nabla_{\bar{g}} f, \nabla_{\bar{g}}\right\rangle
$$

is the weighted Laplacian. Through the second variational formula, a two-sided $f$-minimal hypersurface $N^{n-1}$ is stable (called $L_{f}$-stable) if for any compactly supported smooth 
function $u \in C_{c}^{\infty}\left(N^{n-1}\right)$, it holds that

$$
-\int_{N} u L_{f} u e^{-f} d \operatorname{Vol}_{\bar{g}} \geq 0
$$

Proposition 4.13. Let $\left(M^{n}, g, e^{-f} d \mathrm{Vol}_{g}\right)$ be a closed orientable smooth mm-space with $S c_{\alpha, \beta}>0$ and $\left(N^{n-1}, \bar{g}\right)$ be the compact $L_{f}$-stable minimal hypersurface of $\left(M^{n}, g, e^{-f} d \mathrm{Vol}_{g}\right)$. If the dimension $n \geq 3, \alpha=2$, and $\beta \geq \frac{n-2}{n-1}$, then there exists a PSC-metric conformal to $\bar{g}$ on $N^{n-1}$.

Proof. The $f$-minimal hypersurface $\left(N^{n-1}, \bar{g}\right)$ is $L_{f}$-stable if and only if $\left(N^{n-1}, \overline{\tilde{g}}\right)$ is stable as a minimal hypersurface on $\left(M^{n}, \tilde{g}\right)$, where $\tilde{g}:=e^{-\frac{2 f}{n-1}} g$ and $\overline{\tilde{g}}$ is the induced metric of $\tilde{g}$ (See [CMZ15, Appendix]). On the other hand, the scalar curvature of $\left(M^{n}, \tilde{g}\right)$ is

$$
S c_{\tilde{g}}=e^{\frac{f}{n-1}}\left(S c_{g}+2 \triangle_{g} f-\frac{n-2}{n-1}\left\|\nabla_{g} f\right\|_{g}^{2}\right)
$$

Thus, $S c_{\alpha, \beta}>0$ with $n \geq 3, \alpha=2$, and $\beta \geq \frac{n-2}{n-1}$ imply $S c_{\tilde{g}}>0$. Then the standard Schoen-Yau's argument can be applied to show that $\overline{\tilde{g}}$ conformal to a PSC-metric on $N^{n-1}$.

Remark 4.14. The minimal hypersurface method poses a stricter condition to the valid range of $\alpha, \beta$ than that of the Dirac operator method.

Since the oriented closed manifolds with a PSC-metric in 2 and 3 dimensions are classified by Gauess-Bonnet theorem and Perelman-Thurston geometrization theorem, then Proposition 3.3 can give the following elementary applications:

Corollary 4.15. Let $\left(M^{n}, g, e^{-f} d \mathrm{Vol}_{g}\right)$ be a closed orientable smooth mm-space with $S c_{\alpha, \beta}>0$.

(i) If $n=3, \alpha=2$, and $\beta \geq \frac{1}{2}$, then there is no closed immersed $L_{f}$-stable minimal 2 dimensional surface with positive genus. 
(ii) If $n=4, \alpha=2$, and $\beta \geq \frac{2}{3}$, then the closed immersed $L_{f}$-stable minimal 3 dimensional submanifold must be spherical 3-manifolds, $S^{2} \times S^{1}$ or the connected sum of spherical 3-manifolds and copies of $S^{2} \times S^{1}$.

The prototype of Corollary 4.15(1) is the Schoen-Yau's classic result, which said that the oriented closed 3-manifold with a PSC-metric has no compact immersed stable minimal surface of positive genus [SY79b]. The Schoen-Yau result had been generalized to Perelman's P-scalar curvature $>0$ by Fan [Fan08]. Note that one can also consider the noncompact immersed $L_{f}$-stable minimal 2 dimensional surface under the condition of Corollary 4.15 (1) since an oriented complete stable minimal surface in a complete oriented 3-manifold with a PSC-metric is conformally equivalent to the complex plane $\mathbb{C}$ showed by Fischer-Colbrie-Schoen [FCS80].

The smooth mm-space with $S c_{\alpha, \beta}>0$ under suit ranges of $\alpha$ and $\beta$ implies the manifold admits PSC-metrics, but the manifold (itself) that can admit PSC-metrics does not necessarily imply there exists $S c_{\alpha, \beta}>0$.

Question 4.16. Does the smooth mm-space with $S c_{\alpha, \beta}>0$ under suitable ranges of $\alpha$ and $\beta$ give more topological restriction on the manifold than the PSC-metric on the manifold?

Question 4.17. Let $M$ be a closed smooth manifold, $f$ be a smooth function on $M$ and $h$ be a smooth function that is negative for some point $p$ on $M$. What is the range of $\alpha$ and $\beta$ such that there exists a smooth Riemannian metric $g$ on $M$ satisfying

$$
S c_{g}+\alpha \triangle_{g} f-\beta\left\|\nabla_{g} f\right\|_{g}^{2}=h
$$

i.e. $S c_{\alpha, \beta}(g)=h$ ?

Let $\left(M, g_{i}\right)$ be smooth Riemannian manifolds and $\left\{g_{i}\right\}_{i \in \mathbb{N}} C^{0}$-converges to $g$, then $\left\{g_{i}\right\}_{i \in \mathbb{N}}$ also smGH-converges to $g$. Gromov showed that the scalar curvature $\geq \kappa$ is stable under $C^{0}$-convergence in [Gro14, Section 1.8]. 
Question 4.18. Assume smooth mm-spaces $\left(M^{n}, g_{i}, e^{-f} d \operatorname{Vol}_{g_{i}}\right)$ all satisfy $S c^{\text {vol }_{n}}\left(M^{n}\right) \geq 0$ such that $\left\{g_{i}\right\}_{i \in \mathbb{N}} C^{2}$-converges to the smooth Riemannian metric $g$ on $M^{n}$, then does $\left(M^{n}, g, e^{-f} d \mathrm{Vol}_{g}\right)$ also satisfy $\operatorname{Sc}^{\text {vol }_{n}}\left(M^{n}\right) \geq 0$ ?

Question 4.19. Let mm-spaces $\left(M^{n}, g, e^{-f} d \operatorname{Vol}_{g}\right)$ with $S c^{\text {vol }}\left(M^{n}\right) \geq \kappa>0$, where $M^{n}$ are closed smooth manifold, $g$ and $f$ are $C^{0}$-smooth, then does there exist a PSC-metric on $M^{n}$ ?

Since the role of $S c_{\alpha, \beta}>0$ on the smooth mm-space is similar to the role of $S c>0$ on the manifold, one can try to extend the knowledge about $S c>0$ to $S c_{\alpha, \beta}>0$.

\subsection{Weighted rigidity}

Gromov's conjecture that said if a smooth Riemannian metric $g$ satisfies $g \geq g_{s t}$ and $S c(g) \geq S c\left(g_{s t}\right)=n(n-1)$ on $S^{n}$ then $g=g_{s t}$, was proved by Llarull [Lla98] and called Llarull rigidity theorem. A map $h:\left(M^{n}, g_{M}\right) \rightarrow\left(N^{n}, g_{N}\right)$ is said to be $\epsilon$-contracting if $\left\|h_{*} v\right\|_{g_{N}} \leq \epsilon\|v\|_{g_{N}}$ for all tangent vectors $v$ on $M^{n}$

Proposition 4.20 (Weighted rigidity). Assume the smooth mm-space $\left(M^{n}, g, e^{-f} d \mathrm{Vol}_{g}\right)$ is closed and spin and there exists a smooth 1-contracting map $h:\left(M^{n}, g\right) \rightarrow\left(S^{n}, g_{s t}\right)$ of non-zero degree. If $\alpha \in \mathbb{R}, \beta \geq \frac{|\alpha|^{2}}{4}$ and $S c_{\alpha, \beta} \geq n(n-1)$, then $h$ is an isometry between the metrics $g$ and $g_{s t}$. Furthermore, if $\alpha>0$, then $f$ is a constant function.

Proof. One just need to insert the tricks in the proof of Proposition 4.3 to the proof in [Lla98, Theorem 4.1]. Following the setup of LLarull, we only prove the even dimensional $2 n$ case without loss of generality.

First, we will show that $h$ is an isometry. Fix $p \in M^{2 n}$. Let $\left\{e_{1}, \ldots, e_{2 n}\right\}$ be a $g$ orthonormal tangent fame near $p$ such that $\left(\nabla_{g} e_{k}\right)_{p}=0$ for each $k$. Let $\left\{\epsilon_{1}, \ldots, \epsilon_{2 n}\right\}$ be a $g_{s t}$-orthonormal tangent frame near $f(p) \in S^{2 n}$ such that $\left(\nabla_{g_{s t}} \epsilon_{k}\right)_{h(p)}=0$ for each $k$. 
Moreover, the bases $\left\{e_{1}, \ldots, e_{2 n}\right\}$ and $\left\{\epsilon_{1}, \ldots, \epsilon_{2 n}\right\}$ can be chosen so that $\epsilon_{j}=\lambda_{j} h_{*} e_{j}$ for appropriate $\left\{\lambda_{j}\right\}_{j=1}^{2 n}$. This is possible since $h_{*}$ is symmetric. Since $h$ is 1-contracting map, $\lambda_{k} \geq 1$ for each $k$.

Then one constructs the twisted vector bundles $S \otimes E$ over $M^{2 n}$ as Llarull did. Let $R^{E}$ be the curvature tensor of $E$ and $\psi$ be a twisted spinor, then one gets

$$
\left\langle R^{E} \psi, \psi\right\rangle_{g} \geq-\frac{1}{4} \sum_{i \neq j} \frac{1}{\lambda_{i} \lambda_{j}}\|\psi\|_{g} .
$$

For the twisted Dirac operator $\mathbb{D}_{E}$, one has $\mathbb{D}_{E}^{2}=\nabla^{*} \nabla+\frac{1}{4} S c_{g}+R^{E}$ and

$$
\begin{array}{r}
\int_{M}\left\langle\mathbb{D}_{E}^{2} \psi, \psi\right\rangle_{g} d \operatorname{Vol}_{g}=\int_{M}\left[\left\|\nabla_{g} \psi\right\|_{g}^{2}+\frac{1}{4}\left(S c_{\alpha, \beta}-\alpha \Delta_{g} f+\beta\left\|\nabla_{g} f\right\|_{g}^{2}\right)\|\psi\|_{g}^{2}+\left\langle R^{E} \psi, \psi\right\rangle_{g}\right] d \mathrm{Vol}_{g} \\
=\int_{M}\left[\left\|\nabla_{g} \psi\right\|_{g}^{2}+\left(\frac{1}{4} S c_{\alpha, \beta}+\frac{\beta}{4}\left\|\nabla_{g} f\right\|_{g}^{2}\right)\|\psi\|_{g}^{2}+\frac{\alpha}{4}\left\langle\nabla_{g} f, \nabla_{g}\|\psi\|_{g}^{2}\right\rangle_{g}+\left\langle R^{E} \psi, \psi\right\rangle_{g}\right] d \mathrm{Vol}_{g} .
\end{array}
$$

Because $\lambda_{k} \geq 1$ for each $k$, one gets

$$
\left\langle R^{E} \psi, \psi\right\rangle_{g} \geq \frac{-2 n(2 n-1)}{4}\|\psi\|_{g}
$$

and then

$$
\begin{aligned}
\frac{|\alpha|}{4}\left|\left\langle\nabla_{g} f, \nabla_{g}\|\psi\|_{g}^{2}\right\rangle_{g}\right| & \leq \frac{|\alpha|}{4}\left(\left\|\nabla_{g} f\right\|_{g}\|\psi\|_{g} \times 2\left\|\nabla_{g} \psi\right\|_{g}\right) \\
& =\frac{|\alpha|}{2}\left(c_{1}\left\|\nabla_{g} f\right\|_{g}\|\psi\|_{g} \times c_{1}^{-1}\left\|\nabla_{g} \psi\right\|_{g}\right) \\
& \leq \frac{|\alpha|}{4}\left(c_{1}^{2}\left\|\nabla_{g} f\right\|_{g}^{2}\|\psi\|_{g}^{2}+c_{1}^{-2}\left\|\nabla_{g} \psi\right\|_{g}^{2}\right)
\end{aligned}
$$

where $c_{1} \neq 0$. Therefore,

$$
\begin{aligned}
& \int_{M}\left\langle\mathbb{D}_{E}^{2} \psi, \psi\right\rangle_{g} d \operatorname{Vol}_{g} \geq \\
& \int_{M}\left[\left(1-\frac{c_{1}^{-2}|\alpha|}{4}\right)\left\|\nabla_{g} \psi\right\|_{g}^{2}+\frac{\beta-c_{1}^{2}|\alpha|}{4}\left\|\nabla_{g} f\right\|_{g}^{2}\|\psi\|_{g}^{2}+\right. \\
&\left.\frac{1}{4}\left(S c_{\alpha, \beta}-2 n(2 n-1)\right)\|\psi\|_{g}^{2}\right] d \mathrm{Vol}_{g} .
\end{aligned}
$$


Furthermore, since $\alpha \in \mathbb{R}, \beta \geq \frac{|\alpha|^{2}}{4}$ and $S c_{\alpha, \beta} \geq 2 n(2 n-1)$, one can choose $c_{1}$ such that $c_{1}^{-2}|\alpha| \leq 4$, then $\beta-c_{1}^{2}|\alpha| \geq 0$. Thus,

$$
\int_{M}\left\langle\mathbb{D}_{E}^{2} \psi, \psi\right\rangle_{g} d \operatorname{Vol}_{g} \geq \int_{M} \frac{1}{4}\left[S c_{\alpha, \beta}-2 n(2 n-1)\right]\|\psi\|_{g}^{2} d \operatorname{Vol}_{g} \geq 0
$$

The fact $\operatorname{Index}\left(\mathbb{D}_{E^{+}}\right) \neq 0$ implies $\operatorname{ker}\left(\mathbb{D}_{E}\right) \neq 0$ and then $S c_{\alpha, \beta}=2 n(2 n-1)$. Then using the inequality $\left\langle R^{E} \psi, \psi\right\rangle_{g} \geq-\frac{1}{4} \sum_{i \neq j} \frac{1}{\lambda_{i} \lambda_{j}}\|\psi\|_{g}$, one gets

$$
\int_{M}\left\langle\mathbb{D}_{E}^{2} \psi, \psi\right\rangle_{g} d \operatorname{Vol}_{g} \geq \int_{M} \frac{1}{4}\left[\sum_{i \neq j}\left(1-\frac{1}{\lambda_{i} \lambda_{j}}\right)\right]\|\psi\|_{g}^{2} d \operatorname{Vol}_{g} \geq 0 .
$$

Choosing $\psi \neq 0$ such that $D_{E} \psi=0$, one has

$$
0 \leq 1-\frac{1}{\lambda_{i} \lambda_{j}} \leq 0
$$

for $i \neq j$. Thus, $\lambda_{k}=0$ for all $1 \leq k \leq 2 n$ and $h$ is an isometry.

Second, we will show that $f$ is a constant function. Since $S c_{\alpha, \beta}=2 n(2 n-1), \alpha>0$, $\beta \geq \frac{|\alpha|^{2}}{4}$ and $S c_{g}=2 n(2 n-1)$, then $\triangle_{g} f \geq 0$. One has

$$
\int_{M} \triangle_{g} f d \mathrm{Vol}_{g}=0
$$

for a closed manifold $M^{n}$, so one gets $\Delta_{g} f=0$. That implies $\nabla_{g} f=0$ so that $f$ is a constant function on $M^{n}$.

Corollary 4.21. Let closed and spin smooth mm-spaces $\left(M^{n}, g, e^{-f} d \mathrm{Vol}_{g}\right)$ satisfying with $S c^{\text {vol }_{n}}\left(M^{n}\right) \geq n(n-1)$ and there exists a smooth 1-contracting map $h:\left(M^{n}, g\right) \rightarrow\left(S^{n}, g_{s t}\right)$ of non-zero degree, then $h$ is an isometry between the metrics $g$ and $g_{s t}$.

Proof. Combining the weighted rigidity theorem and the proof of Corollary 4.10 can imply it.

As Llarull rigidity theorem (and the weighted rigidity theorem) still holds if the condition that $h$ is 1 -contracting is replaced by the condition that $h$ is area-contracting, Gromov 60 
called such metrics area-extremal metrics, asked which manifolds possess area-extremal metrics, and conjectured that Riemannian symmetric spaces should have area-extremal metrics [Gro96], [Gro17, Section 17] and [Gro19, Section 4.2]. Goette-Semmelmann showed that several classes of symmetric spaces with non-constant curvatures are area-extremal [GS02].

Question 4.22. Can Goette-Semmelmann's results [GS02] be generalized to the smooth mm-space with $S c_{\alpha, \beta}>0$ under other suitable conditions?

Since Corollary 4.15 (1) showed that the closed orientable immersed $L_{f}$-stable minimal 2 dimensional surface in the closed orientable smooth mm-space $\left(M^{n}, g, e^{-f} d \mathrm{Vol}_{g}\right)$ with $S c_{2, \beta}>0\left(\beta \geq \frac{1}{2}\right)$ is 2 -sphere, then one can consider rigidity of area-minimizing 2-sphere in 3 dimensional smooth mm-space. Bray's volume comparison theorem [Bra97, Chapter 3, Theorem 18] is another rigidity theorem that needs the conditions of Ricci curvature and scalar curvature bounded below. There are other rigidity phenomena involving scalar curvature, see [Bre12].

Question 4.23. Can Bray's volume comparison theorem be extended to the smooth mmspace? 



\section{Weighted Scalar Curvature in Four Dimensions}

In this chapter, our goal is to study the weighted scalar curvature in four dimensions. We single out 4 dimensions because it is very special dimension in topology and physics. In particular, Seiberg-Witten invariants only exist in dimensional 4 and we focus on SeibergWitten invariants and General relativity. We will show how the weighted scalar curvature relates to them.

\subsection{Seiberg-Witten invariants and weighted scalar curva- ture}

Besides Schoen-Yau'S and Gromov-Lawson's fundamental approaches, using SeibergWitten invariants (SW-invariants) as an obstruction of existence of positive scalar curvature is another method in four dimensions.

The SW-invariants are defined a priori for a compact, oriented 4-manifold $M$ with the characteristic number $b_{2}^{+}>1$. There is a more complicated structure in the case where $b_{2}^{+}=1$. Here, $b_{2}^{+}$is equal to the number of +1 eigenvalues of the intersection form on the (rational) second homology. Thus, $b_{2}^{+}=\frac{1}{2}\left(\operatorname{rank}\left(H_{2}(M)+\operatorname{sign}(M)\right)\right.$, where $\operatorname{sign}(M)$ is the signature of $M$. 
The SW-invariants of $M$ constitute a map from the set of equivalence classes of $\operatorname{spin}^{c}$ structures on $M$ (covering the frame bundle) to the integers. These invarants are defined by counting (in a suitable sense) the solutions of a natural system of differential equations on $M$ which are defined by using a $\operatorname{spin}^{c}$ structure.

Theorem 5.1 (Witten [Wit94]). Assume that a closed oriented smooth 4-manifold M admits a Riemannian metric with positive scalar curvature, then the Seiberg-Witten invariants of M are zero.

Furthermore, there exists a closed simply connected non-spin 4-manifold with nonvanishing $\mathrm{SW}$-invariants. For example, the manifold

$$
X(2 k+1):=\left\{\left[z_{0}: z_{1}: z_{2}: z_{3}\right] \in \mathbb{C} P^{3} \mid z_{0}^{2 k+1}+z_{1}^{2 k+1}+z_{2}^{2 k+1}+z_{3}^{2 k+1}=0, k \geq 2\right\}
$$

is a simply connected non-spin manifold of real dimension 4. Thus, the index theory method and the minimal hypersurface method do not apply. However, its SW-invariants are non-zero by Taubes' result [Tau94] and then it does not support a PSC-metric.

For the weighted Riemannian 4-manifold $\left(M, g, e^{-f} d \operatorname{vol}_{g}\right)$, where $M$ is a closed oriented 4-manifold and $g$ is a smooth Riemannian metric and $f$ is a smooth function on $M$, we show that positive weighted scalar curvature $S c_{\alpha, \beta}>0$ with certain conditions on $\alpha$ and $\beta$ implies the vanishing of SW-invariants in the following theorem.

Theorem 5.2. Assume that the weighted Riemannian 4-manifold $\left(M, g, e^{-f} d \mathrm{vol}_{g}\right)$ with $\alpha \in \mathbb{R}, \beta \geq \frac{|\alpha|^{2}}{4}$, and $S c_{\alpha, \beta}>0$, then the Seiberg-Witten invariants of $M$ are zero.

Proof. Since a closed oriented 4-manifold admits $\operatorname{Spin}^{c}$ structures by an old result of Hirzebruch and Hopf [HH58], one can choose a $\operatorname{Spin}^{c}$ structure on $M$. Following the set up in [Don96], the $\operatorname{Spin}^{c}$ structure is given by a pair of vector bundles $W^{ \pm}$over $M$ with an isomorphism $\Lambda^{2} W^{+}=\Lambda^{2} W^{-}=L$. Thus, $W^{ \pm}$are $U(2)$-bundles over $M$ and a connection on $L$ gives a Dirac operator $D: \Gamma\left(W^{+}\right) \rightarrow \Gamma\left(W^{-}\right)$. Then there is a sequilinear 
map $\tau: \Gamma\left(W^{+}\right) \times \Gamma\left(W^{+}\right) \rightarrow \Lambda^{+}(M) \otimes \mathbb{C}$, where $\Lambda^{+}(M)$ is the self-dual 2-forms of $M$, such that one has $\tau(\psi, \psi)=\frac{1}{2}\|\psi\|_{g}^{2} \psi$ for a section $\psi$ of $W^{+}$.

Let $A$ be a unitary connection on $L$, and $F_{A}^{+}$be the self-dual part of the curvature of $A$, then the Seiberg-Witten equations for the pair $(A, \psi)$ are:

$$
\begin{array}{r}
D_{A} \psi=0, \\
F_{A}^{+}=-\tau(\psi, \psi) .
\end{array}
$$

One has the Schroedinger-Lichnerowicz-Weitzenboeck formula

$$
D_{A}^{*} D_{A} \psi=\nabla_{A}^{*} \nabla_{A} \psi+\frac{1}{4} S c_{g} \psi-\frac{1}{2} F_{A}^{+}(\psi)
$$

Suppose $(A, \psi)$ is a solution of the Seiberg-Witten equations over $M$ and then take the $L^{2}$ inner product with $\psi$, one gets

$$
0=\int_{M}\left[\left\|\nabla_{A} \psi\right\|_{g}^{2}+\frac{1}{4} S c_{g}\|\psi\|_{g}^{2}-\frac{1}{2}\left\langle F_{A}^{+}(\psi), \psi\right\rangle_{g}\right] d \mathrm{Vol}_{g}
$$

and

$$
\left\langle F_{A}^{+}(\psi), \psi\right\rangle_{g}=-\langle\tau(\psi, \psi)(\psi), \psi\rangle_{g}=-\frac{1}{2}\|\psi\|_{g}^{4}
$$

by the first and second equation of Seiberg-Witten and the property of $\tau$,

$$
\tau(\psi, \psi)=\frac{1}{2}\|\psi\|_{g}^{2} \psi
$$

Therefore, one has

$$
0=\int_{M}\left[\left\|\nabla_{A} \psi\right\|_{g}^{2}+\frac{1}{4} S c_{g}\|\psi\|_{g}^{2}+\frac{1}{4}\|\psi\|_{g}^{4}\right] d \operatorname{Vol}_{g}
$$

If $\alpha \in \mathbb{R}, \beta \geq \frac{|\alpha|^{2}}{4}$ and $S c_{\alpha, \beta}>0$,

$$
\begin{array}{r}
0=\int_{M}\left[\left\|\nabla_{A} \psi\right\|_{g}^{2}+\frac{1}{4}\|\psi\|_{g}^{4}+\frac{1}{4}\left(S c_{\alpha, \beta}-\alpha \Delta_{g} f+\beta\left\|\nabla_{g} f\right\|_{g}^{2}\right)\|\psi\|_{g}^{2}\right] d \operatorname{Vol}_{g} \\
=\int_{M}\left[\left\|\nabla_{A} \psi\right\|_{g}^{2}+\frac{1}{4}\|\psi\|_{g}^{4}+\left(\frac{1}{4} S c_{\alpha, \beta}+\frac{\beta}{4}\left\|\nabla_{g} f\right\|_{g}^{2}\right)\|\psi\|_{g}^{2}+\frac{\alpha}{4}\left\langle\nabla_{g} f, \nabla_{g}\|\psi\|_{g}^{2}\right\rangle_{g}\right] d \mathrm{Vol}_{g} .
\end{array}
$$


Then one gets

$$
\begin{aligned}
\frac{|\alpha|}{4}\left|\left\langle\nabla_{g} f, \nabla_{g}\|\psi\|_{g}^{2}\right\rangle_{g}\right| & \leq \frac{|\alpha|}{4}\left(c\left\|\nabla_{g} f\right\|_{g}\|\psi\|_{g} \times 2 c^{-1}\left\|\nabla_{A} \psi\right\|_{g}\right) \\
& \leq \frac{|\alpha| c^{2}}{8}\left\|\nabla_{g} f\right\|_{g}^{2}\|\psi\|_{g}^{2}+\frac{c^{-2}|\alpha|}{2}\left\|\nabla_{A} \psi\right\|_{g}^{2}
\end{aligned}
$$

Therefore,

$$
0 \geq \int_{M}\left[\left(1-\frac{c^{-2}|\alpha|}{2}\right)\left\|\nabla_{A} \psi\right\|_{g}^{2}+\frac{1}{4}\|\psi\|_{g}^{4}+\frac{2 \beta-c^{2}|\alpha|}{8}\left\|\nabla_{g} f\right\|_{g}^{2}\|\psi\|_{g}^{2}+\frac{1}{4} S c_{\alpha, \beta}\|\psi\|_{g}^{2}\right] d \mathrm{Vol}_{g},
$$

where $c \neq 0$. If $c^{-2}|\alpha| \leq 2, \beta \geq \frac{c^{2}|\alpha|}{2}$ and $S c_{\alpha, \beta}>0$, then $\psi=0$.

So the conditions $\alpha \in \mathbb{R}, \beta \geq \frac{|\alpha|^{2}}{4}$, and $S c_{\alpha, \beta}>0$ imply $\psi=0$ and then the SW-invariants are zero by the definition of the SW-invariants.

On the other hand, it was shown that when we assume $\alpha \in \mathbb{R}, \beta \geq \frac{|\alpha|^{2}}{6}$, and $S c_{\alpha, \beta}>0$ for the weighted Riemannian 4-manifold $\left(M, g, e^{-f} d \mathrm{vol}_{g}\right)$, then there is a metric $\tilde{g}$ conformal to $g$ with positive scalar curvature in Proposition 4.7. Then, the classical result of Witten also implies that SW-invariants are zero.

The following examples of the weight scalar curvature for weighted Riemannian 4-manifolds $\left(M, g, e^{-f} d \mathrm{vol}_{g}\right)$ are defined in previous literature.

Example 5.3 (Examples of weight scalar curvature).

(1) The positive 4-volumic scalar curvature $S c^{\mathrm{vol}_{4}}(M)>0$ was defined in Definition 3.3 in the Chapter 3.1 and the positive 4-volumic scalar curvature implies

$$
S c_{g}+3 \triangle_{g} f-3\left\|\nabla_{g} f\right\|_{g}^{2}>0
$$

(2) Perelman's scalar curvature [Per02, Section 1]

$$
S c_{g}+2 \triangle_{g} f-\left\|\nabla_{g} f\right\|_{g}^{2}
$$

66 
(3) Chang-Gursky-Yang's conformally invariant scalar curvature [CGY06]:

$$
S c_{g}+\frac{3}{2} \triangle_{g} f-\frac{3}{8}\left\|\nabla_{g} f\right\|_{g}^{2}
$$

Corollary 5.4. Suppose that any one of the 4-volumic scalar curvature, Perelman's scalar curvature, Chang-Gursky-Yang's conformally invariant scalar curvature in a weighted Riemannian 4-manifold $\left(M, g, e^{-f} d \mathrm{vol}_{g}\right)$ is positive, then the Seiberg-Witten invariants of M are zero.

\subsection{General relativity on metric measure spaces}

The Seiberg-Witten invarants provide a connection between scalar curvature and physics in four dimensions. Before that, it is well-known fact that the scalar curvature appears in the Einstein field equations. For this reason, it is not only interesting but also important (in General Relativity) to study the scalar curvature.

Since we have Ricci Bakry-Emery curvature tensor and weighted scalar curvature on the smooth metric measure space, it is natural to try to generalize Relativity Theory to smooth metric measure spaces.

For such a generalization, if one replaces the Ricci and scalar curvature on the left hand side of the Einstein field equations by $\operatorname{Ricc}_{f}^{M}$ and $S c_{\alpha, \beta}$ for the smooth mm-space $\left(M^{n}, g, e^{-f} d \mathrm{Vol}_{g}\right)$, the main meaning question is what is the stress-energy tensor on the right hand side in this case?

We now present several approaches in the literature to answer this question and obtain the appearance of weighted scalar curvature in the Einstein field equations.

The energy condition for Ricci Bakry-Emery curvature tensor is formulated and studied by Woolger [Woo13] and then Rupert-Woolger study the Ricci Bakry-Emery back holes in [RW14]. Hawking's singularity theorem is generalized to the Bakry-Émery framework by Galloway and Woolger in [GW14]. McCann develops the strong energy condition of 
Hawking and Penrose in the (non-smooth) measure metric setting in [McC20]. Mondino and Suhr also find a way to use the optimal transport methods to study the Einstein equations [MS18].

Recently, Shojaie and Rahmanpour propose a conformally invariant theory of gravitation in the context of metric measure space [RS15] [RS16]. If we assume that the cosmological constant $\Lambda$ vanish, then Shojaie-Rahmanpour's field equations are the same as the Einstein field equations.

Inspired by the classical Hilbert-Einstein functional, Fasihi-Ramandi studies the following functional on the space of densities $\mathcal{R}_{f}\left(M^{n}\right):=\{(g, f)\}$ by $\mathcal{L}: \mathcal{R}_{f}\left(M^{n}\right) \rightarrow \mathbb{R}$,

$$
\mathcal{L}(g, f):=\int_{M^{n}} S c_{1,1}(g) d \operatorname{Vol}_{g}=\int_{M^{n}}\left(S c_{g}+\Delta_{g} f-\left\|\nabla_{g} f\right\|_{g}^{2}\right) d \mathrm{Vol}_{g},
$$

and he calls the equations which the critical points of this functional satisfy the new field equations in [FR21]. The new field equations are

$$
\begin{gathered}
R i c_{g}-\frac{1}{2} S c_{g} g=d f \otimes d f-\frac{\left\|\nabla_{g} f\right\|_{g}^{2}}{2} g, \\
\triangle_{g} f=0 .
\end{gathered}
$$

Then Fasihi-Ramandi considers the expression appearing in the right-hand side of the first equation of the new field equations as the stress-energy tensor of matter. It seems his field equations are not the end of story since the equations are completely controlled by the geometry. 


\section{Sphere Theorems with and without Smoothing}

In this chapter, we show two sphere theorems for Riemannian manifolds with scalar curvature bounded below and non-collapsed $\operatorname{RCD}(n-1, n)$ spaces with mean distance close to $\frac{\pi}{2}$.

This chapter is available from Arxiv [Den21].

\subsection{Introduction}

Beginning with the Gauss-Bonnet theorem, sphere theorems show how the geometry can be used to decide the topology. By asking the $\frac{1}{4}$-pinching question (now it is a theorem), Hopf opened the door to the study of sphere theorems. Continuing the paradigm, we will add two kinds of sphere theorems to enrich the subject. We right away state these theorems. Details about the conditions showing up will be given later.

Theorem 6.1. Let $(M, g)$ be an orientable closed Riemannian n-manifold with scalar curvature $\geq n(n-1)$. Suppose there exists a $\left(1, \wedge^{1}\right)$-contracting map $f: M \rightarrow S^{n}$ of nonzero degree and that the map $f$ is harmonic with condition $C \leq 0$, then $f$ is an isometric map.

Remark 6.2. The idea of the proof is based on Chern-Goldberg's argument which was 
used to form the Riemannian version of the Schwarz lemma in [CG75] and their argument originates in S.S. Chern's results generalizing the Schwarz lemma to Hermitian manifolds in [Che68]. In fact, the target of the map can be relaxed to general Einstein manifolds as the proof shows.

Theorem 6.3. Let $\left(X, d, \mathcal{H}^{n}\right)$ be a compact non-collapsed $\operatorname{RCD}(n-1, n)$ space with full support and $\operatorname{md}(X)$ be the mean distance of $\left(X, d, \mathcal{H}^{n}\right)$, then

I. $\operatorname{md}(X) \leq \frac{\pi}{2}$

II. $\operatorname{md}(X)=\frac{\pi}{2}$ if and only if $X$ is isometric to the standard round $n$-sphere $S^{n}$ and $\mathcal{H}^{n}=$ adVol for some a, where $\mathrm{dVol}$ is the volume form of $S^{n}$.

III. there is an $\epsilon(n)>0$ such that $\operatorname{md}(X) \geq \frac{\pi}{2}-\epsilon(n)$ implies that $X$ is homeomorphic to $S^{n}$.

Remark 6.4. Mean distance is an old and well-studied metric invariant in Graph theory, see [DG77]. Note that mean distance is also called distance covariance in Probability, see [Lyo13].

Remark 6.5. We will use Ketterer's maximal diameter theorem [Ket15, Theorem 1.4] and Honda-Mondello's topological sphere theorem [HM19, Theorem A] (for metric measure spaces) to prove Theorem 6.3. We also define a whole family of variants of the concept of mean distance for metric measure spaces and prove sphere theorem for all of them.

\subsection{Harmonic maps with condition C}

Let $S^{n}$ or $\left(S^{n}, g_{s t}\right)$ be the standard round $n$-sphere, then one has a lot of rigidity theorems about $S^{n}$. We will focus on the rigidity theorem with scalar curvature bounded below.

Definition 6.6. Let $M$ and $N$ be two Riemannian manifolds of the same dimension $n$. A smooth map $f: M \rightarrow N$ is said to be $\epsilon$-contracting if $\left\|f_{*} v\right\| \leq \epsilon\|v\|$ for all tangent vectors $v$ on $M$. 
A map $f: M \rightarrow N$ is said to be $\left(\epsilon, \wedge^{k}\right)$-contracting if $\left\|f^{*} \varphi\right\| \leq \epsilon\|\varphi\|$, for all $k$-forms $\varphi \in \wedge^{k}(N)$ and $1 \leq k \leq n$.

Notice that 1-contracting and volume contracting with contraction constant 1 means $\left(1, \wedge^{1}\right)$ - and $\left(1, \wedge^{n}\right)$-contracting, respectively. If $f$ is $\left(1, \wedge^{p}\right)$-contracting, then $f$ is $\left(1, \wedge^{p+k}\right)$ contracting for $k \geq 1$.

Theorem 6.7 (Llarull [Lla95]). For all $n, k, D$ with $n \geq k \geq 3$ and $D>0$ there exists a Riemannian n-manifold $M^{n}$ with scalar curvature $\geq D$ and $a\left(1, \wedge^{k}\right)$-contracting map $f: M^{n} \rightarrow S^{n}$ of degree 1 .

In particular, a volume decreasing non-zero degree map for $M^{n}$ (with scalar curvature bounded below) into $S^{n}$ will in general not be an isometric map .

Confirming and generalizing Gromov's conjecture [Gro86], Llarull showed the following rigidity theorem by index theoretical method.

Theorem 6.8 (Llarull [Lla98]). Let $\left(M^{n}, g\right)$ be a closed, connected, Riemannian spin $n$-manifold with scalar curvature $\geq n(n-1)$. Assume there exists a 1-contracting map or $\left(1, \wedge^{2}\right)$-contracting map $f: M^{n} \rightarrow S^{n}$ of non-zero degree, then $f$ is an isometric map.

Note that the spin condition on $M^{n}$ can be relaxed to require that the map $f$ is a spin map and $S^{n}$ can be replaced by a Riemannian manifold having non-negative curvature operator and satisfying a certain harmonic spinor condition [GS02, Lemma 1.1]. Llarull's rigidity theorem also can be generalized to the weighted rigidity theorem 4.20 on metric measure spaces with positive weighted scalar curvature. The spin condition can not be removed in those two approaches, since the tools of the proofs are the index theory.

Here we will use harmonic maps to approach the rigidity problem about scalar curvature. Furthermore, our argument can be applied to the rigidity question for arbitrary Einstein manifolds. 
We follow the setup and notations in [GI76]. Let $M$ and $N$ be Riemannian $n$-manifolds and $d s_{M}^{2}$ and $d s_{N}^{2}$ be the Riemannian metrics of $M$ and $N$, respectively. Then we can write, locally,

$$
d s_{M}^{2}=\omega_{1}^{2}+\cdots+\omega_{n}^{2}, \quad d s_{N}^{2}=\omega_{1}^{* 2}+\cdots+\omega_{n}^{* 2},
$$

where $\omega_{i}(1 \leq i \leq n)$ and $\omega_{a}^{*}(1 \leq a \leq n)$ are differential 1-forms in $M$ and $N$, respectively. The structure equations in $M$ are

$$
\begin{aligned}
& d \omega_{i}=\sum_{j} \omega_{j} \wedge \omega_{j i} \\
& d \omega_{i j}=\sum_{k} \omega_{i k} \wedge \omega_{k j}-\frac{1}{2} \sum_{k, l} R_{i j k l} \omega_{k} \wedge \omega_{l} .
\end{aligned}
$$

The Ricci tensor $R_{i j}$ is defined by

$$
R_{i j}=\sum_{k} R_{i k j k}
$$

and the scalar curvature by

$$
R=\sum_{i} R_{i i}
$$

Similar equations are valid in $N$ and the corresponding quantities will be denoted in the same notation with asterisks.

Let $f$ be a $C^{\infty}$-mapping of $M$ into $N$ and

$$
f^{*} \omega_{a}^{*}=\sum_{i} A_{i}^{a} \omega_{i}
$$

In the sequel, we will drop $f^{*}$ from such formulas when its presence is clear from the context.

Let $e_{i}$ (resp. $e_{a}^{*}$ ) be the frame dual to the coframe $\omega_{i}$ (resp. $\left.\omega_{a}^{*}\right)$, then we have

$$
f_{*} e_{i}=\sum_{a} A_{i}^{a} e_{a}^{*}
$$

The covariant differential of $A_{i}^{a}$ is defined by

$$
D A_{i}^{a}:=d A_{i}^{a}+\sum_{j} A_{j}^{a} \omega_{j i}+\sum_{b} A_{i}^{b} \omega_{b a}^{*}=: \sum_{j} A_{i j}^{a} \omega_{j}
$$


satisfying

$$
A_{i j}^{a}=A_{j i}^{a}
$$

The following geometrical interpretation of $A_{i j}^{a}$ was given by Chern-Goldberg [CG75, P.136]: Let $x \in M$ and let $T_{x}$ and $T_{f(x)}$ be the tangent spaces at $x$ and $f(x)$, respectively. The mapping

$$
f_{* *}: T_{x} \rightarrow T_{f(x)}
$$

defined by

$$
f_{* *}(v)=\sum_{a, i, j} A_{i j}^{a} \lambda_{i} \lambda_{j} e_{a}^{*}, \quad v=\sum_{i} \lambda_{i} e_{i}
$$

is quadratic and has the property that if $v$ is a unit vector, $f_{* *}(v)$ is the acceleration vector of $f(\gamma)$ at $f(x)$, where $\gamma$ is the geodesic tangent to $v$ at $x$.

Definition 6.9 (Harmonic maps). The mapping $f$ is called harmonic if

$$
\sum_{i} A_{i i}^{a}=0
$$

Notice that the tensor field with the components $\sum_{i} A_{i i}^{a}$ is the tension vector field of Eells-Sampson [ES64] and it was proved by them that the mapping $f$ is harmonic (in Eells-Sampson's sense) if and only if $\sum_{i} A_{i i}^{a}=0$.

Differentiating the equation (6.1) and using the structure equations in $M$ and $N$, we get

$$
\sum_{j} D A_{i j}^{a} \wedge \omega_{j}=-\frac{1}{2} \sum_{j, k, l} A_{l}^{a} R_{j i k l} \omega_{k} \wedge \omega_{l}-\frac{1}{2} \sum_{b, c, d} A_{i}^{b} R_{b a c d}^{*} \omega_{c}^{*} \wedge \omega_{d}^{*}
$$

where

$$
D A_{i j}^{a}:=d A_{i j}^{a}+\sum_{b} A_{i j}^{b} \omega_{b a}^{*}+\sum_{k} A_{k j}^{a} \omega_{k i}+\sum_{k} A_{i k}^{a} \omega_{k j}=: \sum_{k} A_{i j k}^{a} \omega_{k}
$$

Thus, we have

$$
A_{i j k}^{a}-A_{i k j}^{a}=-\sum_{l} A_{l}^{a} R_{l i k j}-\sum_{b, c, d} A_{i}^{b} A_{k}^{c} A_{j}^{d} R_{b a c d}^{*}
$$


By (6.2) and (6.4), we can calculate the Laplacian

$$
\Delta A_{i}^{a}:=\sum_{k} A_{i k k}^{a}=\sum_{k} A_{k i k}^{a}=\sum_{k} A_{k k i}^{a}+\sum_{l} A_{l}^{a} R_{l i}-\sum_{b, c, d, k} R_{b a c d}^{*} A_{k}^{b} A_{k}^{c} A_{i}^{d} .
$$

The ration

$$
A:=\frac{f^{*} d v_{N}}{d v_{M}}
$$

of volume elements has the expression $A=\operatorname{det}\left(A_{i}^{a}\right)$. Let $\left(B_{a}^{i}\right)$ be the adjoint of $\left(A_{j}^{a}\right)$, i.e., $\sum_{a} B_{a}^{i} A_{j}^{a}=\delta_{j}^{i} A$. Then, by 6.1,

$$
d A=\sum_{i, a} B_{a}^{i} d A_{i}^{a}=\sum_{i, j, a} B_{a}^{i} d A_{i j}^{a} \omega_{j}=: \sum_{j} \mathbb{A}_{j} \omega_{j}
$$

Let $V=A^{2}$, then we will compute the Laplacian of $V$ and use it to prove Theorem 6.1 . First, let

$$
d V=\sum_{k} V_{k} \omega_{k}
$$

where

$$
V_{k}=2 A \sum_{i, a} B_{a}^{i} A_{i k}^{a} .
$$

Second, exterior differentiation of 6.6 gives

$$
\sum_{k}\left(d V_{k}-\sum_{i} V_{i} \omega_{k i}\right) \wedge \omega_{k}=0 .
$$

Hence we can write

$$
d V_{k}-\sum_{i} V_{i} \omega_{k i}=\sum_{j} V_{k j} \omega_{j}
$$

where

$$
V_{j k}=V_{k j}
$$

Then the Laplacian of $V$ is by definition equal to

$$
\Delta V=\sum_{k} V_{k k}
$$


Third, by differentiating (6.7), using (6.8), and simplifying, we get

$$
\frac{1}{2} V_{k j}=2 \mathbb{A}_{j} \mathbb{A}_{k}-\sum_{i, l, a, b} B_{a}^{i} B_{b}^{l} A_{l j}^{a} A_{i k}^{b}+A \sum_{i, a} B_{a}^{i} A_{i k j}^{a}
$$

Using (6.5), the Laplacian of $V$ can be expressed as

$$
\frac{1}{2} \Delta V=2 \sum_{j}\left(\mathbb{A}_{j}\right)^{2}+V\left(R-\sum_{b, c, j} R_{b, c}^{*} A_{j}^{b} A_{j}^{c}\right)-C+A \sum_{a, i, j} B_{a}^{i} A_{j j i}^{a},
$$

where $C$ is a scalar invariant of the mapping given by

$$
C=\sum_{\substack{a, b \\ i, j, k}} B_{a}^{i} B_{b}^{k} A_{k j}^{a} A_{i j}^{b}
$$

If $f$ is harmonic, then the last term of 6.9 vanishes. That is, see [CG75, p.141, formula (57)]

(for a harmonic map)

$$
\frac{1}{2} \Delta V=2 \sum_{j}\left(\mathbb{A}_{j}\right)^{2}+V\left(R-\sum_{b, c, j} R_{b, c}^{*} A_{j}^{b} A_{j}^{c}\right)-C .
$$

The geometric meaning of Condition $\mathrm{C}$ 6.10) is given by Chern-Goldberg [CG75, p.142, Remark] as follows: the scalar C may be interpreted geometrically as a weighted measure of the deviation of the square length of the tensor $C_{i j k}$ from the square length of its symmetric part, where $C_{i j k}$ is the pullback of $A_{i j}^{a}$ under $f$.

Proof of Theorem 6.1. From the fact that $S^{n}$ is Einstein, we get

$$
\sum_{b, c, j} R_{b, c}^{*} A_{j}^{b} A_{j}^{c}=\frac{R^{*}}{n} \sum_{a, i}\left(A_{i}^{a}\right)^{2}
$$

Since $M$ is compact and $f$ is of non-zero degree, $V$ does attain its maximum at a point $x$ in $M$. Then $V(x)>0$ and $\Delta V(x) \leq 0$. Notice that $V(x)$ is independent of the choice of the frame and coframe. At the point $x$, we choose a local $g$-orthonormal frame $e_{1}, \ldots, e_{n}$ on $T_{x} M$ and a local $g_{s t}$-orthonormal frame $e_{1}^{*}, \ldots, e_{n}^{*}$ on $T_{f(x)} S^{n}$, such that there exists 
$\lambda_{1} \geq \lambda_{2} \geq \cdots \geq \lambda_{n}>0$, with $f_{*} e_{i}=\lambda_{i} e_{i}^{*}$. This can be done by diagonalizing $f^{*} g_{s t}$ with respect to the metric $g$. As $f$ is $\left(1, \wedge^{1}\right)$-contracting, we have $\lambda_{i} \leq 1$ for all $1 \leq i \leq n$. That means

$$
\sum_{b, c, j} R_{b, c}^{*} A_{j}^{b} A_{j}^{c}=\frac{R^{*}}{n} \sum_{a, i}\left(A_{i}^{a}\right)^{2} \leq R^{*} .
$$

On account of $R^{*}=n(n-1), R \geq n(n-1)$, and $C \leq 0$, we have

$$
\frac{1}{2} \Delta V(x) \geq V(x)(R-n(n-1)) \geq 0 .
$$

Then combining $V(x)>0$ and $\Delta V(x) \leq 0$, we get $R=n(n-1)$ and $\lambda_{i}=1$ for all $1 \leq i \leq n$. That means $f$ is an isometric map.

In fact, the target of the map can be relaxed to an arbitrary Einstein manifold as 6.12) shows.

Corollary 6.10. Let $(N, g)$ be an orientable closed (Riemannian) Einstein n-manifold with scalar curvature $R^{*}$ and $(M, g)$ be an orientable closed Riemannian n-manifold with scalar curvature $R$.

Suppose $R \geq R^{*}$ and there exists a $\left(1, \wedge^{1}\right)$-contracting map $f: M \rightarrow N$ of non-zero degree and the map $f$ is harmonic with condition $C \leq 0$, then $f$ is a locally isometric map. If the fundamental group of $N$ is trivial, then $f$ is an isometric map.

Proof. As $N$ is an Einstein manifold, the inequality 6.12) and $R \geq R^{*}$ implies $f$ is a locally isometric map.

Remark 6.11. It is not clear how to generalize Theorem 6.1 to smooth metric measure spaces with weighted scalar curvature $\geq n(n-1)$.

\subsection{Non-collapsed RCD spaces and mean distance}

The framework of Riemannian manifolds with Ricci curvature bounded below is generalized to metric measure spaces $(X, d, \mu)$ satisfying a Riemannian curvature-dimension 76 
condition and these metric measure spaces are called $\operatorname{RCD}(\kappa, n)$ spaces.

Definition 6.12 (Non-collapsed $\operatorname{RCD}(\kappa, n)$ space). Let $\kappa \in \mathbb{R}, n \in \mathbb{N}$ and $n \geq 1$. The metric measure space $(X, d, \mu)$ is called a non-collapsed $\operatorname{RCD}(\kappa, \mathrm{n})$ space, if

1. $(X, d, \mu)$ is an $\operatorname{RCD}(\kappa, n)$ space.

2. $\mu=\mathcal{H}^{n}$, where $\mathcal{H}^{n}$ is the $n$-dimensional Hausdorff measure with respect to the metric $d$.

The concept of non-collapsed $\operatorname{RCD}(K, N)$ space was defined by De Philippis and Gigli [DPG18, Definition 1.1]. In fact, De Philippis-Gigli showed that $N$ must be an integer. The proof of our theorem below is mainly based on the De Philippis and Gigli's results.

Definition 6.13 (Mean distance). Let $(X, d, \mu)$ be a compact metric measure space with full support, then the mean distance of $X$ is defined as

$$
\operatorname{md}(X):=\int_{X \times X} \frac{d(\cdot, \cdot)}{\mu(X) \times \mu(X)} \mu \otimes \mu
$$

Using Cheng's maximal diameter theorem and Cheeger-Colding's topological sphere theorem [CC97], Kokkendorff [Kok08, Theorem 4] showed following theorem.

Theorem 6.14 (Kokkendorff). Let $\left(M, g, d \mathrm{Vol}_{g}\right)$ be a Riemannian $n$-manifold $(n \geq 2)$ with Ricci curvature $\geq n-1$, where $d \mathrm{Vol}_{g}$ is the Riemannian volume, then

1. $\operatorname{md}(M) \leq \frac{\pi}{2}$.

2. $\operatorname{md}(M)=\frac{\pi}{2}$ if and only if $M$ is isometric to the standard round $n$-sphere $\left(S^{n}, d_{S^{n}}\right)$.

3. there is an $\epsilon^{\prime}(n)>0$ such that $\operatorname{md}(M) \geq \frac{\pi}{2}-\epsilon^{\prime}(n)$ implies that $M$ is diffeomorphic to $S^{n}$

We generalize Kokkendorff's theorem to compact non-collapsed $\operatorname{RCD}(n-1, n)$ spaces.

The strategy of the proof of Theorem 6.3 is the same as Kokkendorff's proof, but we need to replace Cheng's maximal diameter theorem and Cheeger-Colding's topological 
sphere theorem (for the Riemannian manifold) by Ketterer's maximal diameter theorem [Ket15, Theorem 1.4] and Honda-Mondello's topological sphere theorem [HM19. Theorem A] (for metric measure spaces).

The radius $\operatorname{rad}(X, d)$ of a metric space $(X, d)$ is defined by

$$
\operatorname{rad}(X, d):=\inf _{x \in X} \sup _{y \in X} d(x, y)
$$

Before giving the proof of Theorem 6.3, let us collect the relevant properties of a non-collapsed $\operatorname{RCD}(n-1, n)$ space with full support so that we can use them in the proof.

(1). The diameter $\operatorname{diam}(X, d)$ is at most $\pi$ (in particular $\operatorname{rad}(X, d) \leq \pi)[\operatorname{Ket} 15$, Theorem 4.8].

(2). $\left(X, d, \mathcal{H}^{n}\right)$ satisfies the generalized Bishop-Gromov inequality:

$$
\frac{\mathcal{H}^{n}\left(B_{r}(x)\right)}{\mathcal{H}^{n}\left(B_{R}(x)\right)} \geq \frac{\operatorname{Vol}_{S^{n}}\left(B_{r}\right)}{\operatorname{Vol}_{S^{n}}\left(B_{R}\right)}
$$

for any $x \in X$ and $0 \leq r \leq R \pi$, where $B_{r}(x)$ is the closed $r$-ball with center $x$ in $X, B_{s}$ is a closed $s$-ball in the sphere $S^{n}$ and Vol is the volume on the $S^{n}$. Equivalently

$$
\frac{\mathcal{H}^{n}\left(B_{r}(x)\right)}{\mathcal{H}^{n}\left(B_{R}(x)\right)} \geq \frac{\int_{0}^{r}[\sin (t)]^{n-1} d t}{\int_{0}^{R}[\sin (t)]^{n-1} d t}
$$

for any $x \in X$ and $0 \leq r \leq R \leq \pi[$ [DPG18].

(3). [Ketterer's maximal diameter theorem ] If $\operatorname{rad}(X, d)=\pi$, then $(X, d)$ is isometric to $\left(S^{n}, d_{S^{n}}\right)[$ Ket15, Theorem 1.4].

(4). [Honda-Mondello's topological sphere theorem] For all $n \in \mathbb{N}_{\geq 2}$ there exists a positive constant $\epsilon_{1}(n)>0$ such that if a compact metric space $(X, d)$ satisfies that $\operatorname{rad}(X, d) \geq$ $\pi-\epsilon_{1}(n)$, and that $(X, d, \mu)$ is an $\operatorname{RCD}(n-1, n)$ space for some Borel measure $\mu$ on $X$ with full support, then $X$ is homeomorphic to the $n$-dimensional sphere [HM19. Theorem A]. 
Proof of Theorem 6.3 Since the diameter of a non-collapsed $\operatorname{RCD}(n-1, n)$ space is at most $\pi$, we can apply the argument in the proof of Theorem 1.3 in [ES21] to prove I.

Let $\mathrm{D}(x):=\sup \{d(x, y): y \in X\} \leq \pi$ for a fixed $x \in X, h(y):=d(x, y)$, and $\mu_{0}:=\frac{\mathcal{H}^{n}}{\mathcal{H}^{n}(X)}$, then we have

$$
\begin{aligned}
\operatorname{md}(x) & :=\int_{X} h(y) \mu_{0}(d y)=\int_{0}^{\infty} \mu_{0}(\{h \geq s\}) d s \\
& =\int_{0}^{\mathrm{D}(x)}\left[1-\mu_{0}\left(h^{-1}([0, s))\right] d s=\int_{0}^{\mathrm{D}(x)}\left[1-\mu_{0}\left(B_{s}(x)\right)\right] d s,\right.
\end{aligned}
$$

where $B_{s}(x)$ is the closed $s$-ball with center $x$ in $X$. We have the generalized BishopGromov inequality for $\operatorname{RCD}(n-1, n)$ space, i.e.,

$$
\mu_{0}\left(B_{s}(x)\right) \geq \frac{\operatorname{Vol}\left(B_{s}\right)}{\operatorname{Vol}\left(S^{n}\right)}
$$

for $x \in X$ and $0 \leq s \leq \pi$. Hence, we get

$$
\operatorname{md}(x)=\int_{X} h(y) \mu_{0}(d y) \leq \pi-\int_{0}^{\pi} \frac{\operatorname{Vol}\left(B_{s}\right)}{\operatorname{Vol}\left(S^{n}\right)} d s .
$$

As $\operatorname{md}\left(S^{n}\right)=\frac{\pi}{2}$ is shown by Kokkendorff above, we have $\operatorname{md}(x) \leq \frac{\pi}{2}$. That means $\operatorname{md}(X) \leq \frac{\pi}{2}$.

If we have equality $\operatorname{md}(X)=\frac{\pi}{2}$, then the Bishop-Gromov inequality (6.13) must be equality for all $0 \leq s \leq \pi$. It implies that for $\mu_{0}$-a.e. point $x$ there must exists a point $x^{\prime}$ with $d\left(x, x^{\prime}\right)=\pi$. And this must hold even for every $x \in X$, because $X$ is compact and $\mu_{0}$ has full support. Therefore, $\operatorname{rad}(X)=\pi$ and then $(X, d)$ is isometric to $\left(S^{n}, d_{S^{n}}\right)$ by Ketterer's maximal diameter theorem.

We will prove Theorem 6.3 by showing that $\operatorname{md}(X)$ close to $\frac{\pi}{2}$ implies that $\operatorname{rad}(X, d)$ is closed to $\pi$ and then applying Honda-Mondello's topological sphere theorem.

We will prove the claim by contradiction. Suppose that there exists $x_{0} \in X$ such that $D\left(x_{0}\right)<\pi-\epsilon_{1}(n)$ and the $x_{0}$ realizes $\operatorname{rad}(X, d)$. Here we take $\epsilon_{1}(n)$ from HondaMondello's topological sphere theorem. Then $\mu_{0}\left(B_{s}\left(x_{0}\right)\right)$ will achieve its maximum value 1 
for $s<\pi-\epsilon_{1}(n)$. Hence, we get the estimate

$$
\operatorname{md}\left(x_{0}\right)<\frac{\pi}{2}-\int_{\pi-\epsilon_{1}(n)}^{\pi}\left[1-\frac{\operatorname{Vol}\left(B_{s}\right)}{\operatorname{Vol}\left(S^{n}\right)}\right] d s:=\frac{\pi}{2}-\delta\left(\epsilon_{1}(n), n\right) .
$$

Since D is 1-Lipschitz, we have

$$
\mathrm{D}(y)<\pi-\frac{\epsilon_{1}(n)}{2}
$$

for any $y \in B_{\frac{\epsilon_{1}(n)}{2}}\left(x_{0}\right)$. This implies that we have the estimate

$$
\operatorname{md}(y)<\frac{\pi}{2}-\delta\left(\frac{\epsilon_{1}(n)}{2}, n\right)
$$

for any $y \in B_{\frac{\epsilon_{1}(n)}{2}}\left(x_{0}\right)$. Finally, we have

$$
\begin{aligned}
\operatorname{md}(X) & =\int_{X} \operatorname{md}(x) \mu_{0}(d x)=\int_{X \backslash B_{\frac{\epsilon_{1}(n)}{2}}\left(x_{0}\right)} \operatorname{md}(x) \mu_{0}(d x)+\int_{B_{\frac{\epsilon_{1}(n)}{2}}\left(x_{0}\right)} \operatorname{md}(x) \mu_{0}(d x) \\
& <\frac{\pi}{2}-\mu_{0}\left(B_{\frac{\epsilon_{1}(n)}{2}}\left(x_{0}\right)\right) \delta\left(\frac{\epsilon_{1}(n)}{2}, n\right) \leq \frac{\pi}{2}-\frac{\operatorname{Vol}\left(B_{\frac{\epsilon_{1}(n)}{2}}\right)}{\operatorname{Vol}\left(S^{n}\right)} \delta\left(\frac{\epsilon_{1}(n)}{2}, n\right) .
\end{aligned}
$$

Now we take

$$
\epsilon(n)=\frac{\operatorname{Vol}\left(B_{\frac{\epsilon_{1}(n)}{2}}\right)}{\operatorname{Vol}\left(S^{n}\right)} \delta\left(\frac{\epsilon_{1}(n)}{2}, n\right)>0 .
$$

Then $\operatorname{md}(X) \geq \frac{\pi}{2}-\epsilon(n)$ implies $\operatorname{rad}(X, d) \geq \pi-\epsilon_{1}(n)$. Otherwise, it would be a contraction. Then $X$ is homeomorphic to the $n$-dimensional sphere by Honda-Mondello's topological sphere theorem.

Inspired by the concept of mean distance, one can define similar metric invariants. For a compact metric measure space $(X, d, \mu)$ with full support, given a continuous function $\alpha:[0, \infty) \rightarrow \mathbb{R}$, we define the metric invariant $\mathrm{M}_{\alpha}(X)$ by

$$
\mathrm{M}_{\alpha}(X):=\int_{X \times X} \frac{\alpha(d(\cdot, \cdot))}{\mu(X) \times \mu(X)} \mu \otimes \mu
$$

And then one can try to generalize Theorem 6.3 to the $\mathrm{M}_{\alpha}(X)$ case. 
In particular, Erbar and Sturm [ES21] defined

$$
\begin{aligned}
\mathrm{M}_{\mathrm{f}}(X) & :=\int_{X \times X} \frac{f(d(\cdot, \cdot))}{\mu(X) \times \mu(X)} \mu \otimes \mu, \\
\mathrm{M}_{\mathrm{f}, \mathrm{n}}^{*} & :=\frac{\int_{0}^{\pi} f(r)[\sin (r)]^{n-1} d r}{\int_{0}^{\pi}[\sin (r)]^{n-1} d r},
\end{aligned}
$$

where $f:[0, \pi] \rightarrow \mathbb{R}$ is a continuous and strictly increasing function.

Theorem 6.15 (Erbar-Sturm). Let $(X, d, \mu)$ be a compact non-collapsed $\operatorname{RCD}(n-1, n)$ space with $n \geq 1$, then $\mathrm{M}_{\mathrm{f}}(X) \leq \mathrm{M}_{\mathrm{f}, \mathrm{n}}^{*}$ and $\mathrm{M}_{\mathrm{f}}(X)=\mathrm{M}_{\mathrm{f}, \mathrm{n}}^{*}$ if and only if $X$ is isometric to the standard round $n$-sphere $S^{n}$.

Erbar-Sturm also noticed that an analogous statement (with $\mathrm{M}_{\mathrm{f}}(X) \geq \mathrm{M}_{\mathrm{f}, \mathrm{n}}^{*}$ in place of $\mathrm{M}_{\mathrm{f}}(X) \leq \mathbf{M}_{\mathrm{f}, \mathrm{n}}^{*}$ ) holds for strictly decreasing $f$. Without loss of generality, let us assume that $f$ is continuous and strictly increasing. Then we have the following corollary by combining Erbar-Sturm's Theorem and the proof of Theorem 6.3 .

Corollary 6.16. Let $\left(X, d, \mathcal{H}^{n}\right)$ be a compact non-collapsed $\operatorname{RCD}(n-1, n)$ space with full support, then there is an $\epsilon_{2}(f, n)>0$ such that $\mathrm{M}_{\mathrm{f}}(X) \geq \mathrm{M}_{\mathrm{f}, \mathrm{n}}^{*}-\epsilon_{2}(f, n)$ implies that $X$ is homeomorphic to $S^{n}$.

Proof. We only need to show that $\mathrm{M}_{\mathrm{f}}(X)$ close to $\mathrm{M}_{\mathrm{f}, \mathrm{n}}^{*}$ implies that $\operatorname{rad}(X, d)$ is closed to $\pi$. We will prove the claim by contradiction.

Suppose that there exists $x_{0} \in X$ such that $D\left(x_{0}\right)<\pi-\epsilon_{1}(n)$ and the $x_{0}$ realizes $\operatorname{rad}(X, d)$. Here we take $\epsilon_{1}(n)$ from Honda-Mondello's topological sphere theorem. Let $g(y):=f\left(d_{X}\left(x_{0}, y\right)\right), \mu_{0}:=\frac{\mathcal{H}^{n}}{\mathcal{H}^{n}(X)}$, and $\mathrm{M}_{\mathrm{f}}\left(x_{0}\right):=\int_{X} g(y) \mu_{0}(d y)$, then $\mu_{0}\left(B_{s}\left(x_{0}\right)\right)$ will achieve its maximum value 1 for $s<\pi-\epsilon_{1}(n)$.

Hence, we get the estimate

$$
\mathbf{M}_{\mathrm{f}}\left(x_{0}\right)<\mathbf{M}_{\mathrm{f}, \mathrm{n}}^{*}-\int_{f\left(\pi-\epsilon_{1}(n)\right)}^{f(\pi)}\left[1-\frac{\int_{0}^{f^{-1}(s)}[\sin (t)]^{n-1} d t}{\int_{0}^{\pi}[\sin (t)]^{n-1} d t}\right] d s:=\mathbf{M}_{\mathrm{f}, \mathrm{n}}^{*}-\delta_{1}\left(f, \epsilon_{1}(n), n\right) .
$$


Since $\mathrm{D}$ is 1-Lipschitz and $f$ is continuous and strictly increasing, we have

$$
f(\mathrm{D}(y))<f\left(\pi-\frac{\epsilon_{1}(n)}{2}\right)
$$

for any $y \in B_{\frac{\epsilon_{1}(n)}{2}}\left(x_{0}\right)$. This implies that we have the estimate

$$
\left.\mathrm{M}_{\mathrm{f}}(y)<\mathrm{M}_{\mathrm{f}, \mathrm{n}}^{*}-\delta_{1}\left(f, \frac{\epsilon_{1}(n)}{2}\right), n\right)
$$

for any $y \in B_{\frac{\epsilon_{1}(n)}{2}}\left(x_{0}\right)$. Finally, we have

$$
\begin{aligned}
\mathrm{M}_{\mathrm{f}}(X) & =\int_{X} \mathrm{M}_{\mathrm{f}}(x) \mu_{0}(d x)=\int_{X \backslash B_{\frac{\epsilon_{1}(n)}{2}}\left(x_{0}\right)} \mathbf{M}_{\mathrm{f}}(x) \mu_{0}(d x)+\int_{B_{\frac{\epsilon_{1}(n)}{2}}\left(x_{0}\right)} \mathbf{M}_{\mathrm{f}}(x) \mu_{0}(d x) \\
& <\mathbf{M}_{\mathrm{f}, \mathrm{n}}^{*}-\mu_{0}\left(B_{\frac{\epsilon_{1}(n)}{2}}\left(x_{0}\right)\right) \delta_{1}\left(f, \frac{\epsilon_{1}(n)}{2}, n\right) \leq \mathbf{M}_{\mathrm{f}, \mathrm{n}}^{*}-\frac{\operatorname{Vol}\left(B_{\frac{\epsilon_{1}(n)}{2}}\right)}{\operatorname{Vol}\left(S^{n}\right)} \delta_{1}\left(f, \frac{\epsilon_{1}(n)}{2}, n\right) .
\end{aligned}
$$

Now we take

$$
\left.\epsilon_{2}(f, n)=\frac{\operatorname{Vol}\left(B_{\frac{\epsilon_{1}(n)}{2}}\right)}{\operatorname{Vol}\left(S^{n}\right)} \delta_{1}\left(f, \frac{\epsilon_{1}(n)}{2}, n\right)\right)>0 .
$$

Then $\mathrm{M}_{\mathrm{f}}(X) \geq \mathrm{M}_{\mathrm{f}, \mathrm{n}}^{*}-\epsilon(n)$ implies $\operatorname{rad}(X, d) \geq \pi-\epsilon_{1}(n)$. Otherwise, it would be a contraction. Then $X$ is homeomorphic to the $n$-dimensional sphere by Honda-Mondello's topological sphere theorem.

Remark 6.17. The result of Corollary 6.16 is even new (for $f \neq \mathrm{id}$ ) for a Riemannian $n$-manifold with Ricci curvature bounded below by $n(n-1)$. 


\section{Bibliography}

[AC17] Farhan Abedin and Justin Corvino. "On the $P$-scalar curvature". In: J. Geom. Anal. 27.2 (2017), pp. 1589-1623. IssN: 1050-6926. DoI: 10.1007/s12220016-9732-9 (cit. on pp. 48, 53).

[AGS14] Luigi Ambrosio, Nicola Gigli, and Giuseppe Savaré. "Metric measure spaces with Riemannian Ricci curvature bounded from below". In: Duke Math. J. 163.7 (2014), pp. 1405-1490. IssN: 0012-7094. Dor: 10 . 1215/001270942681605 (cit. on pp. 5, 39).

[ADG97] F. D. Ancel, M. W. Davis, and C. R. Guilbault. "CAT(0) reflection manifolds". In: Geometric topology (Athens, GA, 1993). Vol. 2. AMS/IP Stud. Adv. Math. Amer. Math. Soc., Providence, RI, 1997, pp. 441-445 (cit. on p. 14).

[AF94] C. S. Aravinda and F. T. Farrell. "Rank 1 aspherical manifolds which do not support any nonpositively curved metric”. In: Comm. Anal. Geom. 2.1 (1994), pp. 65-78. ISSN: 1019-8385. Dor: 10.4310/CAG . 1994 .v2 .n1 . a4 (cit. on p. 14).

[ALW16] Siva Athreya, Wolfgang Löhr, and Anita Winter. "The gap between Gromovvague and Gromov-Hausdorff-vague topology". In: Stochastic Process. Appl. 126.9 (2016), pp. 2527-2553. IssN: 0304-4149. Dor: 10.1016/j . spa. 2016. 02.009 (cit. on p. 45). 
[BE85] D. Bakry and Michel Émery. "Diffusions hypercontractives". In: Séminaire de probabilités, XIX, 1983/84. Vol. 1123. Lecture Notes in Math. Springer, Berlin, 1985, pp. 177-206. Dor: 10.1007/BFb0075847 (cit. on p. 6).

[BGS85] Werner Ballmann, Mikhael Gromov, and Viktor Schroeder. Manifolds of nonpositive curvature. Vol. 61. Progress in Mathematics. Birkhäuser Boston, Inc., Boston, MA, 1985, pp. vi+263. ISBN: 0-8176-3181-X. DoI: 10.1007/9781-4684-9159-3 (cit. on p.20).

[Bin53] R. H. Bing. "A convex metric with unique segments". In: Proc. Amer. Math. Soc. 4 (1953), pp. 167-174. IssN: 0002-9939. Dor: 10.2307/2032215 (cit. on p. 16.

[Bra97] Hubert Lewis Bray. The Penrose inequality in general relativity and volume comparison theorems involving scalar curvature. Thesis (Ph.D.)-Stanford University. ProQuest LLC, Ann Arbor, MI, 1997, p. 103. ISBN: 978-059160594-5 (cit. on p. 61).

[Bre12] Simon Brendle. "Rigidity phenomena involving scalar curvature". In: Surveys in differential geometry. Vol. XVII. Vol. 17. Surv. Differ. Geom. Int. Press, Boston, MA, 2012, pp. 179-202. DoI: 10 . 4310/SDG . 2012 .v17 .n1 . a4 (cit. on p.61).

[BBI01] Dmitri Burago, Yuri Burago, and Sergei Ivanov. A course in metric geometry. Vol. 33. Graduate Studies in Mathematics. American Mathematical Society, Providence, RI, 2001, pp. xiv+415. ISBN: 0-8218-2129-6. Dor: 10.1090/gsm/ 033 (cit. on pp. 23, 30).

[Bur15] Annegret Y. Burtscher. "Length structures on manifolds with continuous Riemannian metrics”. In: New York J. Math. 21 (2015), pp. 273-296 (cit. on p. 16. 
[Cas13] Jeffrey S. Case. "Conformal invariants measuring the best constants for GagliardoNirenberg-Sobolev inequalities". In: Calc. Var. Partial Differential Equations 48.3-4 (2013), pp. 507-526. ISSN: 0944-2669. Dor: 10 . 1007/s00526-0120559-3 (cit. on p.48).

[CS21] Simone Cecchini and Thomas Schick. "Enlargeable metrics on nonspin manifolds". In: Proc. Amer. Math. Soc. 149.5 (2021), pp. 2199-2211. Issn: 00029939. DoI: $10.1090 / \mathrm{proc} / 14706$ (cit. on pp. 2, 12, 13).

[CGY06] Sun-Yung A. Chang, Matthew J. Gursky, and Paul Yang. "Conformal invariants associated to a measure”. In: Proc. Natl. Acad. Sci. USA 103.8 (2006), pp. 2535-2540. ISSN: 0027-8424. DoI: 10.1073/pnas.0510814103 (cit. on pp. 48, 67).

[CC97] Jeff Cheeger and Tobias H. Colding. "On the structure of spaces with Ricci curvature bounded below. I". In: J. Differential Geom. 46.3 (1997), pp. 406480. IssN: 0022-040X (cit. on pp. 4,77).

[CDS15] Xiuxiong Chen, Simon Donaldson, and Song Sun. "Kähler-Einstein metrics on Fano manifolds. I: Approximation of metrics with cone singularities". In: $J$. Amer. Math. Soc. 28.1 (2015), pp. 183-197. Issn: 0894-0347. Dor: 10.1090/ S0894-0347-2014-00799-2 (cit. on p. 4).

[CMZ15] Xu Cheng, Tito Mejia, and Detang Zhou. "Stability and compactness for complete $f$-minimal surfaces”. In: Trans. Amer. Math. Soc. 367.6 (2015), pp. 4041-4059. ISSN: 0002-9947. Dor: 10.1090/S0002-9947-2015-062072 (cit. on pp. 55, 56).

[Che68] Shiing-shen Chern. "On holomorphic mappings of hermitian manifolds of the same dimension". In: Entire Functions and Related Parts of Analysis (Proc. Sympos. Pure Math., La Jolla, Calif., 1966). Amer. Math. Soc., Providence, R.I., 1968, pp. 157-170 (cit. on p. 70). 
[CG75] Shiing Shen Chern and Samuel I. Goldberg. "On the volume decreasing property of a class of real harmonic mappings". In: Amer. J. Math. 97 (1975), pp. 133-147. IssN: 0002-9327. Dor: 10.2307/2373664 (cit. on pp. 8, 70,73, 75).

[CS13] Diarmuid Crowley and Thomas Schick. "The Gromoll filtration, $\mathrm{KO}$-characteristic classes and metrics of positive scalar curvature”. In: Geom. Topol. 17.3 (2013), pp. 1773-1789. ISSN: 1465-3060. Dor: 10.2140/gt.2013.17.1773 (cit. on p. 51.

[DJL12] M. Davis, T. Januszkiewicz, and J.-F. Lafont. "4-dimensional locally CAT(0)manifolds with no Riemannian smoothings". In: Duke Math. J. 161.1 (2012), pp. 1-28. ISSN: 0012-7094. Dor: 10.1215/00127094-1507259 (cit. on pp.4, $13-15)$.

[Dav83] Michael W. Davis. "Groups generated by reflections and aspherical manifolds not covered by Euclidean space”. In: Ann. of Math. (2) 117.2 (1983), pp. $293-$ 324. ISSN: 0003-486X. DoI: 10.2307/2007079 (cit. on p. 14).

[DJ91] Michael W. Davis and Tadeusz Januszkiewicz. "Hyperbolization of polyhedra". In: J. Differential Geom. 34.2 (1991), pp. 347-388. IssN: 0022-040X (cit. on p. 14).

[DPG18] Guido De Philippis and Nicola Gigli. "Non-collapsed spaces with Ricci curvature bounded from below". In: J. Éc. polytech. Math. 5 (2018), pp. 613-650. ISSN: 2429-7100. Dor: 10.5802/jep.80 (cit. on pp. 39, 77, 78).

[Den21a] Jialong Deng. “Curvature-dimension condition meets Gromov's $n$-volumic scalar curvature”. In: SIGMA Symmetry Integrability Geom. Methods Appl. 17 (2021), 013, 20 pages. Dor: 10.3842/SIGMA .2021.013 (cit. on pp. 5,7, 31, 47). 
[Den21b] Jialong Deng. "Enlargeable length-structure and scalar curvatures". In: Ann. Global Anal. Geom. 60.2 (2021), pp. 217-230. Issn: 0232-704X. Dor: 10 1007/s10455-021-09772-7 (cit. on pp. 3, 11).

[Den21] Jialong Deng. "Sphere Theorems with and without Smoothing”. In: arXiveprints, arXiv:2105.13088 (May 2021), arXiv:2105.13088. arXiv: 2105.13088 [math.DG] (cit. on pp. 8, 69,.

[Don96] S. K. Donaldson. "The Seiberg-Witten equations and 4-manifold topology". In: Bull. Amer. Math. Soc. (N.S.) 33.1 (1996), pp. 45-70. Issn: 0273-0979. Dor: 10.1090/S0273-0979-96-00625-8 (cit. on p. 64).

[DG77] J. K. Doyle and J. E. Graver. "Mean distance in a graph". In: Discrete Math. 7.2 (1977), pp. 147-154. IssN: 0012-365X. Dor: 10.1016/0012-365X(77) 90144-3 (cit. on pp. 8, 70).

[ES64] James Eells Jr. and J. H. Sampson. "Harmonic mappings of Riemannian manifolds". In: Amer. J. Math. 86 (1964), pp. 109-160. IssN: 0002-9327. Dor: 10.2307/2373037 (cit. on p.73).

[ES21] Matthias Erbar and Karl-Theodor Sturm. "Rigidity of cones with bounded Ricci curvature". In: J. Eur. Math. Soc. (JEMS) 23.1 (2021), pp. 219-235. IssN: 1435-9855. Dor: $10.4171 / \mathrm{jems} / 1010$ (cit. on pp. 79, 81).

[Fan08] Edward M. Fan. “Topology of three-manifolds with positive $P$-scalar curvature". In: Proc. Amer. Math. Soc. 136.9 (2008), pp. 3255-3261. IssN: 00029939. Dor: 10.1090/S0002-9939-08-09066-7 (cit. on p. 57).

[FJ93] F. T. Farrell and L. E. Jones. "Topological rigidity for compact non-positively curved manifolds". In: Differential geometry: Riemannian geometry (Los Angeles, CA, 1990). Vol. 54. Proc. Sympos. Pure Math. Amer. Math. Soc., Providence, RI, 1993, pp. 229-274 (cit. on p. 14). 
[FJ91] F. Thomas Farrell and Lowell E. Jones. "Rigidity in geometry and topology". In: Proceedings of the International Congress of Mathematicians, Vol. I, II (Kyoto, 1990). Math. Soc. Japan, Tokyo, 1991, pp. 653-663 (cit. on p.14).

[FR21] Ghodratallah Fasihi-Ramandi. “The Bakry-Émery Ricci Tensor: Application to Mass Distribution in Space-time”. In: Gravit. Cosmol. 27.1 (2021), pp. 4246. ISSN: 0202-2893. Dor: 10.1134/S0202289321010096 (cit. on p. 68).

[Fed69] Herbert Federer. Geometric measure theory. Die Grundlehren der mathematischen Wissenschaften, Band 153. Springer-Verlag New York Inc., New York, 1969, pp. xiv+676 (cit. on p. 29).

[FCS80] Doris Fischer-Colbrie and Richard Schoen. "The structure of complete stable minimal surfaces in 3-manifolds of nonnegative scalar curvature”. In: Comm. Pure Appl. Math. 33.2 (1980), pp. 199-211. IssN: 0010-3640. Dor: 10.1002/ cpa.3160330206 (cit. on p. 57).

[GW14] Gregory J. Galloway and Eric Woolgar. "Cosmological singularities in BakryÉmery spacetimes". In: J. Geom. Phys. 86 (2014), pp. 359-369. Issn: 03930440. Dor: $10.1016 / \mathrm{j}$.geomphys .2014.08.016 (cit. on p. 67).

[Gig15] Nicola Gigli. "On the differential structure of metric measure spaces and applications”. In: Mem. Amer. Math. Soc. 236.1113 (2015), pp. vi+91. IssN: 0065-9266. Dor: 10.1090/memo/1113 (cit. on pp. 6. 45).

[GS02] S. Goette and U. Semmelmann. "Scalar curvature estimates for compact symmetric spaces”. In: Differential Geom. Appl. 16.1 (2002), pp. 65-78. IssN: 0926-2245. DoI: 10.1016/S0926-2245(01)00068-7 (cit. on pp. 61, 71).

[GI76] Samuel I. Goldberg and Tôru Ishihara. "Harmonic quasiconformal mappings of Riemannian manifolds". In: Amer. J. Math. 98.1 (1976), pp. 225-240. IssN: 0002-9327. Dor: 10.2307/2373623 (cit. on p. 72). 
[GV79] A. Gray and L. Vanhecke. "Riemannian geometry as determined by the volumes of small geodesic balls". In: Acta Math. 142.3-4 (1979), pp. 157-198. ISSN: 0001-5962. DOI: 10.1007/BF02395060 (cit. on p. 36).

[Gro86] M. Gromov. "Large Riemannian manifolds". In: Curvature and topology of Riemannian manifolds (Katata, 1985). Vol. 1201. Lecture Notes in Math. Springer, Berlin, 1986, pp. 108-121. Dor: 10 . 1007/BFb0075649 (cit. on p.71.

[Gro96] M. Gromov. "Positive curvature, macroscopic dimension, spectral gaps and higher signatures". In: Functional analysis on the eve of the 21st century, Vol. II (New Brunswick, NJ, 1993). Vol. 132. Progr. Math. Birkhäuser Boston, Boston, MA, 1996, pp. 1-213. Dor: 10.1007/s10107-010-0354-x (cit. on p.61.

[Gro03] M. Gromov. "Isoperimetry of waists and concentration of maps". In: Geom. Funct. Anal. 13.1 (2003), pp. 178-215. Issn: 1016-443X. Dor: 10 . $1007 /$ s000390300004 (cit. on p. 55).

[GL80] Mikhael Gromov and H. Blaine Lawson Jr. "Spin and scalar curvature in the presence of a fundamental group. I'. In: Ann. of Math. (2) 111.2 (1980), pp. 209-230. IssN: 0003-486X. Dor: 10.2307/1971198 (cit. on pp. 2, 12, 13 , 27).

[GL83] Mikhael Gromov and H. Blaine Lawson Jr. "Positive scalar curvature and the Dirac operator on complete Riemannian manifolds". In: Inst. Hautes Études Sci. Publ. Math. 58 (1983), 83-196 (1984). IssN: 0073-8301 (cit. on pp.2, 12. 54).

[Gro14] Misha Gromov. "Dirac and Plateau billiards in domains with corners". In: Cent. Eur. J. Math. 12.8 (2014), pp. 1109-1156. IssN: 1895-1074. Dor: 10 2478/s11533-013-0399-1 (cit. on p. 57). 
[Gro17] Misha Gromov. 101 Questions, Problems and Conjectures around Scalar Curvature. https : //www . ihes . fr/ gromov/wp-content/uploads / 2018/08/101-problems0ct1-2017 .pdf. 2017 (cit. on pp. 5, 22, 31, 32, 61).

[Gro18] Misha Gromov. "Metric inequalities with scalar curvature". In: Geom. Funct. Anal. 28.3 (2018), pp. 645-726. IssN: 1016-443X. Dor: 10. 1007/s00039018-0453-z (cit. on pp. 12, 19, 40, 53).

[Gro19] Misha Gromov. "Four Lectures on Scalar Curvature". In: arXiv e-prints, arXiv:1908.10612 (Aug. 2019), arXiv:1908.10612. arXiv: 1908.10612 [math.DG] (cit. on pp. 2, 13, 21, 61).

[HS06] B. Hanke and T. Schick. "Enlargeability and index theory". In: J. Differential Geom. 74.2 (2006), pp. 293-320. IssN: 0022-040X (cit. on p. 27).

[HPS15] Bernhard Hanke, Daniel Pape, and Thomas Schick. "Codimension two index obstructions to positive scalar curvature". In: Ann. Inst. Fourier (Grenoble) 65.6 (2015), pp. 2681-2710. IssN: 0373-0956 (cit. on p. 50).

[HH58] Friedrich Hirzebruch and Heinz Hopf. "Felder von Flächenelementen in 4dimensionalen Mannigfaltigkeiten”. In: Math. Ann. 136 (1958), pp. 156-172. ISSN: 0025-5831. DOI: 10.1007/BF01362296 (cit. on p. 64).

[Hit74] Nigel Hitchin. "Harmonic spinors". In: Advances in Math. 14 (1974), pp. 1-55. ISSN: 0001-8708. DOI: 10.1016/0001-8708(74)90021-8 (cit. on pp.2,22, 51).

[HM19] Shouhei Honda and Ilaria Mondello. "Sphere theorems for RCD and stratified spaces". In: arXiv e-prints, arXiv:1907.03482 (July 2019), arXiv:1907.03482. arXiv: 1907.03482 [math.DG] (cit. on pp. 8, 70, 78). 
[KS03] Gennadi Kasparov and Georges Skandalis. "Groups acting properly on "bolic" spaces and the Novikov conjecture”. In: Ann. of Math. (2) 158.1 (2003), pp. 165-206. IssN: 0003-486X. Dor: 10.4007/annals.2003.158.165 (cit. on p. 13.

[Kaz82] Jerry L. Kazdan. "Deformation to positive scalar curvature on complete manifolds". In: Math. Ann. 261.2 (1982), pp. 227-234. IssN: 0025-5831. DoI: 10.1007/BF01456220 (cit. on p. 33).

[KW75] Jerry L. Kazdan and F. W. Warner. "Scalar curvature and conformal deformation of Riemannian structure”. In: J. Differential Geometry 10 (1975), pp. 113134. IssN: 0022-040X (cit. on pp. 1, 22, 33).

[Ket15] Christian Ketterer. "Cones over metric measure spaces and the maximal diameter theorem”. In: J. Math. Pures Appl. (9) 103.5 (2015), pp. 1228-1275. IssN: 0021-7824. Dor: 10.1016/j . matpur. 2014 . 10.011 (cit. on pp. 8, 70, 78).

[Kok08] Simon L. Kokkendorff. "Characterizing the round sphere by mean distance". In: Differential Geom. Appl. 26.6 (2008), pp. 638-644. IssN: 0926-2245. DoI: $10.1016 / \mathrm{j}$. difgeo.2008.04.012 (cit. on p.77).

[Lan19] Christian Lange. "When is the underlying space of an orbifold a manifold?" In: Trans. Amer. Math. Soc. 372.4 (2019), pp. 2799-2828. Issn: 0002-9947. Dor: $10.1090 / \operatorname{tran} / 7687$ (cit. on p. 15).

[Las97] Jean B. Lasserre. "On the setwise convergence of sequences of measures". In: J. Appl. Math. Stochastic Anal. 10.2 (1997), pp. 131-136. Issn: 1048-9533. DOI: $10.1155 /$ S1048953397000166 (cit. on p. 42).

[LY72] H. Blaine Lawson Jr. and Shing Tung Yau. "Compact manifolds of nonpositive curvature". In: J. Differential Geometry 7 (1972), pp. 211-228. Issn: 0022040X (cit. on p. 14). 
[LS14] Dan A. Lee and Christina Sormani. "Stability of the positive mass theorem for rotationally symmetric Riemannian manifolds”. In: J. Reine Angew. Math. 686 (2014), pp. 187-220. IssN: 0075-4102. DoI: 10.1515/crelle-2012-0094 (cit. on p.6).

[LP87] John M. Lee and Thomas H. Parker. “The Yamabe problem”. In: Bull. Amer. Math. Soc. (N.S.) 17.1 (1987), pp. 37-91. IssN: 0273-0979. DoI: 10. 1090/ S0273-0979-1987-15514-5 (cit. on p. 51).

[Lic63] André Lichnerowicz. "Spineurs harmoniques". In: C. R. Acad. Sci. Paris 257 (1963), pp. 7-9. Issn: 0001-4036 (cit. on p.2).

[Lla95] Marcelo Llarull. "Scalar curvature and volume of a Riemannian manifold". In: Geom. Dedicata 56.1 (1995), pp. 1-4. IssN: 0046-5755. Dor: 10.1007/ BF01263608 (cit. on p.71).

[Lla98] Marcelo Llarull. "Sharp estimates and the Dirac operator". In: Math. Ann. 310.1 (1998), pp. 55-71. IssN: 0025-5831. DoI: 10 . 1007 /s002080050136 (cit. on pp. 19, 58, 71).

[Loh18] Joachim Lohkamp. "Contracting Maps and Scalar Curvature”. In: arXiv eprints, arXiv:1812.11839 (2018), arXiv:1812.11839. arXiv: 1812.11839 [math.DG] (cit. on p. 19).

[LV09] John Lott and Cédric Villani. "Ricci curvature for metric-measure spaces via optimal transport”. In: Ann. of Math. (2) 169.3 (2009), pp. 903-991. IssN: 0003-486X. Dor: 10.4007/annals.2009.169.903 (cit. on pp. 4, 31).

[LP20] Danka Lučić and Enrico Pasqualetto. "Infinitesimal Hilbertianity of weighted Riemannian manifolds". In: Canad. Math. Bull. 63.1 (2020), pp. 118-140. ISSN: 0008-4395. DOI: 10.4153/s0008439519000328 (cit. on p. 54). 
[Lyo13] Russell Lyons. "Distance covariance in metric spaces". In: Ann. Probab. 41.5 (2013), pp. 3284-3305. IssN: 0091-1798. Dor: 10.1214/12-AOP803 (cit. on pp. 8, 70).

[McC20] Robert J. McCann. "Displacement convexity of Boltzmann's entropy characterizes the strong energy condition from general relativity". In: Camb. J. Math. 8.3 (2020), pp. 609-681. ISSN: 2168-0930. Dor: 10.4310/CJM.2020.v8.n3.a4 (cit. on p.68).

[MS18] Andrea Mondino and Stefan Suhr. "An optimal transport formulation of the Einstein equations of general relativity". In: arXiv e-prints, arXiv:1810.13309 (Oct. 2018), arXiv:1810.13309. arXiv: 1810 . 13309 [math-ph] (cit. on p. 68.

[Oht07] Shin-ichi Ohta. "On the measure contraction property of metric measure spaces”. In: Comment. Math. Helv. 82.4 (2007), pp. 805-828. Issn: 0010-2571. Dor: $10.4171 / \mathrm{CMH} / 110$ (cit. on pp. 5, 39).

[Oht09] Shin-ichi Ohta. "Finsler interpolation inequalities". In: Calc. Var. Partial Differential Equations 36.2 (2009), pp. 211-249. IssN: 0944-2669. Dor: 10. 1007/s00526-009-0227-4 (cit. on p. 45).

[Osa20] Damian Osajda. "Small cancellation labellings of some infinite graphs and applications". In: Acta Math. 225.1 (2020), pp. 159-191. IssN: 0001-5962. Dor: 10.4310/acta.2020.v225.n1.a3 (cit. on p. 15).

[Per02] Grisha Perelman. "The entropy formula for the Ricci flow and its geometric applications”. In: arXiv Mathematics e-prints, math/0211159 (Nov. 2002), math/0211159. arXiv: math/0211159 [math.DG] (cit. on pp. 4, 47, 66).

[Pet11] Anton Petrunin. “Alexandrov meets Lott-Villani-Sturm”. In: Münster J. Math. 4 (2011), pp. 53-64. Isss: 1867-5778 (cit. on pp. 5, 32). 
[Qia97] Zhongmin Qian. "Estimates for weighted volumes and applications". In: Quart. J. Math. Oxford Ser. (2) 48.190 (1997), pp. 235-242. IssN: 0033-5606. Dor: 10.1093/qmath/48.2.235 (cit. on pp. 47,53).

[RS15] Nafiseh Rahmanpour and Hossein Shojaie. "A conformally invariant theory of gravitation in metric measure space”. In: Gen. Relativity Gravitation 47.10 (2015), Art. 123, 16. IssN: 0001-7701. DoI: 10.1007/s10714-015-1962-9 (cit. on p.68).

[RS16] Nafiseh Rahmanpour and Hossein Shojaie. "Metric measure space as a framework for gravitation”. In: Gen. Relativity Gravitation 48.10 (2016), Art. 122, 16. ISSN: 0001-7701. Dor: 10.1007/s10714-016-2123-5 (cit. on p. 68).

[Ros+08] César Rosales et al. "On the isoperimetric problem in Euclidean space with density". In: Calc. Var. Partial Differential Equations 31.1 (2008), pp. 27-46. ISSN: 0944-2669. DOI: 10.1007/s00526-007-0104-y (cit. on p. 55).

[Ros83] Jonathan Rosenberg. " $C^{*}$-algebras, positive scalar curvature, and the Novikov conjecture". In: Inst. Hautes Études Sci. Publ. Math. 58 (1983), 197-212 (1984). ISSN: 0073-8301 (cit. on pp. 2, 50).

[Ros07] Jonathan Rosenberg. "Manifolds of positive scalar curvature: a progress report”. In: Surveys in differential geometry. Vol. XI. Vol. 11. Surv. Differ. Geom. Int. Press, Somerville, MA, 2007, pp. 259-294. Dor: 10.4310/SDG . 2006 v11.n1.a9 (cit. on p. 2).

[RW14] M. Rupert and E. Woolgar. "Bakry-Émery black holes”. In: Classical Quantum Gravity 31.2 (2014), pp. 025008, 16. IssN: 0264-9381. Dor: 10.1088/02649381/31/2/025008 (cit. on p. 67). 
[Sap14] Mark Sapir. “A Higman embedding preserving asphericity”. In: J. Amer. Math. Soc. 27.1 (2014), pp. 1-42. IssN: 0894-0347. Dor: 10 . 1090/S0894-03472013-00776-6 (cit. on p. 15).

[Sch14] Thomas Schick. "The topology of positive scalar curvature". In: Proceedings of the International Congress of Mathematicians-Seoul 2014. Vol. II. Kyung Moon Sa, Seoul, 2014, pp. 1285-1307 (cit. on p. 2).

[SY79a] R. Schoen and S. T. Yau. "On the structure of manifolds with positive scalar curvature". In: Manuscripta Math. 28.1-3 (1979), pp. 159-183. IssN: 00252611. Dor: 10.1007/BF01647970 (cit. on p. 1).

[SY79b] R. Schoen and Shing Tung Yau. "Existence of incompressible minimal surfaces and the topology of three-dimensional manifolds with nonnegative scalar curvature”. In: Ann. of Math. (2) 110.1 (1979), pp. 127-142. Issn: 0003-486X. Dor: $10.2307 / 1971247$ (cit. on pp. 2, 55, 57).

[Sch84] Richard Schoen. "Conformal deformation of a Riemannian metric to constant scalar curvature". In: J. Differential Geom. 20.2 (1984), pp. 479-495. IssN: 0022-040X (cit. on p. 52).

[SY17] Richard Schoen and Shing-Tung Yau. "Positive Scalar Curvature and Minimal Hypersurface Singularities”. In: arXiv e-prints, arXiv:1704.05490 (2017), arXiv:1704.05490. arXiv: 1704.05490 [math.DG](cit. on pp. 2,11,13).

[SS79] L. Siebenmann and D. Sullivan. "On complexes that are Lipschitz manifolds". In: Geometric topology (Proc. Georgia Topology Conf., Athens, Ga., 1977). Academic Press, New York-London, 1979, pp. 503-525 (cit. on p. 15).

[Sto92] Stephan Stolz. "Simply connected manifolds of positive scalar curvature". In: Ann. of Math. (2) 136.3 (1992), pp. 511-540. Issn: 0003-486X. Dor: 10.2307/ 2946598 (cit. on p.2). 
[Sto76] David A. Stone. "Geodesics in piecewise linear manifolds". In: Trans. Amer. Math. Soc. 215 (1976), pp. 1-44. IsSN: 0002-9947. Dor: 10.2307/1999713 (cit. on p. 15).

[Stu06a] Karl-Theodor Sturm. "On the geometry of metric measure spaces. I". In: Acta Math. 196.1 (2006), pp. 65-131. ISSN: 0001-5962. DoI: 10.1007/s11511006-0002-8 (cit. on pp. 4, 31).

[Stu06b] Karl-Theodor Sturm. "On the geometry of metric measure spaces. II". In: Acta Math. 196.1 (2006), pp. 133-177. IssN: 0001-5962. Dor: 10.1007/s11511006-0003-7 (cit. on pp. 33, 37).

[Tau94] Clifford Henry Taubes. "The Seiberg-Witten invariants and symplectic forms". In: Math. Res. Lett. 1.6 (1994), pp. 809-822. IssN: 1073-2780. Dor: 10.4310/ MRL. 1994.v1.n6.a15 (cit. on p. 64).

[Ver18] Giona Veronelli. "Scalar curvature via local extent". In: Anal. Geom. Metr. Spaces 6.1 (2018), pp. 146-164. IssN: 2299-3274. Dor: 10.1515/agms-20180008 (cit. on p. 17).

[Vi109] Cédric Villani. Optimal transport. Vol. 338. Grundlehren der Mathematischen Wissenschaften [Fundamental Principles of Mathematical Sciences]. Old and new. Springer-Verlag, Berlin, 2009, pp. xxii+973. IsBn: 978-3-540-71049-3. Dor: $10.1007 / 978-3-540-71050-9$ (cit. on pp. 31, 45).

[Wit94] Edward Witten. "Monopoles and four-manifolds". In: Math. Res. Lett. 1.6 (1994), pp. 769-796. IsSN: 1073-2780. DoI: 10.4310/MRL . 1994.v1.n6.a13 (cit. on p.64).

[Woo13] Eric Woolgar. "Scalar-tensor gravitation and the Bakry-Émery-Ricci tensor". In: Classical Quantum Gravity 30.8 (2013), pp. 085007, 8. IssN: 0264-9381. Dor: $10.1088 / 0264-9381 / 30 / 8 / 085007$ (cit. on p. 67). 
[Wyl15] William Wylie. "Sectional curvature for Riemannian manifolds with density". In: Geom. Dedicata 178 (2015), pp. 151-169. Issn: 0046-5755. Dor: 10.1007/ s10711-015-0050-3 (cit. on p.47).

[You82] Lai Sang Young. "Dimension, entropy and Lyapunov exponents”. In: Ergodic Theory Dynam. Systems 2.1 (1982), pp. 109-124. IssN: 0143-3857. Dor: 10. $1017 / \mathrm{s} 0143385700009615$ (cit. on p. 33).

[Zei17] Rudolf Zeidler. "An index obstruction to positive scalar curvature on fiber bundles over aspherical manifolds”. In: Algebr. Geom. Topol. 17.5 (2017), pp. 3081-3094. ISSN: 1472-2747. Dor: 10.2140/agt.2017.17.3081 (cit. on p. 50).

[ZZ10] Hui-Chun Zhang and Xi-Ping Zhu. "Ricci curvature on Alexandrov spaces and rigidity theorems”. In: Comm. Anal. Geom. 18.3 (2010), pp. 503-553. IssN: 1019-8385. DoI: 10.4310/CAG.2010.v18.n3.a4 (cit. on pp. 5, 32). 